\title{
Single-order
}

\section{Lamellar Multilayer Gratings}





\section{SiNGLE-ORDER \\ Lamellar Multilayer Gratings}

door

Robert van der Meer 


\section{Ph.D. committee}

\section{Chairman \& secretary:}

Prof. dr. G. van der Steenhoven

\section{Promotor:}

Prof. dr. F. Bijkerk

\section{Co-promotor:}

Prof. dr. K.-J. Boller

\section{Members:}

Prof. dr. A. I. Erko

Prof. dr. H. P. Urbach

Dr. ir. E. M. C. M. Reuvekamp

Prof. dr. ir. W. G. van der Wiel

Prof. dr. ing. A. J. H. M. Rijnders
University of Twente

University of Twente \& FOM Institute DIFFER

University of Twente

Helmholtz Zentrum Berlin Delft University of Technology PANalytical University of Twente University of Twente

Cover: 3D artist impression of a Lamellar Multilayer Grating (LMG). A Transmission Electron Microscope image of an actual LMG is shown on the rear. The grating was etched into a 400 bi-layer W/Si multilayer mirror with a total stack height $H$ of $1 \mu \mathrm{m}$ and a tungsten layer thickness of only 3 atoms. The grating had a period $D$ of $300 \mathrm{~nm}$ and a lamel width $\Gamma D$ of $75 \mathrm{~nm}$. In comparison to the TEM image on the rear, the red and green layers in the artist impression correspond to tungsten and silicon, respectively. The bi-layer period in the artist impression is overestimated by a factor of 10 for visibility.

(C) Robert van der Meer (2013)

Single-order Lamellar Multilayer Gratings

Ph.D. thesis, University of Twente, Enschede, The Netherlands

Illustrated - With references - With summary in English and Dutch

ISBN: 978-90-365-3523-6

DOI: $10.3990 / 1.9789036535236$

Printed by Gildeprint drukkerijen, Enschede, The Netherlands 


\title{
SINGLE-ORDER \\ Lamellar Multilayer Gratings
}

\author{
PROEFsCHRIFT
}

ter verkrijging van

de graad van doctor aan de Universiteit Twente,

op gezag van de rector magnificus,

prof. dr. H. Brinksma,

volgens besluit van het College voor Promoties

in het openbaar te verdedigen

op vrijdag 22 maart 2013 om 12.45 uur

door

Robert van der Meer

geboren op 5 september 1985

te Aberdeen, Schotland 
Dit proefschrift is goedgekeurd door de promotor

Prof. dr. F. Bijkerk

en de co-promotor

Prof. dr. K.-J. Boller

The research presented in this thesis was carried out at the Laser Physics and Nonlinear Optics group, Department of Science and Technology, MESA ${ }^{+}$ Institute of Nanotechnology, University of Twente, P.O. Box 217, 7500 AE Enschede, The Netherlands. This research is supported by the Dutch Technology Foundation STW, which is part of the Netherlands Organisation for Scientific Research (NWO) and partly funded by the Ministry of Economic Affairs (project number \#10302). 


\section{Summary}

A major challenge in the soft x-ray (SXR) and eXtreme UltraViolet (XUV) spectral ranges is the ability to manipulate the incident radiation using optical elements. By patterning conventional multilayer mirrors with nanoscale structures, novel optical elements with a variety of optical properties can be obtained. In this work, the design, fabrication and characterization of Lamellar Multilayer Gratings (LMG) was investigated. Such LMGs are a particular form of the general class of Bragg-Fresnel optics which combine Fresnel optics with Bragg reflection to provide unique dispersive and focusing optics. In particular, LMGs can be used to improve the spectral resolution of x-ray fluorescence techniques.

A Coupled Waves Approach (CWA) was derived to simulate the optical performance, in terms of resolution and reflectivity, of LMGs. This CWA allowed to study the physical principles behind LMGs and resulted in the identification of an optimal LMG operating regime. In this regime, the incident beam is reflected in a single diffraction order and is hence referred to as the singleorder regime. Such single-order LMGs were fabricated using UV-NanoImprint Lithography and Bosch Deep Reactive Ion Etching, a process chosen to enable rapid practical development. Single-order operation was experimentally demonstrated and showed an improvement in spectral resolution of a factor of 3.8 with regard to conventional multilayer mirrors. Singe-order excitation of higher diffraction orders was also measured and analyzed.

Single-order operation often requires multilayer stacks and grating structures that cannot be fabricated with sufficient accuracy using current technologies. As this invalidates the semi-infinite multilayer approximation, we investigated the optical performance of LMGs with finite multilayer stacks. We determined the ratio between the absorber thickness and bi-layer period of the multilayer stack can be used to further tailor LMG optical performance to maximize bandwidth reduction or minimize peak reflectivity loss. We also investigated various degradation processes that could limit the lifetime of LMGs, which is important for the applicability of such elements. Oxidation of tungsten and silicon as well as changes to the sidewall composition were clearly seen. However, SXR reflectivity remained stable to within measurement accuracy for an extended storage period of 18 months in a $1 \mathrm{~atm}$ air environment. The possibility of applying a capping layer on LMG structures to reduce degradation was also studied. 



\section{Samenvatting}

Een grote uitdaging in het zachte röntgen (SXR) en eXtreme UltraViolet (XUV) golflengte gebied is de mogelijkheid om deze straling te manipuleren door middel van optische elementen. Door conventionele multilaag spiegels te structureren kunnen nieuwe optische elementen gemaakt worden met een grote verscheidenheid in het optisch gedrag. In dit werk is het ontwerp, fabricage en karakterisatie van de zogenoemde Lamellaire Multilaag Tralies (LMG) onderzocht. Zulke LMGs zijn een specifieke vorm van Bragg-Fresnel elementen die Fresnel optica combineert met Bragg reflectie om zo unieke dispersieve en focuserende eigenschappen te verkrijgen. In het bijzonder kunnen deze LMGs worden gebruikt om de spectrale resolutie van röntgen fluoresentie technieken te verbeteren.

Een theorie genaamd Gekoppelde Golven Aanpak (CWA) is afgeleid om het optisch gedrag, in termen van resolutie en reflectiviteit, van LMGs te kunnen simuleren. Deze CWA maakt het mogelijk om de optica van LMG elementen te bestuderen. Optimaal LMG gedrag wordt bereikt in het enkelvoudige-orde regime, waarin de inkomende röntgenbundel slechts één diffractie orde exciteert. Deze enkelvoudige-orde LMGs zijn vervolgens gefabriceerd met UVNanoImprint Lithografie en Bosch Deep Reactive Ion Etching. Hiermee is de werking in het enkelvoudige orde voor het eerst experimenteel aangetoond. Dit leidde tot een verbetering in spectrale resolutie met een factor 3.8 in vergelijking met conventionele multilaagspiegels. Het gebruik van hogere orders is ook bestudeerd en geanalyseerd.

Het enkelvoudige-orde regime vergt multilagen en tralie structuren die niet met de huidige technieken voldoende accuraat gemaakt kunnen worden. De semi-oneindige multilaag benadering is daardoor niet altijd geldig en wij hebben daarom het optisch gedrag van LMGs met een eindige multilaag bestudeerd. Uit deze analyse hebben wij bepaald dat de verhouding tussen de dikte van de absorberende component en de bi-laag periode van de multilaag gebruikt kan worden om LMG optisch gedrag verder te optimaliseren voor kleine tralie structuren. Tot slot hebben wij degradatieprocessen onderzocht die de levensduur van LMGs zouden kunnen verminderen. Hoewel er duidelijke veranderingen in de compositie van de zijlaag en oxidatie is waargenomen, bleef de SXR reflectie stabiel binnen de meetnauwkeurigheid gedurende een opslagperiode van 18 maanden in een 1 atm lucht omgeving. Het gebruik van een beschermende afdeklaag op LMGs tegen degradatie is ook onderzocht. 



\section{Publications}

\section{Journal papers}

- I. V. Kozhevnikov, R. van der Meer, H. M. J. Bastiaens, K.-J. Boller and F. Bijkerk, "High-resolution, high-reflectivity operation of lamellar multilayer amplitude gratings: identification of the single-order regime", Optics Express, vol. 18, no. 15, pp. 16234-16242, 2010

- II. V.V. Kozhevnikov, R. van der Meer, H. M. J. Bastiaens, K.-J. Boller and F. Bijkerk, "Analytic theory of soft x-ray diffraction by lamellar multilayer gratings", Optics Express, vol. 19, no. 10, pp. 9172-9184, 2011

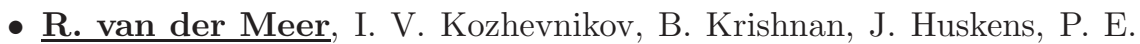
Hegeman, G. C. S. Brons, B. Vratzov, H. M. J. Bastiaens, K.-J. Boller and F. Bijkerk, "Single-order operation of lamellar multilayer gratings in the soft x-ray spectral range", AIP Advances, vol. 3, no. 1, pp. 012103-1/7, 2013

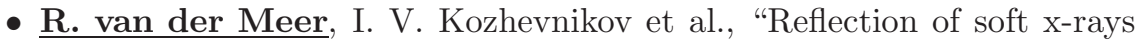
from non-ideal Lamellar Multilayer Gratings", In preparation.

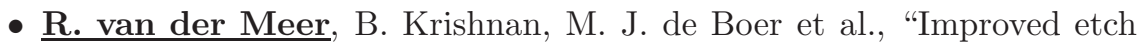
anisotropy in Bosch DRIE using thin-layered mixed materials", In preparation.

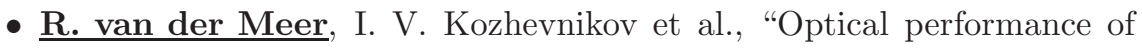
single-order lamellar multilayer gratings with finite multilayer stacks", In preparation.

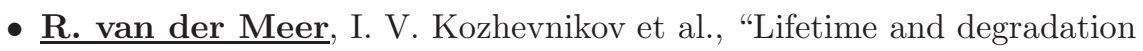
effects in patterned W/Si multilayer mirrors", In preparation.

- V. V. Medvedev, A. J. R. van den Boogaard, R. van der Meer, A. E. Yakshin, E. Louis, V. M. Krivtsun, A. M. Yakunin and F. Bijkerk, "Infrared phase-shift filtering for Extreme UltraViolet multilayer Bragg reflectors", In preparation. 


\section{Patents}

- F. Bijkerk, W. G. van der Wiel, R. van der Meer and P. E. Hegeman, "Method for manufacturing a multilayer structure with a lateral pattern for application in the XUV wavelength range, and BF and LMAG structures manufactured according to this method", International patent WO 2011/071380, Priority date: 11/12/2009, Publication date: 16/06/2011

- F. van Goor, F. Bijkerk, A. J. R. van den Boogaard and R. van der Meer, "Spectral filter for splitting a beam with electromagnetic radiation having wavelengths in the extreme ultraviolet (EUV) or soft x-ray (soft $\mathrm{x}$ ) and the infrared (IR) wavelength range", International patent WO 2012/023853, Priority date: 18/08/2010, Publication date: 23/02/2012

\section{Conference proceedings}

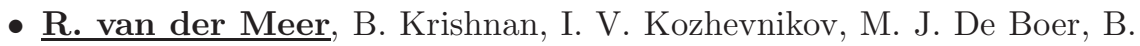
Vratzov, H. M. J. Bastiaens, J. Huskens, W. G. van der Wiel, P. E. Hegeman, G. C. S. Brons, K.-J. Boller and F. Bijkerk, "Improved resolution for soft-x-ray monochromatization using lamellar multilayer gratings", in Advances in X-Ray/EUV Optics and Components VI, vol. 8139 of Proc. of SPIE, pp. 81390Q-1/8, 2011

\section{Oral presentations}

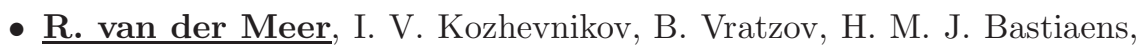
K.-J. Boller and F. Bijkerk, "Lamellar Multilayer Amplitude Gratings for use in XUV optics", EOS topical meeting on Diffractive Optics 2010, 14/02/2010, Koli, Finland

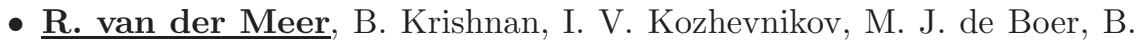
Vratzov, H. M. J. Bastiaens, J. Huskens, W. G. van der Wiel, P. E. Hegeman, G. C. S. Brons, K.-J. Boller and F. Bijkerk, "Improved resolution for soft-x-ray monochromatization using lamellar multilayer gratings", FOM Veldhoven 2011, 17/01/2011, Veldhoven, the Netherlands

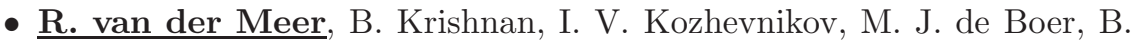
Vratzov, H. M. J. Bastiaens, J. Huskens, W. G. van der Wiel, P. E. Hegeman, G. C. S. Brons, K.-J. Boller and F. Bijkerk, "Improved resolution for soft-x-ray spectroscopy: single-order operation of lamellar multilayer gratings", EOS topical meeting on Diffractive Optics 2012, 27/02/2012, Delft, the Netherlands

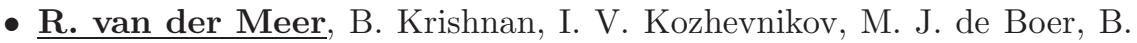
Vratzov, H. M. J. Bastiaens, J. Huskens, W. G. van der Wiel, P. E. 
Hegeman, G. C. S. Brons, K.-J. Boller and F. Bijkerk, "Etching of W/Si multilayer mirrors for the fabrication of lamellar multilayer gratings", Micro and NanoEngineering 2012, 17/09/2012, Toulouse, France

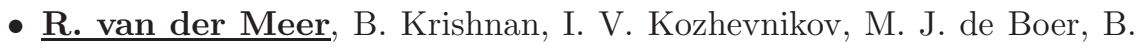
Vratzov, H. M. J. Bastiaens, J. Huskens, G. P. M. Roelofs, P. V. Santos, W. G. van der Wiel, P. E. Hegeman, G. C. S. Brons, K.-J. Boller and F. Bijkerk, "Advantages of UV-NanoImprint Lithography and dualsource dry etching for novel applications", MicroNanoConference 2012, 10/12/2012, Ede, the Netherlands

\section{Poster presentations}

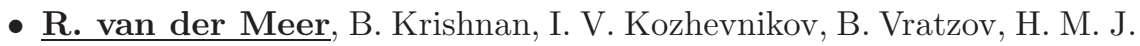
Bastiaens, J.Huskens, W. G. van der Wiel, K.-J. Boller and F. Bijkerk, "Fabrication of lamellar multilayer gratings using UV-NIL and modified Bosch etching", MicroNanoConference, 17-18/10/2010, Enschede, the Netherlands

- P. F. Moonen, B. Krishnan, B. Vratzov, R. van der Meer, K.-J. Boller, W. G. van der Wiel, H. M. J. Bastiaens, W. T. T. Smaal, M. Péter, D. Turkenburg, E. R. Meinders, F. Bijkerk and J. Huskens, "Nanofabrication by Step-and-flash Imprint Lithography", MESA ${ }^{+}$annual meeting, 14/09/2010, Enschede, the Netherlands

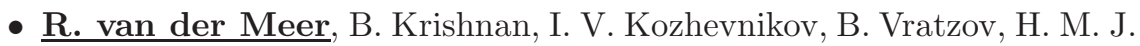
Bastiaens, J. Huskens, W. G. van der Wiel, K.-J. Boller and F. Bijkerk, "Improved resolution for soft-x-ray monochromatization using lamellar multilayer gratings", NNV Plasma physics, 15-16/03/2011, Lunteren, the Netherlands

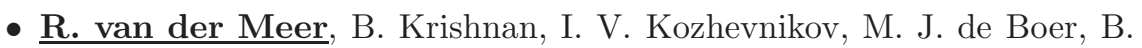
Vratzov, H. M. J. Bastiaens, J. Huskens, W. G. van der Wiel, P. E. Hegeman, G. C. S. Brons, K.-J. Boller and F. Bijkerk, "Improved resolution for soft-x-ray monochromatization using lamellar multilayer gratings", SPIE Optics and Photonics, 21-25/08/2011, San Diego, United States

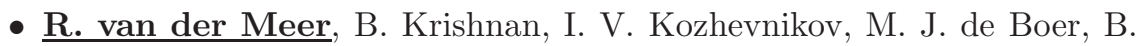
Vratzov, H. M. J. Bastiaens, J. Huskens, W. G. van der Wiel, P. E. Hegeman, G. C. S. Brons, K.-J. Boller and F. Bijkerk, "Improved resolution for soft-x-ray monochromatization using lamellar multilayer gratings", $\mathrm{MESA}^{+}$annual meeting, 27/09/2011, Enschede, the Netherlands

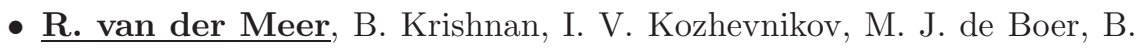
Vratzov, H. M. J. Bastiaens, J.Huskens, W. G. van der Wiel, P. E. Hegeman, G. C. S. Brons, K.-J. Boller and F. Bijkerk, "Improved resolution 
for soft-x-ray monochromatization using lamellar multilayer gratings", MicroNanoConference, 15-16/11/2011, Ede, the Netherlands

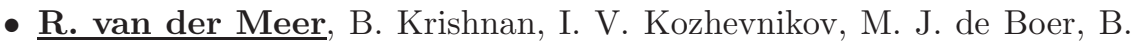
Vratzov, H. M. J. Bastiaens, J.Huskens, W. G. van der Wiel, P. E. Hegeman, G. C. S. Brons, K.-J. Boller and F. Bijkerk, "Reduced bandwidths for soft-x ray reflection using Lamellar Multilayer Gratings", NNV plasma physics, 06-07/03/2012, Lunteren, the Netherlands

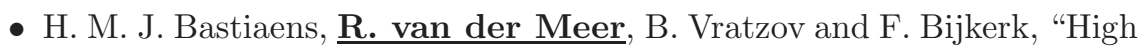
line density free-standing transmission gratings for EUV emission spectroscopy", International Symposium on Extreme Ultraviolet Lithography, 30/09-04/10/2012, Brussels, Belgium 


\section{Contents}

Summary

Samenvatting $\quad$ iii

Publications $\quad$ v

I Journal papers ................... $\mathrm{v}$

II Patents ...................... . . . vi

III Conference proceedings ............... . . vi

IV Oral presentations ................... vi

$\mathrm{V}$ Poster presentations .................... vii

1 Introduction $\quad 1$

1.1 Motivation ..................... 1

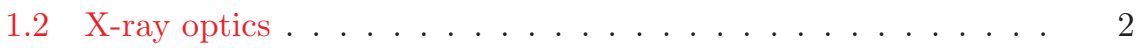

1.2.1 Multilayer mirrors . . . . . . . . . . . . . . 2

1.2 .2 Diffractive optics ................. . . . . 4

1.2.3 Lamellar Multilayer Gratings . . . . . . . . . . . . 7

1.3 Applications...................... 8

1.3.1 X-ray fluorescence spectroscopy . . . . . . . . . 8

1.3.2 X-ray imaging ................. . . . . 9

1.3.3 EUV lithography . . . . . . . . . . . . . 10

1.4 Thesis outline . . . . . . . . . . . . . 10

2 Experimental $\quad \mathbf{1 3}$

2.1 Introduction . . . . . . . . . . . . . . . . . . . 13

2.2 Optical performance and multilayer structure characterization . 13

2.2.1 Soft x-ray reflectometry . . . . . . . . . . 13

2.2.2 Grazing incidence x-ray reflectometry . . . . . . . . . 14

2.3 LMG structure characterization . . . . . . . . . . . . . . . 15

2.3.1 Stylus profilometry . . . . . . . . . . 15

2.3.2 Scanning electron microscopy ... . . . . . . 15 
2.3.3 Transmission electron microscopy . . . . . . . . . . 16

2.3.4 X-ray photoelectron spectroscopy . . . . . . . . 17

3 Theory of Lamellar Multilayer Gratings 19

3.1 Introduction . . . . . . . . . . . . . . . . . 19

3.2 Coupled waves approach . . . . . . . . . . . . . 20

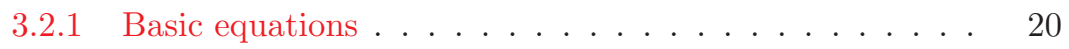

3.2.2 Generalized equations . . . . . . . . . . . 23

3.2.3 Numerical solution . . . . . . . . . . . . . 25

3.2.4 Analytical solution . . . . . . . . . . . 26

3.2.5 Analytic description of LMG optical performance . . . . 30

3.2.6 Interfacial roughness . . . . . . . . . . . . . . . . . 31

3.2.7 CWA validity for p-polarized radiation . . . . . . . . 32

3.3 Single-order LMG operation . . . . . . . . . . . . . . . . . . 34

3.4 Conclusions ..................... 36

$4 \quad$ Fabrication of gratings in W/Si multilayer mirrors $\quad 37$

4.1 Introduction . . . . . . . . . . . . . . 37

4.2 Experimental . . . . . . . . . . . . . . . 38

4.3 Etching and scalloping in Bosch DRIE . . . . . . . . . . . . 39

4.4 Determination of fabrication criteria . . . . . . . . . . . 40

4.4.1 Generalized Coupled Waves Approach . . . . . . . . . . 41

4.4.2 Optical effect of tapering . . . . . . . . . . . 42

4.4.3 Optical effect of scalloping. . . . . . . . . . . 44

4.4.4 Fabrication criteria for W/Si LMGs . . . . . . . . . . 46

4.5 Etching of W/Si ML mirrors . . . . . . . . . . . . . . 47

4.5.1 Etch settings . . . . . . . . . . . 47

4.5.2 Comparison of ML with silicon etching . . . . . . . . . . 48

4.5.3 Control of scallop formation . . . . . . . . . . . 51

4.5.4 Minimizing profile taper ........... 52

4.5.5 Reduction of grating structure dependence . . . . . 53

4.6 Conclusions ......................... 53

5 Experimental demonstration of single-order LMG operation $\mathbf{5 5}$

5.1 Introduction . . . . . . . . . . . . . . 55

5.2 Single versus multi-order operation . . . . . . . . . . . . 55

5.3 Single-order $\Gamma$ scaling . . . . . . . . . . . . . 57

5.4 Bi-layer period accuracy . . . . . . . . . . . . . 58

5.5 Energy dependence of LMG performance . . . . . . . . . . 59 
5.6 Higher diffraction orders . . . . . . . . . . . . . . . 60

5.7 Conclusions . . . . . . . . . . . . . . . 62

6 Performance of finite stack Lamellar Multilayer Gratings $\quad 63$

6.1 Introduction . . . . . . . . . . . . . . 63

6.2 Semi-infinite approximation .............. 64

6.2.1 Reflection from single-order LMGs . . . . . . . . . . . . 64

6.2.2 Analytical description of optical performance . . . . . 65

6.2.3 Simulation of semi-infinite LMGs . . . . . . . . . . . . 67

6.3 Finite ML stacks . . . . . . . . . . . . . . . . . . 67

6.3.1 Simulation of LMGs with finite ML stacks . . . . . . . . 67

6.3.2 Tailoring of performance with the $\gamma$-ratio . . . . . . 69

6.4 Conclusions . . . . . . . . . . . . . . . . . 70

7 Lifetime of patterned W/Si multilayer mirrors $\quad 73$

7.1 Introduction . . . . . . . . . . . . . . . . . 73

7.2 Potential degradation processes . . . . . . . . . . . 74

7.2.1 Sidewall contamination ............ . . 74

7.2.2 Thermal annealing . . . . . . . . . . . . 80

7.2.3 Oxygen diffusion ................. 83

7.2.4 Stability of SXR reflectivity over time . . . . . . . . 84

7.3 Capping layers . . . . . . . . . . . . . . . 85

7.3.1 Possible capping materials . . . . . . . . . 85

7.3.2 Preliminary silicon nitride deposition tests . . . . . . . 86

7.4 Conclusions . . . . . . . . . . . . . . . 88

8 Conclusions $\quad 89$

$\begin{array}{lc}\text { References } & 93\end{array}$

$\begin{array}{ll}\text { Dankwoord/Acknowledgments } & 103\end{array}$ 



\section{1 \\ Introduction}

\subsection{Motivation}

The ability to identify and quantify chemical elements in products is very important to ensure, for instance, that steel and concrete are of sufficient quality to be used for construction and that there are no toxic contaminants in our food or drinks. One of the earliest methods used to identify elements is the flame test in which a sample is positioned in a hot, transparent flame. The heat from the flame breaks chemical bonds to create free atoms and also excites these atoms into excited electronic states. When these atoms return to the ground state, they emit light at characteristic wavelengths. For example, calcium will produce a yellow-red colored flame whereas copper produces a green flame and lead results in a bluish-white flame. However, the range of detectable elements is small and the sample is burned during the measurement [1].

A major improvement in chemical element identification was achieved after the discovery of x-rays. Henry Moseley determined in 1914 that there was a relationship between the atomic number $Z$ of elements and the energy of fluorescent x-rays emitted after an element was irradiated by high energy xrays $[2,3]$. Elements could now be uniquely and non-destructively identified by analyzing the fluorescent x-ray spectrum, which is the basic principle of x-ray fluorescence spectroscopy as described in more detail in section 1.3.1.

To analyze the fluorescence spectrum, an optical element is required that separates x-rays with different energies. Such elements are typically natural crystals or ML mirrors which work based on the well-known principle of Bragg diffraction as discussed in more detail in section 1.2.1. The energy of fluorescent $\mathrm{x}$-rays depend on the difference in binding energies of the electron orbitals for each specific element. Due to their electronic configuration, lighter elements 
can be identified best in the soft x-ray (SXR) spectral range, i.e. at energies between roughly 100 and $1500 \mathrm{eV}$. Unfortunately, the spectral resolution of ML mirrors in this regime is inherently limited by absorption of the fluorescent $\mathrm{x}$-rays that need to be analyzed [4-7]. Although the natural line widths of fluorescent $\mathrm{x}$-rays are typically $E / \Delta E \approx 1000$ [8], closely spaced lines could often not be distinguished due to the limited resolution of the analyzing optical element.

The spectral resolution of ML mirrors can be improved by fabricating a grating into the ML stack, resulting in a so-called Lamellar Multilayer Grating (LMG). Although this concept has long been known [9-12], the implementation of LMGs has been hampered by two main challenges. The first was limitations of previous simulation methods, which provided limited insights in LMG operating principles and could only be used for idealized LMG structures. The second main challenge was the lack of a reproducible LMG fabrication method with accurate dimension control that is suitable for grating periods well below $1 \mu \mathrm{m}$ and can be used to economically pattern large area's (tens of $\mathrm{mm}^{2}$ ).

The usability of LMGs has been significantly improved by the simulation and fabrication method that will be presented in this thesis. We derived a Coupled Waves Analysis (CWA) method which can be used to simulate reflection and diffraction from LMGs and circumvents the aforementioned limitations. This CWA also resulted in improved understanding of LMG working principles and was used to identify an optimal operating regime. In this regime, the incident beam excites only a single diffraction order and is hence referred to as the single-order regime. We also developed a novel fabrication process based on UV-NanoImprint Lithography and Bosch Deep Reactive Ion Etching, which provided reproducible and cost-effective fabrication of single-order operating LMGs with grating periods down to $200 \mathrm{~nm}$. With our LMGs, we achieved spectral resolutions $E / \Delta E$ of up to 330 as compared to previously reported values of 70 [13] for conventional ML mirrors.

Although we focused on LMGs, this study can be considered as a pilot case study for other optical elements that can be fabricated using the process presented in this thesis and that also combine Bragg reflection and diffraction.

\section{$1.2 \quad$ X-ray optics}

LMGs are a specific type of optical element in the general class of Bragg-Fresnel optics in which Bragg reflection by a ML mirror is combined with a diffractive optical element. In this section, we first examine the reflection from ML mirrors and discuss the basic principles of diffraction in some more detail.

\subsubsection{Multilayer mirrors}

A multilayer (ML) mirror consists of a stack of at least two different materials that provide multiple reflecting surfaces. One of the materials is generally 
high- $Z$ (high $\delta$, red layer in figure 1.1) to provide a layer for reflection, whereas the other is a low- $Z$ material (low $\delta$, green layer in figure 1.1) to provide spacing between the high- $Z$ layers. At the interfaces from the low to high- $Z$ material, external reflection occurs. By carefully selecting the layer thickness, all reflected beams add up in phase when the bi-layer period $d$ of each period (the combination of a low and high- $Z$ layer) satisfies the well-known Bragg condition $[14,15]$ :

$$
j \lambda=2 d \sin (\Theta),
$$

in which $j$ is the Bragg order, $\lambda$ is the incident wavelength and $\Theta$ is the grazing incidence angle. Although this Bragg condition is sufficiently accurate for most purposes, it does not consider differences in path length in the ML itself. This is included in the corrected Bragg condition giving higher accuracy [16, 17]:

$$
j \lambda=2 d \sin (\Theta)\left[1-\frac{4 \bar{\delta} d^{2}}{j^{2} \lambda^{2}}\right],
$$

in which $\bar{\delta}$ is a density profile averaged value of $\delta$ for a bi-layer pair.

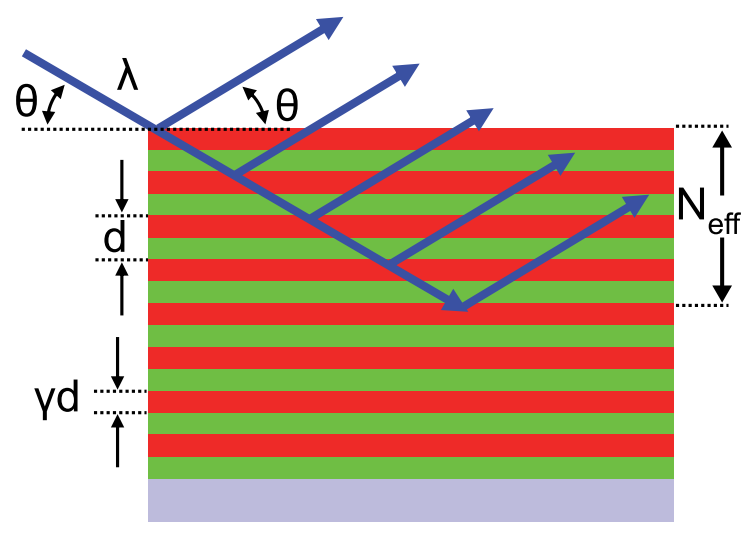

Figure 1.1: An incident beam with wavelength $\lambda$ and incident angle $\Theta$ is reflected at the interfaces of the multilayer mirror. If the angle, wavelength, and bi-layer period $d$ fulfill the Bragg condition (1.1) constructive interference occurs. The penetration depth of the incident beam determines the bandwidth as the bandwidth scales with $1 / N_{\text {eff }}$.

The Bragg condition also reveals that multilayer mirrors are wavelength dispersive elements, i.e. each wavelength is only reflected at a specific angle for every Bragg order. Constructive interference can only occur within a certain bandwidth $\Delta \Theta$ depending on the number of bi-layers $\left(N_{e f f}\right)$ effectively contributing to the reflection. The spectral resolution of a ML mirror, defined 
as $\lambda / \Delta \lambda$, scales with $N_{\text {eff }}[18,19]$. However, absorption in the SXR is actually relatively high and maximum SXR path lengths through materials are in the order of only a few $\mu \mathrm{m}$. This limits $N_{\text {eff }}$, resulting in typical spectral resolutions around 50-100 [13].

Besides the spectral resolution, the peak reflectivity is also of great importance when characterizing a ML mirror to provide as much intensity in the reflected beam as possible. The peak reflectivity of a ML depends on the wavelength, materials and parameters such as the absorber-thickness-tobi-layer-period ratio $\gamma$. As the wavelength is generally known, the choice of ML materials and parameters should be adapted to the wavelength, or wavelength range, of interest.

The choice of materials for a ML mirror is mainly determined by the complex refractive index $(n=1-\delta+i \beta)$, although deposition and stability issues should also be considered. For optimal reflection, a large contrast in $\delta$ between spacer and absorber is required with minimal absorption (low $\beta$ ) in both materials. Suitable material combinations can be selected based on the optical properties using a $\beta$ against $\delta$ plot for the wavelength of interest, as shown for $1.25 \mathrm{~nm}$ in figure 1.2a. From this figure, it can easily be seen that silicon is an excellent candidate as low- $Z$ material. The optimal high- $Z$ material can then be chosen by maximizing the modulation-over-absorption ratio $\Lambda=\operatorname{Re}\left|\epsilon_{A}-\epsilon_{S}\right| / \operatorname{Im}\left(\epsilon_{A}\right)$, where $\epsilon$ is the dielectric constant given by $n=\sqrt{\epsilon}[4]$. This is demonstrated in figure $1.2 \mathrm{~b}$ for the SXR energy of $1 \mathrm{keV}$ and assuming silicon to be the spacer layer. Considering not only the $\Lambda$-ratio, but also deposition and chemical properties of the various elements, tungsten was determined to be the best absorber candidate for the SXR energy around $1 \mathrm{keV}$.

Having characterized the optical performance, in terms of bandwidth and peak reflectivity, of ML mirrors and determined appropriate ML materials, we next discuss the basic principle of diffraction.

\subsubsection{Diffractive optics}

Diffraction is the property of waves to spread, upon propagation, also in the transverse direction. This is most notable when a wave encounters an obstacle, such as opaque or phase shifting materials. An example in every day life is the rainbow pattern often seen on CDs or DVDs, which is caused by their closely spaced tracks acting as a diffraction grating.

Diffraction patterns can be described using the Huygens-Fresnel principle [20-22]. This principle assumes that every disturbance of an incident wave results in point sources emitting secondary spherical waves and that the diffraction pattern can then be determined by examining the interference of these secondary waves. By fabricating a structure with optical disturbances at specific locations, various diffraction patterns can thus be obtained. Two commonly used diffractive structures are gratings and zoneplates [14].

Constructive interference from a grating is achieved for angles at which 


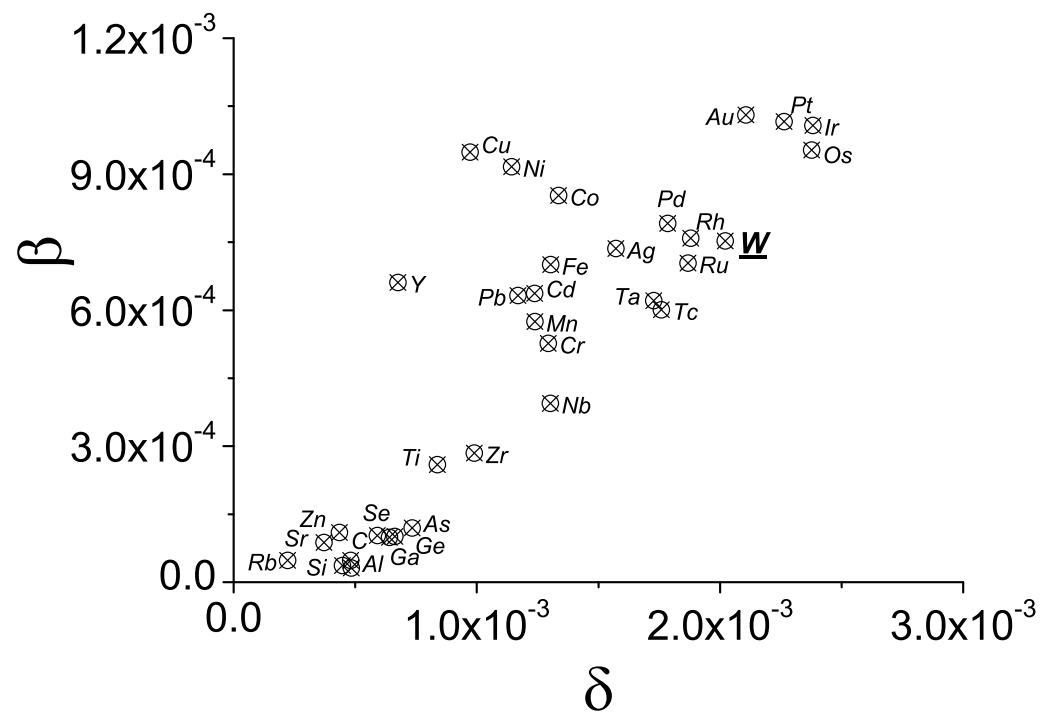

(a) $\beta$ against $\delta$ plot of the complex refractive index $(n=1-\delta+i \beta)$ for various elements.

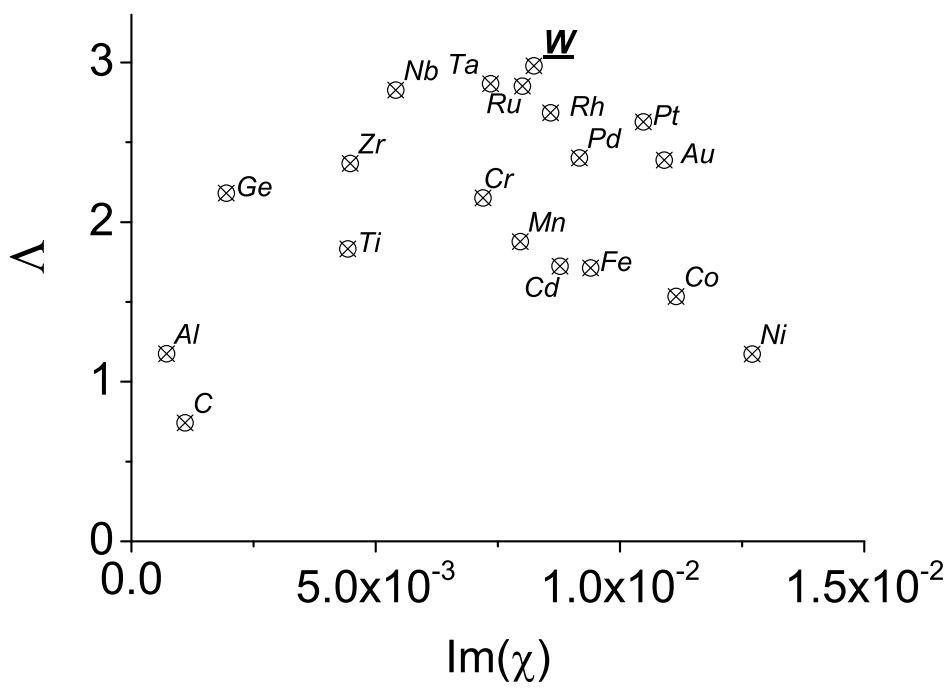

(b) The modulation-over-absorption ratio $\Lambda$ can be used to determine the optimal absorber material. Here, silicon was assumed to be the spacer material and unfavorable elements due to optical, chemical or deposition reasons were removed with respect to figure 1.2a.

Figure 1.2: Absorber material choice at the incident wavelength $\lambda$ of $1.25 \mathrm{~nm}(E=1 \mathrm{keV})$ and assuming silicon to be the spacer material. 
the optical path length difference for waves coming from two neighboring lines equals an integer times the wavelength, leading to the grating equation:

$$
m \lambda=D\left(\cos \Theta_{0}-\cos \Phi_{m}\right),
$$

where $m$ is the diffraction order, $D$ is the grating period and $\Phi$ is the exiting angle of the diffracted beam. For a diffraction order to exist, constructive interference should not only occur between waves emitted from neighboring lines, but also from all waves emitted from within a single line. The variation in irradiance $I$ due to the interference from the single lines is described by Fraunhofer diffraction [14]:

$$
I(\Theta)=I(0) \operatorname{sinc}((k b / 2) \sin \Theta)^{2},
$$

where $k=2 \pi / \lambda$ and $b$ is the line width. From this equation, angles of destructive interference from a single grating line can be determined to occur at [14]:

$$
M \lambda=b \sin \Theta_{M},
$$

where $M$ indicates the diffraction order from a single line. The total diffraction pattern then equals product of the grating and single-slit interference patterns.

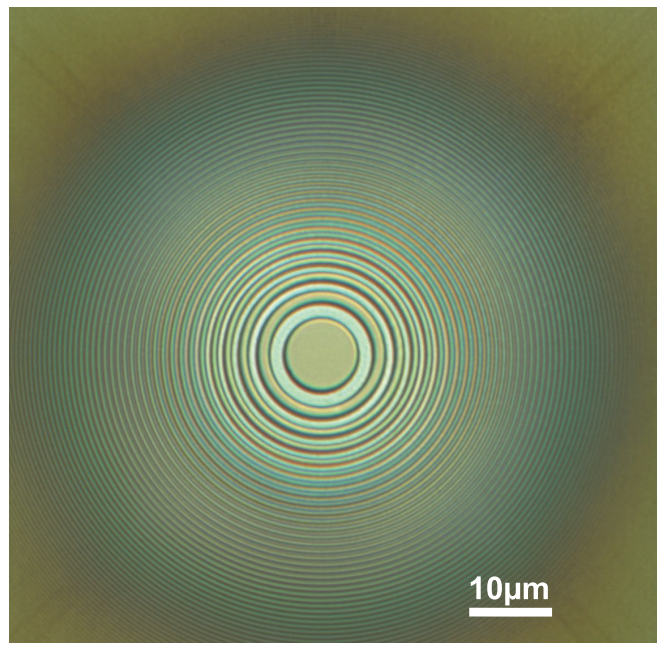

Figure 1.3: Optical image of a zoneplate for the EUV $(13.5 \mathrm{~nm})$ spectral region with a focal length of $18.5 \mathrm{~cm}$ that was imprinted with UV-NIL. The zoneplate has a total diameter of $250 \mu \mathrm{m}$ and consists of 781 zones with a central zone diameter of $9 \mu \mathrm{m}$ and an outer zone width of $80 \mathrm{~nm}$.

A more dedicated diffractive structure is the Fresnel zoneplate. Zoneplates make use of diffraction to focus light, unlike lenses which are based on refraction. Zoneplates consist of alternating transparent and opaque zones of 
increasing radius and decreasing width around a central opaque zone. As a result, the exiting angle for constructive interference from each zone is slightly different, which gives zoneplates a focusing effect. The resolution of a zoneplate is determined by the width $\Delta r$ of the outermost zone and is given by $1.22 \cdot \Delta r_{\text {outer }}[14,16]$.

\subsubsection{Lamellar Multilayer Gratings}

In this thesis the combination of a ML mirror with a grating structure, a socalled Lamellar Multilayer Grating (LMG), was investigated. As previously mentioned, the spectral resolution of ML mirrors in the SXR region is determined by $N_{\text {eff }}$ and is inherently limited due to absorption [4]. The effective density, i.e. the lateral average, of each individual bi-layer can be reduced by fabricating a grating structure into a ML mirror as schematically shown in figure 1.4. This reduction in effective density also reduces absorption per bi-layer and, hence, allows the SXR radiation to penetrate deeper into the multilayer stack. As a result, $N_{\text {eff }}$ is increased and the spectral and angular resolution of the ML mirror is improved.

Although the effective density can also be reduced using other structures, such as random holes, gratings have various practical benefits. Most importantly, gratings allow for control of the diffraction as they are periodic elements that are well-defined by the grating period $D$ and the lamel width $\Gamma D$. Such parameters can easily be varied and compared between various samples, which is also important for fabrication, simulation and measurement purposes.

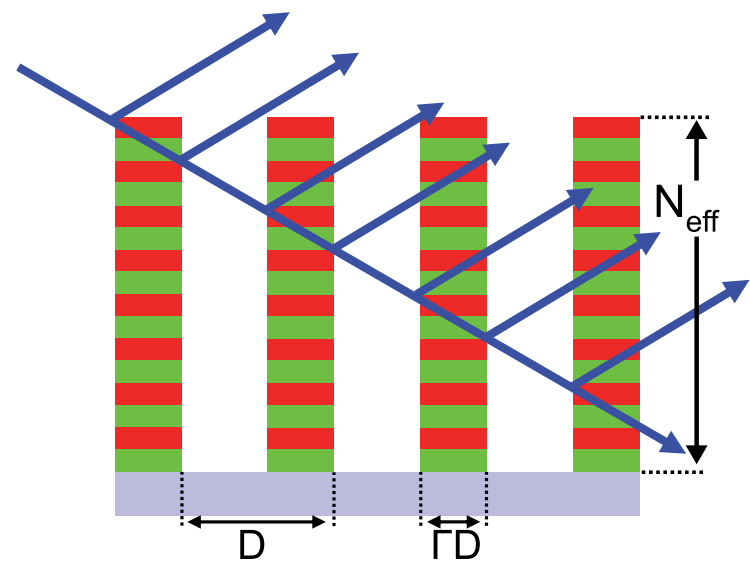

Figure 1.4: Schematic of an LMG, where the ML is defined as in figure 1.1 and the grating structure is defined by the grating period $D$ and the lamel width $\Gamma D$. 


\subsection{Applications}

The main application of LMGs is x-ray fluorescence spectroscopy. However, the general class of Bragg-Fresnel optics can be used in a much wider range of potential applications, such as x-ray imaging in microscopes, synchrotron beamlines and astronomy. The patterning of ML mirrors can also be used in EUV lithography to separate EUV and infrared radiation. These applications are briefly discussed below.

\subsubsection{X-ray fluorescence spectroscopy}

$\mathrm{X}$-ray fluorescence spectroscopy $(\mathrm{XRF})$ is a technique that is used to determine the presence and concentration of elements in a sample. This identification is achieved by measuring characteristic x-rays emitted from inner shell electron transitions. Hard x-rays with energies up to tens of $\mathrm{keV}$ are used to knock out inner shell electrons from atom's in the sample. The resulting vacancy is (primarily) filled by electrons from the next higher shell. This results in an energy loss for the electron, which is released as a secondary, or fluorescent, x-ray photon. As the energy of this fluorescent photon is determined by the difference in binding energies of the electron shells, the emitted photons have very characteristic energies that depend on the specific atom and typically have narrow natural widths of $E / \Delta E \approx 1000$ [8]. The energy range to be analyzed is thus determined by the atomic properties and specifically lighter elements emit many characteristic x-rays in the SXR spectral range due to their electronic configuration [23].

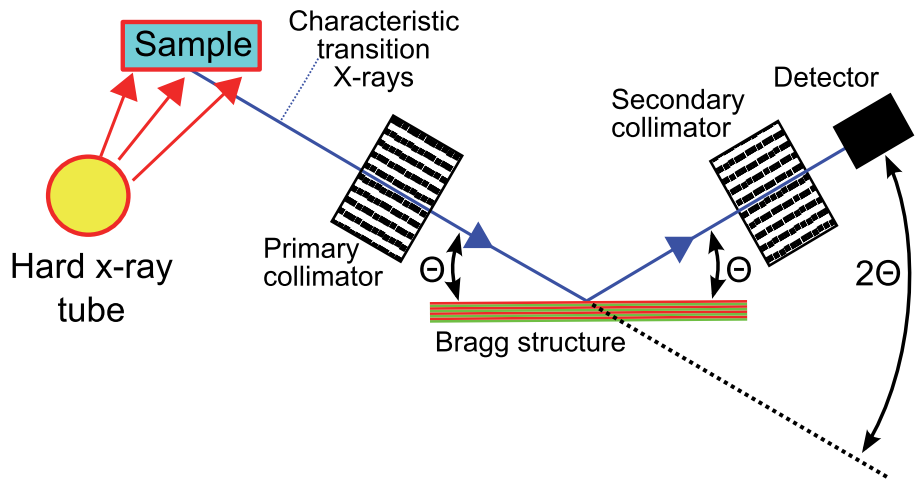

Figure 1.5: Schematic overview of an x-ray fluorescence (XRF) setup. Characteristic transition soft $\mathrm{x}$-rays from a sample are excited using hard x-rays and analyzed using a Bragg structure.

As schematically shown in figure 1.5, XRF uses an x-ray tube to produce a 
primary beam of high energy hard x-rays that irradiates a sample to knock out the inner shell electrons. The resulting emitted fluorescent radiation is then analyzed using a wavelength dispersive Bragg structure, typically a natural crystal or ML mirror, and an x-ray detector. ML mirrors used in the soft $\mathrm{x}$-ray spectral range generally can achieve spectral resolutions in the order of 50-100 [13]. However, various elements and isotopes have closely spaced shell emission lines that require spectral resolutions of at least a few hundred to be resolved [24]. As discussed in section 1.2.3, LMGs can provide such improved spectral resolution and can therefore be highly beneficial for the identification of elements in XRF.

\subsubsection{X-ray imaging}

Another possible application for the general class of Bragg-Fresnel optics are xray microscopes. Such microscopes are devices comparable to standard visible light microscopes, except for the wavelength at which they are operated. They use various optical elements to focus light from a source onto a sample and then image that sample onto a detector. The major advantage of using x-rays can be seen in Rayleigh's resolution criterion, which states the minimum feature size $(\Delta x)$ that one can still distinguish is given by [25]:

$$
\Delta x=\frac{0.610 \lambda}{N A}
$$

where $N A$ is the numerical aperture of the optical system. The factor 0.610 originates from the principal properties of a perfect lens [14] and is often replaced by an empirical constant for complex optical systems. From this equation, it can be seen that by reducing the wavelength, one can significantly improve the resolution.

Unfortunately, Rayleigh's criterion is not the only relevant factor in imaging. For instance, a certain amount of optical contrast between different parts of the sample is also required as well as sufficient transmission through the sample. A very important example is the so-called water window between the oxygen and carbon absorption edges $(\lambda=2.4-4.4 \mathrm{~nm})$, in which biological samples can be imaged very well [26]. In this region, the penetration depth of SXR through water is about $10 \mu \mathrm{m}$, which is just enough to transmit through a complete cell. Using x-ray microscopes, high-resolution images of complete and (at the moment of exposure) living cells can be obtained. Although other techniques, such as electron microscopy, can also produce very high resolution images, they require the cell to be cut into small slices and therefore loose information with respect to the actual working of a living cell.

Comparable optical elements to those used in soft x-ray microscopes [27-30] are also used in hard x-ray imaging at synchrotron beamlines [31-35] and in various astronomy applications [36-38]. These applications can thus all benefit from the possibility to pattern ML mirrors with diffractive structures to obtain Bragg-Fresnel optics. 


\subsubsection{EUV lithography}

A final important application where patterning of ML mirrors may be of interest is EUV lithography (EUVL). EUVL is an emerging technology to produce denser computer memory and faster processor chips. To achieve this, the critical dimensions of the transistors in these chips need to be as small as possible. These critical dimensions are again determined by the Rayleigh criterion (Eq. (1.6)), resulting in a continuous search for lithography techniques at shorter wavelengths to create smaller transistors. For this, the EUV wavelength of choice is $13.5 \mathrm{~nm}$, which has been selected because of its proximity to the $\mathrm{Si}-L$ absorption edge. This allows for the use of ML mirrors with silicon spacers and high reflectivities. Indeed, Mo/Si ML mirrors have a theoretical reflectivity of $74 \%$ and a value of $70.5 \%$ has already been experimentally demonstrated [39]. Although this reflectivity is already very close to the theoretical maximum, up to eleven optical elements are used in the EUV imaging system resulting in a very low overall transmission of $<3 \%$.

In the last years, several EUVL systems have been shipped to various test facilities and producers. However, these devices are mainly used to test the production process as these machines are not yet economically feasible due to the limited throughput. A major challenge for improving the throughput is the limited power available from current EUV sources. Most promising is a source that uses a $\mathrm{CO}_{2}$-laser to heat and ionize tin droplets that subsequently emit EUV. Unfortunately, this means the EUV needs to be collected and separated from the $10.6 \mu \mathrm{m}$ infrared radiation (IR) coming form the $\mathrm{CO}_{2}$-laser.

A possible solution to this challenge is to fabricate optical elements for the $10.6 \mu \mathrm{m}$ radiation into ML mirrors for the EUV range. For example, the $10.6 \mu \mathrm{m}$ radiation can be separated from the EUV by covering the collimator ellipsoid with a Fresnel zone reflector as shown in figure 1.6 [40]. The $10.6 \mu \mathrm{m}$ radiation is then focused at a different position, i.e. not at the intermediate focus, as compared to the EUV and be considerably attenuated by the exit aperture at the intermediate focus. In principle, the $10.6 \mu \mathrm{m}$ radiation can actually be re-used by designing the setup such that the focus coincides with the plasma. The focused $\mathrm{CO}_{2}$ radiation is then also used to heat the plasma which can significantly improve the efficiency of the source [40]. The principles of IR suppression with limited EUV reflection loss was recently demonstrated using a phase-shift grating [41].

\subsection{Thesis outline}

In chapter 2, we present the experimental setups used throughout this thesis. Here, we first discuss the setups used to optically characterize our Lamellar Multilayer Gratings. Next, the setups used to characterize the results of LMG fabrication are presented.

We then discuss the Coupled Waves Analysis (CWA) derived to theoret- 


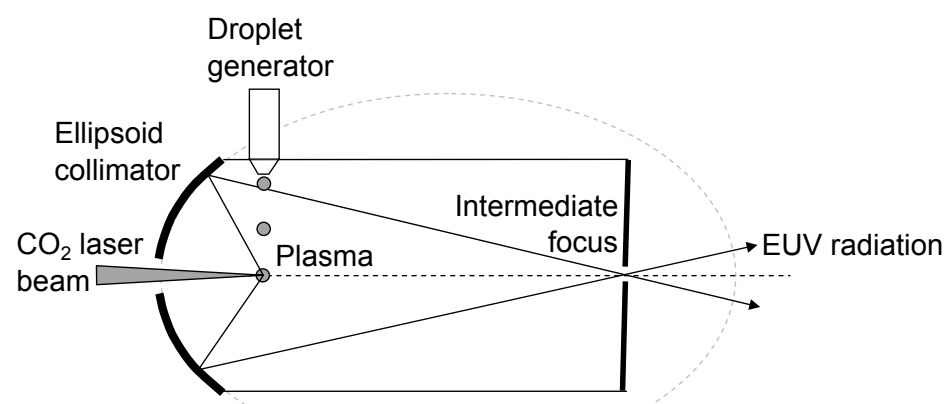

Figure 1.6: Schematic of separation of $\mathrm{CO}_{2}(\lambda=10.6 \mu \mathrm{m})$ and $\mathrm{EUV}(\lambda=$ $13.5 \mathrm{~nm})$ radiation for EUV lithography. The collimator reflects and focuses the $13.5 \mathrm{~nm}$ radiation at the intermediate focus, whereas the $10.6 \mu \mathrm{m}$ radiation is focused at a different position by a zoneplate structure fabricated in the collimator $[40]$.

ically describe LMGs in chapter 3. Compared to previously used simulation methods, this CWA significantly reduces computation time and improves physical understanding of LMGs. Based on this CWA, we derived conditions for optimal LMG reflection, the so-called single-order operation. In this regime, the incident beam excites only a single diffraction order and, hence, is not affected by diffraction losses.

Single-order operating LMGs require sub-micron grating periods with lamel widths in the order of $100 \mathrm{~nm}$. In chapter 4 we describe accurate and robust LMG fabrication in W/Si ML mirrors using UV-NanoImprint Lithography and Bosch Deep Reactive Ion Etching (DRIE). Criteria for acceptable values of fabrication artifacts, such as the typical sidewall scalloping due to the Bosch DRIE process, were determined based on a theoretical analysis. The ML structure itself was used to successfully fabricate LMGs that fulfilled these criteria. A Transmission Electron Microscope image of an LMG fabricated using our process can be seen in figure 1.7a.

SXR reflection measurements where then performed on the LMG structures fabricated using this Bosch DRIE process, as is presented in chapter 5. These measurements include the first demonstration of single-order LMG operation, clearly showing the reduced bandwidth together with increased peak reflectivity that can be achieved in this regime. The measurements were in good agreement with our theoretical calculations, showing a good understanding of the main underlying physical properties. The use of higher diffraction orders in singleorder LMG operation was also examined, now also allowing the use of higher diffraction orders to be considered for practical applications.

From the reflectivity measurements, we determined that the accuracy of 


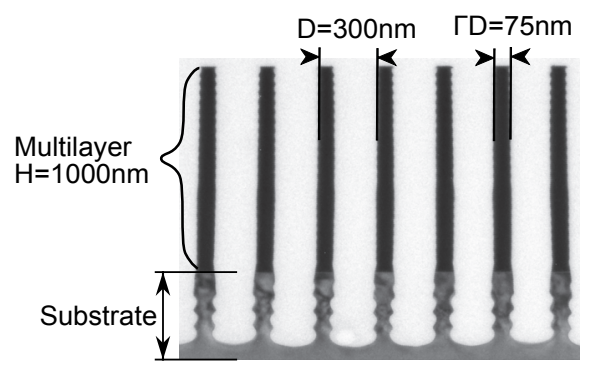

(a)

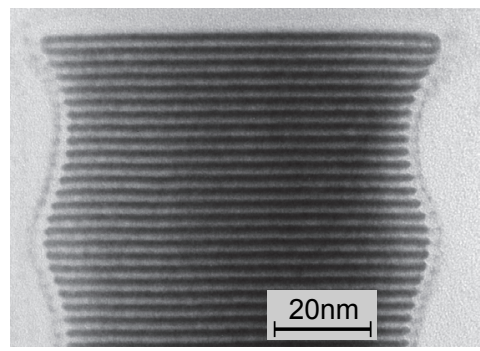

(b)

Figure 1.7: Transmission Electron Microscope image of a fabricated LMG structure. (a) A general overview of an LMG with a grating period of $300 \mathrm{~nm}$ and a lamel width of $75 \mathrm{~nm}$ fabricated in a 400 bi-layer W/Si ML mirror. (b) Zoomed image of the top of a lamel, showing the exposed sides of the ML where enhanced degradation by surface effects or internal ML structure changes can occur.

the ML deposition was limiting the achieved bandwidth and peak reflectivity for our LMGs. In chapter 6 we therefore investigated the optical performance of LMGs considering various practical limitations. In particular, the optical effect of insufficient layers to fully reflect the incident beam was determined based on a comparison of LMG optical performance simulations assuming a semi-infinite and finite ML stack. From this analysis, parameters that can be used to tailor peak reflectivity and bandwidth for LMGs with finite ML stacks were determined. An experimental demonstration of the tailoring of LMG optical performance was achieved by measuring the reflectivity of various LMGs fabricated in two different W/Si ML mirrors.

The lifetime of LMG structures is of importance to their usability in practical applications. As can be seen in figure 1.7b, the fabrication process directly exposes the ML structure to degradation processes and also significantly increases the surface area between the ML and vacuum where such processes can occur. We therefore investigated degradation effects due to fabrication and a 23 month storage in a $1 \mathrm{~atm}$ air environment as discussed in chapter 7 . Degradation processes were separated in surface phenomena, such as sidewall contamination, and internal changes to the ML structure due to thermal annealing and oxygen diffusion. The stability of SXR reflectivity was also investigated for the duration of the storage period. A preliminary study into the use of capping layers to protect patterned MLs was also performed.

Finally, conclusions on ML patterning for the fabrication of Bragg-Fresnel optics for the SXR and XUV wavelengths are discussed in chapter 8. 


\section{2 \\ Experimental}

\subsection{Introduction}

The investigation presented in this thesis required characterization of optical performance, in terms of bandwidth and peak reflectivity, and of the multilayer (ML) structure of Lamellar Multilayer Gratings (LMG). These characterizations were mainly performed using soft and hard x-ray reflectometry as presented in section 2.2. The fabrication of LMGs required the patterning of sub-micron period gratings in W/Si multilayer (ML) mirrors. Various aspects of the fabrication process were analyzed using profile measurement, imaging and spectroscopic techniques as discussed in section 2.3.

\subsection{Optical performance and multilayer struc- ture characterization}

Soft and hard x-ray reflectometry was used to analyze LMG optical performance and ML structure. Although both techniques work using similar principles, different information was obtained. Soft x-ray (SXR) reflectometry was used to characterize LMG optical performance, in terms of bandwidth and peak reflectivity, and hard x-ray (HXR) reflectometry was used to analyze the ML structure.

\subsubsection{Soft x-ray reflectometry}

A soft x-ray (SXR) reflectometer is schematically shown in figure 2.1 and is in principle the same as the analysis part of the XRF setup of figure 1.5. To 
accurately characterize LMG optical performance, a reflectometer is required that does not affect the measured reflectivity curves. For instance, the spectral bandwidth of the incident beam should be well below the spectral selectivity of our LMG structures to ensure the measured bandwidth is fully determined by the LMG structure. The absolute value of the peak reflectivity should also be measured, requiring a well-calibrated incident beam intensity and detector sensitivity. The alignment in both spatial and angular coordinates is also very important to ensure correct positioning of the beam on the sample and accurate determination of the angular peak position.

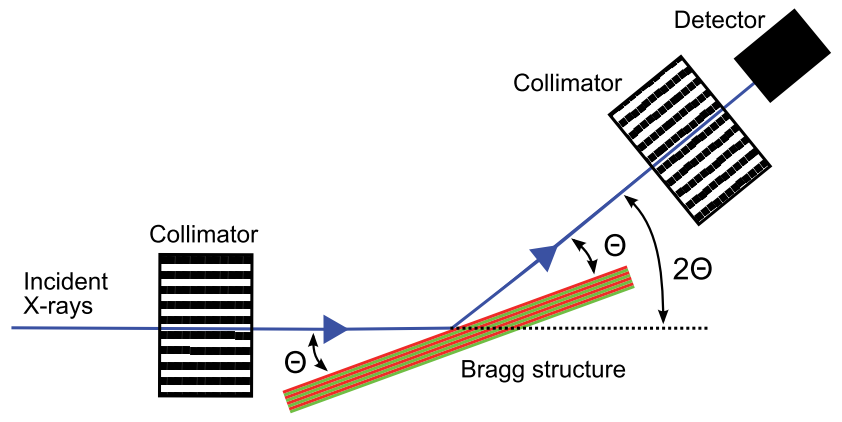

Figure 2.1: Schematic of a $\Theta-2 \Theta$ goniometric setup as used in SXR reflectometry.

The SXR reflectivity measurements presented in this work were performed at the SX-700 reflectometer of the Physikalisch-Technischen Bundesanstalt (PTB) beamline at BESSYII. The incident beam is produced from the Bremsstrahlung spectrum of a bending magnet in the electron storage ring. A plane grating monochromator is then used to select photons with a specific energy. This reflectometer achieves an energy precision below $0.1 \mathrm{eV}$, resulting in a spectral selectivity at $1 \mathrm{keV}$ of 10000 , which is well above the expected spectral selectivities of a few hundred for our LMG structures. The peak reflectivity can be measured with an accuracy of $0.2 \%$. Absorption in the beamline and measurement chamber are minimized by using base pressures in the $10^{-8}$ mbar region. Incidence angles can be varied between 0.8 and $90^{\circ}$ with a position accuracy of $\leq 3 \mu \mathrm{m}$ and angular accuracy $\leq 0.001^{\circ}$. The divergence of the incident beam is $1 \mathrm{~mm} / 8 \mathrm{~m} \sim 0.2 \operatorname{mrad}\left(0.007^{\circ}\right)$ and the detector has an acceptance angle of $4.5 \mathrm{~mm} / 0.550 \mathrm{~m} \sim 10 \mathrm{mrad}\left(0.47^{\circ}\right)$ [34, 42-45].

\subsubsection{Grazing incidence x-ray reflectometry}

Grazing incidence x-ray reflectometry (GIXR) works on the same principle as SXR reflectometry and is mainly used to characterize the ML structure. In GIXR the reflection of hard x-rays, usually at the $\mathrm{Cu}-K \alpha$ energy of $8 \mathrm{keV}$ $(\lambda=0.15 \mathrm{~nm})$, is measured for small incidence angles $\left(0-10^{\circ}\right)$. 
From these measurements, the bi-layer period can be determined from the peak positions using the corrected Bragg condition (eq. (1.2)). Below the critical angle $\Theta_{c}$ given by $\approx \sqrt{2 \bar{\delta}}$ the incident beam effectively only 'sees' the average material density of the top few nm, allowing for an accurate determination of the ML layer material composition [46, 47]. More information about the layered structure, such as roughness and compound formation can be obtained from simulations as derived in this work (see chapter 3) or software packages like IMD [48].

GIXR measurements can also be performed in-situ, for instance during annealing tests. A reference GIXR spectrum is measured and the substrate is then heated to the desired temperature via a hotplate in an air environment. This hotplate is capable of reaching temperatures between 25 and $900{ }^{\circ} \mathrm{C}$ with a $0.5^{\circ} \mathrm{C}$ accuracy. The change in $\mathrm{ML}$ bi-layer period was determined by comparing the angular positions of Bragg peaks with the reference positions. An accurate determination is achieved by considering relative shifts, measuring the same Bragg orders during annealing as for the reference and using both low and high Bragg orders [17].

\subsection{LMG structure characterization}

Various aspects of LMG fabrication were analyzed using height measurement, imaging and spectroscopic techniques. Height measurements were used to compare etching of a W/Si ML mirror and were performed using a stylus profilometer as presented in section 2.3.1. Electron imaging techniques, discussed in sections 2.3.2 and 2.3.3, were used to optimize the fabrication process and determine the spatial distribution of elements in LMGs. Finally, x-ray photoelectron spectroscopy was used to analyze the composition of the sidewfls after LMG fabrication as discussed in section 2.3.4.

\subsubsection{Stylus profilometry}

When performing etch experiments, a Dektak stylus profilometer was used as a first means of determining etch results. This technique works by moving a stylus with a tip radius of approximately $2.5 \mu \mathrm{m}$ over the sample surface and measuring height differences as function of position. The horizontal resolution is determined by various parameters of which the scan speed and height range are the most important. In the used setup, the resolution was $0.1 \%$ of the measurement range and could reach approximately $6 \mathrm{~nm}$ under optimal conditions [49].

\subsubsection{Scanning electron microscopy}

Etch results, in particular of nanostructures, were mostly characterized using a Scanning Electron Microscope (SEM). In a SEM, an electron beam is typically 
produced via thermionic emission from an electron gun fitted with a tungsten filament cathode and has an energy ranging from $0.2 \mathrm{keV}$ to several tens of $\mathrm{keV}$. This beam is then focused using condenser lenses to a spot size of about 0.4 to $5 \mathrm{~nm}$. The beam is then scanned over the surface of the sample in a raster pattern, where these primary electrons interact with the sample. Both scattered and secondary electrons can be used for imaging [50]. In this thesis, a LEO 1550 high-resolution SEM was used to image fabricated LMG structures. Samples were placed under a $70^{\circ}$ tilt, meaning the height of the structure in the image is underestimated by $7 \%$.

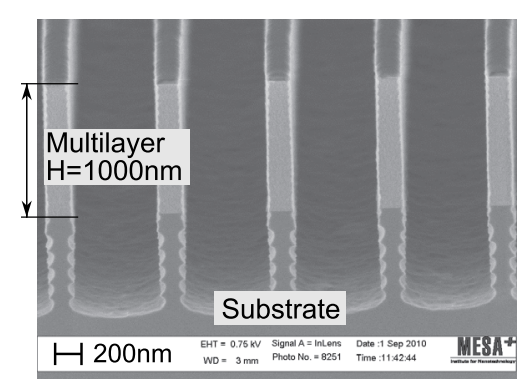

(a)

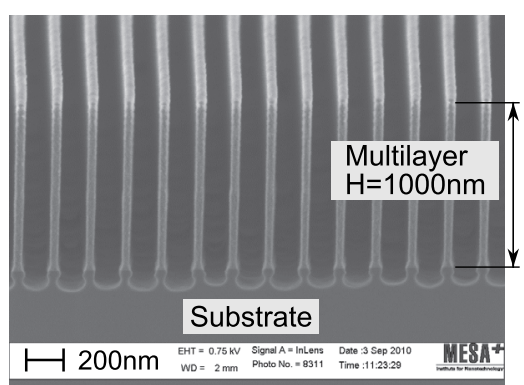

(b)

Figure 2.2: SEM images of two LMG structures etched into a $1000 \mathrm{~nm}$ $\mathrm{W} / \mathrm{Si}$ ML structure with (a) a grating period of $800 \mathrm{~nm}$ and a lamel width of $200 \mathrm{~nm}$ and (b) a grating period of $200 \mathrm{~nm}$ and a lamel width of $60 \mathrm{~nm}$.

\subsubsection{Transmission electron microscopy}

Transmission Electron Microscopy (TEM) is an electron microscopy technique whereby a beam of high energy electrons is transmitted through a very thin $(\leq 100 \mathrm{~nm})$ sample. The image is created from the interaction of the electron beam with the sample. The transmitted electron beam is magnified and focused onto an imaging device, such as a CCD camera. Due to the very small de Broglie wavelength of electrons, TEM's can image details up to the level of single columns of atoms [51].

In this work, we present TEM images made in bright field and energyfiltered (EF-TEM) operation, of which examples are shown in figure 2.3. Bright field imaging is the most common mode of operation, where the image contrast is created from differences in electron transmission at various positions on the sample. Area's on the sample that are thicker or consist of a material with a higher atomic number (high- $Z$ ) will appear darker then area's that are thinner or consist of lower- $Z$ materials. The actual image is made from a projection of the sample at the position of a detector, such as a CCD camera.

The spatial distribution of elements in the sample can be determined using 
the energy-filtered operating mode. In this mode, the image is made using electrons that have lost a specific amount of energy, due to various elastic and inelastic scattering processes, when passing through the sample. In particular, inner shell ionization is an inelastic scattering process which results in an element specific energy loss. Element sensitive imaging can then be achieved by using a magnetic prism with an adjustable slit to only allow electrons with a certain energy range to be used to create the image. To remove the background, two images are recorded using electrons that are just below the absorption edge and compared with an image using electrons at the maximum of the absorption peak. As can be seen in figure 2.3b, where the presence of silicon and oxygen are indicated by green and red, respectively, the position of various elements can then be easily determined.

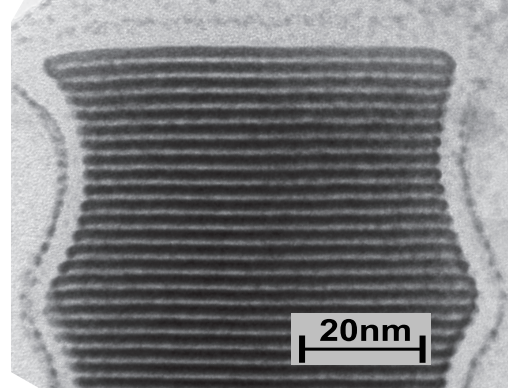

(a)

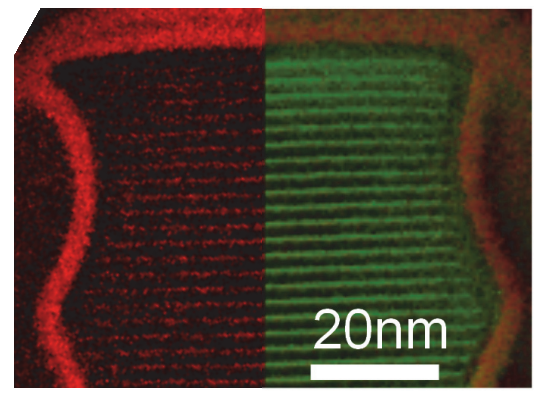

(b)

Figure 2.3: Examples of TEM images of the same structure in (a) bright field and (b) energy-filtered operation. In the energy-filtered image, green indicates silicon (only on the right-hand side of the image) and red indicates oxygen.

\subsubsection{X-ray photoelectron spectroscopy}

To determine the sidewall composition of LMGs, X-ray Photoelectron Spectroscopy (XPS) was used. XPS is a non-destructive technique that uses photoionization to determine the elemental composition and chemical state by precisely measuring the binding energies of elements in a sample. The binding energies are element specific and depend on the exact electron configuration, thereby also allowing identification of compounds based on shifts in the measured binding energies [52].

An XPS measurement is performed by irradiating a sample with a beam of $\mathrm{x}$-rays, in our case at the $\mathrm{Al}-K \alpha$ energy of $1486.6 \mathrm{eV}$, and then measuring the kinetic energy of the emitted photo-electrons. The binding energy is determined from the difference between the incident energy and the kinetic energy, which is measured using a hemispherical analyzer and corrected for the work function of the spectrometer. The photo-emitted electrons are quickly recaptured within 
either the sample or in air, meaning XPS requires ultra-high vacuum (UHV) conditions. Furthermore, XPS can only be used to analyze the top few nm of a sample as photo-emitted electrons from deeper in the same cannot escape the sample [53].

A typical XPS spectrum thus shows the number of counted electrons plotted against the binding energy. The absolute energy calibration of an XPS measurement is very important in order to determine the exact position of characteristic lines from which elements or compounds (from shifts in binding energy) can be identified. The calibration is mostly done using commonly present elements with well-known characteristic lines, such as aliphatic carbon $\mathrm{C} 1 s$ with a characteristic line at an energy of $284.8 \mathrm{eV}$. Atomic concentrations can be determined from the number of counted electrons and, hence, from the peak area. However, the number of detected electrons for each element should first be divided by the empirically determined relative sensitivity factor (RSF) of that element and subsequently be normalized over all detected elements.

As an example, a measured XPS spectrum of the W4f binding, which consists of a double peak or doublet, of partly oxidized tungsten is shown in figure 2.4. The measurement is fitted using well known peaks with fixed widths and, as this is a doublet, fixed relative intensity and relative shift between the peaks within the doublet. The intensity of the doublet and positions of both doublets is then fitted to result in a minimum residual of the sum of both doublets. In this measurement, a first doublet is found at the binding energies of 29 and $33 \mathrm{eV}$, indicating pure tungsten, and a second doublet is found at 35 and $38 \mathrm{eV}$, which is typical for oxidized tungsten. Based on the relative peak area of the doublets, $70 \%$ of the measured tungsten can be determined to be oxidized.

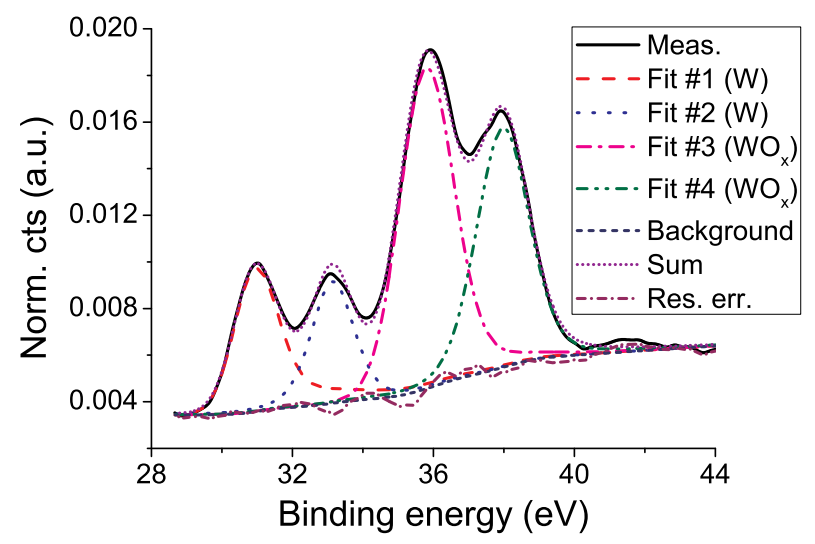

Figure 2.4: Example of a measured XPS spectrum (W4f doublet) of partly oxidized tungsten. In this example, $70 \%$ of the measured tungsten can be concluded to be oxidized. 


\section{3 \\ Theory of Lamellar Multilayer Gratings}

\subsection{Introduction}

For the design of Lamellar Multilayer Gratings (LMGs) with optimal performance, in terms of bandwidth and peak reflectivity, an adequate theory for the modeling of the diffraction and reflection of the incident soft x-ray (SXR) beam is required. At present, several rigorous approaches such as modal theory or integral method are used to simulate LMGs [54-57], in particular in the SXR region [11, 12, 58-63]. However, the modal theory is poorly suited for LMGs with non-rectangular groove shapes or for smooth interfaces between neighboring materials. Although the integral method described in ref. [57] overcomes these problems, it is also stated that it does not suffice for the modeling of gratings coated by hundreds of layers.

In the present chapter we describe the results of a novel LMG performance analysis using a Coupled Waves Approach (CWA) that does not have the aforementioned limitations. The equations of this method are based on a general expansion of the reflected field in terms of different order diffracted waves and describe the changes in amplitude of various incident and reflected waves through a system of coupled equations. This approach can be used for arbitrary lamellar shapes, arbitrary distributions of the dielectric constant in the multilayer (ML) structure and without limitations on the grating period, lamel width and number of bi-layers in the ML structure. The method is well suited for the analysis of LMG performance in the SXR region as the diffracted peaks are narrow in this region and therefore the number of diffraction orders that overlap, and hence can influence each other, is limited. Note that CWA has been used before successfully in SXR optics for calculations of sliced multilayer 
gratings, zone plates and holographic structures [57, 59, 64].

Using the CWA presented here, we derive that for small lamel widths LMGs operate in a single-order regime in which there is no significant overlap of the $0^{t h}$ diffraction order with higher orders. Only the $0^{\text {th }}$ order then needs to be considered when calculating LMG specular reflection, allowing a two-wave approximation to be used. We show that the reflection of a SXR wave from an LMG operating in this regime simply equals the reflection from a ML mirror with a material density that is decreased with a factor equal to lamel-width-tograting-period ratio $\Gamma$. Sophisticated diffraction theories are thus not necessary for the proper calculation of LMG performance in the single-order regime. In contrast to what was stated in [12], we demonstrate that it is possible to derive novel analytic design criteria for LMGs operating in the single-order regime. We also show that the resolution of an LMG operating in single-order regime is not limited by absorption, in contrast to the resolution of a conventional ML mirror. A high resolution and high reflectivity have been shown to be mutually exclusive for a ML mirror [4], whereas the resolution of an LMG is only limited by technological factors and the peak reflectivity can still be as high as for a conventional ML mirror.

In this chapter, we first derive and study the CWA in section 3.2. We begin with the derivation of basic equations in section 3.2.1, assuming an ideal lamel profile. More generalized equations that can be used to describe non-ideal lamel shapes and include various sidewall effects are stated next in section 3.2.2. In sections 3.2.3 and 3.2.4, both a numerical and analytical solution to the basic CWA equations are presented. From the analytical solution, a generalized Bragg condition is derived in section 3.2.5 and the validity of this CWA for different polarizations is investigated in section 3.2.7. Using the CWA results, a single-order operating regime has been identified, which significantly simplifies simulations and provides optimal LMG optical performance for practical applications. This operating regime is discussed in detail in section 3.3.

\subsection{Coupled waves approach}

\subsubsection{Basic equations}

In this section, we derive the basic equations of the Coupled Waves Approach (CWA). We first define the parameters of an LMG and its geometrical representation as shown in figure 3.1a. Here, an incident beam from the left (In), under grazing angle $\Theta_{0}$, is reflected from the multilayer and diffracted into multiple orders (Out) by the grating structure. The multilayer is built up from $N$ bi-layers with thickness $d$. Each bi-layer consists of an absorber material $(A)$ with thickness $\gamma d$ and a spacer material $(S)$ with thickness $(1-\gamma) d$. The grating structure of the LMG is defined by the grating period $D$ and lamel width $\Gamma D$.

For simplicity, the basic equations assumed a rectangular lamel shape, al- 


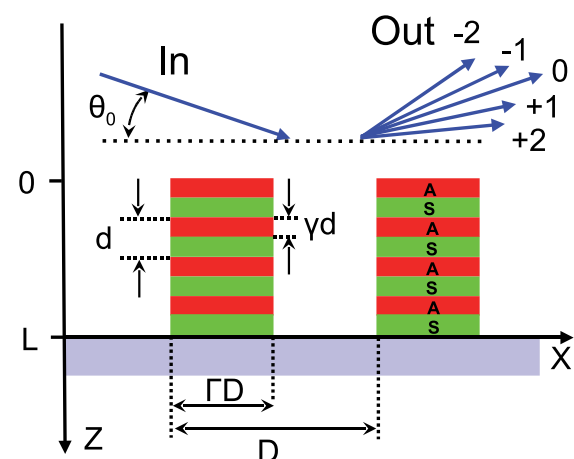

(a)

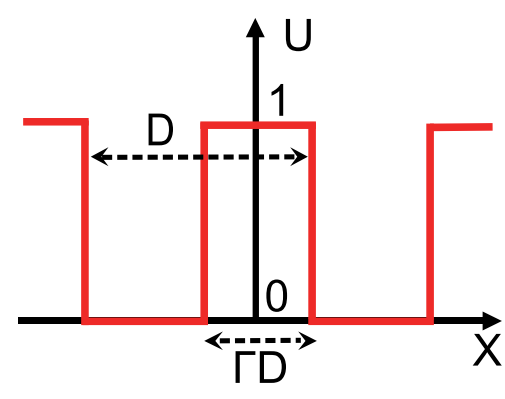

(b)

Figure 3.1: (a) Schematic of SXR diffraction from an LMG. An incident beam from the left $(I n)$, under grazing angle $\Theta_{0}$, is reflected from the multilayer and diffracted into multiple orders (Out) by the grating structure. The multilayer is built up of $N$ bi-layers with thickness $d$. Each bi-layer consists of an absorber material $(A)$ with thickness $\gamma d$ and a spacer material $(S)$ with thickness $(1-\gamma) d$. The grating structure of the LMG is defined by the grating period $D$ and lamel width $\Gamma D$. (b) Function $U(x)$ describing the lamel profile.

though the approach described below can be applied to any lamellar shape. The Z-axis is defined as directed into the depth of the substrate, $L$ is the total thickness of the multilayer structure and the step-like periodic function $U$, shown in figure 3.1b, describes the lamellar profile. The spatial distribution of the dielectric constant is then written as follows:

$$
\begin{array}{rll}
\epsilon(x, z)=1 & \text { if } & z<0 \\
\epsilon(x, z)=1-\chi(z) U(x, \Gamma, D) & \text { if } & 0 \leq z \leq L \\
\epsilon(x, z)=\epsilon_{\text {sub }} & \text { if } & z>L
\end{array}
$$

where the function $\chi(z)$ is the complex susceptibility, which varies with depth in the multilayer structure. The susceptibility is proportional to the atomic polarizability [65], which describes the induced dipole moment of a material when an electric field is applied. Compared to visible optics, our definition of the dielectric constant $\epsilon$ is $1-\chi$ to indicate $\epsilon$ is generally below 1 .

The function $U$ can be expanded into the Fourier series:

$$
U(x, \Gamma, D)=\sum_{n=-\infty}^{+\infty} U_{n} e^{2 i \pi n x / D}
$$

with $U_{0}=\Gamma$ and $U_{n \neq 0}=\sin (\pi n \Gamma) /(\pi n)$. Please note that eq. (3.2) differs to that used in refs. $[5,6]$ as the point $x=0$ was now chosen to be in the center 
of the lamel instead of at the left side of the lamel. Shifting the coordinates of the previous works by $x^{\prime}=x-\Gamma D / 2$ yields the same coefficients as used in this work.

For brevity, we only considered reflection of s-polarized radiation incident onto the LMG perpendicular to the lamels (i.e. in the plane of figure 3.1a) and the effect of interfacial roughness was neglected. The effect of p-polarized radiation is discussed in section 3.2.7. To analyze the diffraction pattern, the 2D-wave equation was solved:

$$
\nabla^{2} E(x, z)+k^{2} \epsilon(x, z) E(x, z)=0,
$$

where the dielectric constant $\epsilon$ is a periodic function of $x$ and is described by eqs. (3.1) and (3.2) and $k$ is the wavenumber given by $2 \pi / \lambda$. The general solution then has the following form (chapter 1 in ref [66]):

$$
F_{n}^{\prime \prime}(z)+k_{n}^{2} F_{n}(z)=k^{2} \chi(z) \sum_{m} U_{n-m} F_{m}(z)
$$

where $F_{n}$ represents the field of the $n^{\text {th }}$ order diffraction wave. The boundary conditions of this system signify that only plane waves from the vacuum are incident onto the LMG at the grazing angle $\Theta_{0}$ :

$$
F_{n}^{\prime}(0)+i \kappa_{n} F_{n}(0)=2 i \kappa_{n} \delta_{n, 0} ; \quad F_{n}^{\prime}(L)-i \kappa_{n}^{(s)} F_{n}(L)=0 .
$$

Here, $\kappa_{n}=\sqrt{k^{2}-q_{n}^{2}}$ and $\kappa_{n}^{(s)}=\sqrt{k^{2} \epsilon_{s u b}-q_{n}^{2}}$ are the z-components of the wave vectors for the $n^{\text {th }}$ diffraction order in vacuum and substrate, respectively, where $q_{n}=q_{0}+2 \pi n / D$ with $q_{0}=k \cos \Theta_{0}$ and $\delta_{n, 0}$ is the Kronecker symbol.

From eq. (3.4) it can be seen that the interaction of the incident and diffracted waves with the multilayer structure is described through the complex susceptibility $\chi(z)$, which may be an arbitrary function, e.g. periodic or aperiodic, step-like or smooth, etc. It can also be seen that the diffracted waves of different orders interrelate with each other and with the incident wave through the coefficients $U_{n}$ which characterize the lamellar profile. In the case of a rectangular lamellar shape, the coefficients $U_{n}$ are numbers. For more general lamellar profiles, e.g. trapezoidal, the form of eq. (3.4) remains the same, but the coefficients become a function of $z$.

As a first test of this approach, eqs. (3.4) and (3.5) were used to calculate the reflection of a conventional multilayer mirror by inserting $\Gamma=1$. All coefficients $U_{n}$ then become zero, except the coefficient $U_{0}$ which then equals 1 . Eq. (3.4) is then reduced to the simplest equation:

$$
F_{0}^{\prime \prime}(z)+\kappa_{0}^{2} F_{0}(z)=k^{2} \chi(z) F_{0}(z)
$$

which is indeed an ordinary $1 \mathrm{D}$ wave equation describing the reflection of a wave from a conventional ML mirror, as would be expected.

Eq. (3.4) can be solved using either a numerical or, under certain approximations, an analytical method. Although the numerical method is more generally 
valid, the analytical solution has several advantages. Most importantly, the analytical solution allows for a preliminary physical analysis directly from the derived equations as presented in section 3.2.4.

\subsubsection{Generalized equations}

To analyze the influence of non-ideal lamel shapes on LMG reflection, we deduced generalized CWA equations. For this, we considered a non-ideal lamel profile as is typically the result of fabrication and which is shown schematically in figure 3.2. Here, curves 1 and 2 are the sidewalls of the lamel, such that the space between these curves is filled by a ML structure. In addition, a layer of thickness $h$ on the sidewalls of the lamel, indicated by curves 3 and 4, was introduced. Here, we refer to these layers as being cover layers as the theory describing these layers is valid for both contamination layers as well as deliberately grown capping layers used to protect LMG structures. Curves 1 and 3 as well as curves 2 and 4 are assumed for simplicity to be equidistant, while curves 1 and 2 (as well as 3 and 4) are mirror inverted with respect to the Z-axis. Suppose the right side wall depicted by curve 2 is described by a single-valued function $x=p(z)$. Then $x=-p(z)$ can be written for the opposite wall, i.e. curve 1 , and $x=\mp p(z) \mp h$ can be written for curves 3 and 4 , respectively. It was assumed that the lamel width and cover layer thickness are sufficiently small such that $2(p(z)+h)$ does not exceed the grating period $D$ at any $z$.

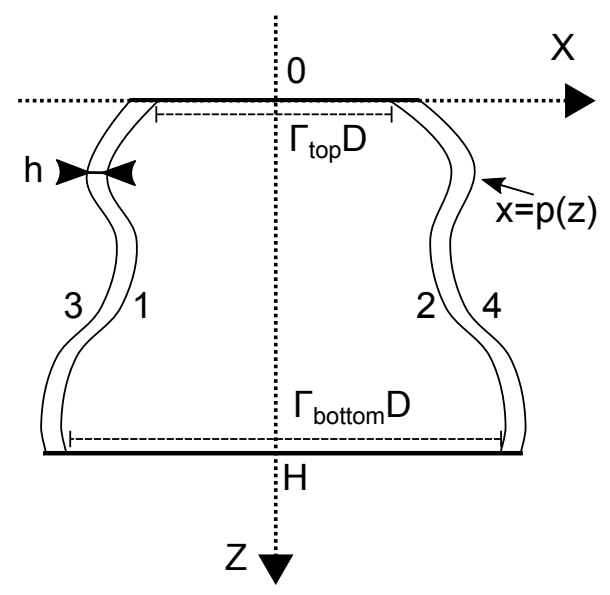

Figure 3.2: Schematic cross section of a general shaped lamel. Curves 1 and 2 indicate the side walls of the ML structure and are described by the function $p(z)$. A cover layer with thickness $h$ is indicated by curves 3 and $4 . \Gamma_{\text {top }}$ and $\Gamma_{\text {bottom }}$ indicate the $\Gamma$-ratio at the top and bottom of the lamel, respectively. 
The spatial distribution of the dielectric constant is then written as:

$$
\begin{gathered}
\epsilon(x, z<0)=1 ; \quad \epsilon(x, z>H)=1-\chi_{\text {sub }} \\
\epsilon(x, 0 \leq z \leq H)=1-\chi(z) U(x, z)-\chi_{\operatorname{cov}} V(x, z)
\end{gathered}
$$

where $\chi(z)$ is the susceptibility of a multilayer structure varying with the depth, while $\chi_{c o v}$ and $\chi_{s u b}$ are the constant susceptibility of the cover layer and substrate, respectively. The function $U(x, z)=1$ if the point $(x, z)$ is placed inside the multilayer structure and $U=0$ otherwise. Similarly, the function $V(x, z)=1$ if the point $(x, z)$ is placed inside the cover layer and otherwise equals 0 .

Assuming the dielectric constant to be a periodic function of $x$, we represented the functions $U$ and $V$ as the Fourier series:

$$
\begin{aligned}
& U(x, z)=\sum_{n=-\infty}^{+\infty} U_{n}(z) e^{2 i \pi n x / D} \\
& V(x, z)=\sum_{n=-\infty}^{+\infty} V_{n}(z) e^{2 i \pi n x / D}
\end{aligned}
$$

where

$$
\begin{gathered}
U_{n}(z)=\frac{1}{\pi n} \sin \left(\frac{2 \pi n p(z)}{D}\right) \\
V_{n}(z)=\frac{2}{\pi n} \sin \left(\frac{\pi n h}{D}\right) \cos \left[\pi n\left(\frac{2 p(z)}{D}+\frac{h}{D}\right)\right]
\end{gathered}
$$

with $U_{0}=2 p(z) / D$ and $V_{0}=2 h / D$.

Following the steps also used in section 3.2.1, the Rayleigh expansion of the field was written as $[5,66]$ :

$$
\begin{gathered}
E(x, z)=\sum_{n=-\infty}^{+\infty} F_{n}(z) e^{i q_{n} x} ; \quad q_{n}=q_{0}+\frac{2 \pi n}{D} \\
q_{0}=k \cos \Theta_{0} ; \quad k=\frac{2 \pi}{\lambda}
\end{gathered}
$$

and eqs. (3.8)-(3.10) were substituted into the wave equation $\nabla^{2} E(x, z)+$ $k^{2} \epsilon(x, z) \cdot E(x, z)=0$. An infinite system of coupled waves equations was then obtained:

$$
F_{n}^{\prime \prime}(z)+\kappa_{n}^{2} F_{n}(Z)=k^{2} \sum_{m}\left[\chi(z) U_{n-m}(z)+\chi_{\operatorname{cov}} V_{n-m}(z)\right] F_{m}(z)
$$

for $n=0, \pm 1, \pm 2, \ldots$ with the same boundary conditions as eq. (3.5). This system can be used to analyze the optical effect of various imperfections arising from LMG fabrication as discussed in sections 4.4 and 7.2.1. 


\subsubsection{Numerical solution}

Both systems of eqs. (3.4) and (3.11) can be solved numerically. Here, we demonstrate the numerical solution of eq. (3.4) as this system represents an idealized LMG structure and therefore has slightly simpler equations.

We represented the field of the $n^{t h}$ order diffraction wave $F_{n}(z)$ as a superposition of two waves propagating in different directions along the z-axis.

$$
F_{n}(z)=A_{n}(z) e^{i \kappa_{n} z}+B_{n}(z) e^{-i \kappa_{n} z}
$$

Here, the functions $A_{n}(z)$ and $B_{n}(z)$ can vary with depth and can be considered to be the amplitudes of a transmitted and reflected wave, respectively. For a unique determination we imposed additional requirements to the functions $A_{n}(z)$ and $B_{n}(z)$ in a manner typical for the coupled waves approach:

$$
A_{n}^{\prime}(z) e^{i \kappa_{n} z}+B_{n}^{\prime}(z) e^{-i \kappa_{n} z}=0 .
$$

Substituting (3.12) and (3.13) in eq. (3.4) a system of first order differential equations for the amplitudes $A_{n}$ and $B_{n}$ was obtained:

$$
\begin{aligned}
\frac{d A_{n}(z)}{d z}=-\frac{i k^{2}}{2 \kappa_{n}} \chi(z) \sum_{m=-\infty}^{+\infty} U_{n-m}[ & A_{m}(z) e^{i\left(\kappa_{m}-\kappa_{n}\right) z} \\
& \left.+B_{m}(z) e^{-i\left(\kappa_{m}-\kappa_{n}\right) z}\right] \\
\frac{d B_{n}(z)}{d z}=+\frac{i k^{2}}{2 \kappa_{n}} \chi(z) \sum_{m=-\infty}^{+\infty} U_{n-m}[ & A_{m}(z) e^{i\left(\kappa_{m}+\kappa_{n}\right) z} \\
& \left.+B_{m}(z) e^{-i\left(\kappa_{m}+\kappa_{n}\right) z}\right]
\end{aligned}
$$

where $n=0, \pm 1, \ldots, \pm \infty$. The boundary conditions were then written as:

$$
A_{n}(0)=\delta_{n, 0} \quad \text { and } \quad B_{n}(L)=0
$$

where we put the dielectric permeability of the substrate to unity, i.e. neglected the effect of the substrate on the reflection of an incident wave. This is, at least for soft x-rays, a quite reasonable assumption as the refractive index of materials is close to 1. Eqs. (3.14) is fully equivalent to the second order system (3.4), but is more preferable for numerical calculations.

Evidently, the amplitudes $r_{m}$ and $t_{m}$ of the waves diffracted into vacuum and into the depth of the substrate were found by solving eqs. (3.14) with boundary conditions (3.15) to be:

$$
r_{n}=B_{n}(0), \quad t_{n}=A_{n}(L)
$$

To test the validity of the CWA derived in this section, we compared the calculated $0^{\text {th }}$ order reflectivity curves with other works $[5,12,63]$. We therefore 
considered a Mo/B ${ }_{4} \mathrm{C} \mathrm{ML}(d=6 \mathrm{~nm}, \gamma=0.33, N=150)$ in which a grating is fabricated with a period $D$ of $2 \mu \mathrm{m}$ and a $\Gamma$ of 0.3 . The incident SXR energy was $E=183.4 \mathrm{eV}$ and the values of the complex susceptibility $\chi=1-\epsilon$ used for calculations were $\chi_{M o}=2.61 \cdot 10^{-2}-i \cdot 5.77 \cdot 10^{-3}$ and $\chi_{B_{4} C}=$ $4.43 \cdot 10^{-3}-i \cdot 1.08 \cdot 10^{-3}$. The calculations included up to the \pm 7 diffraction order. As can be seen in figure 3.3, the agreement between our numerical solution and the previous results of ref. [63] are in excellent agreement.

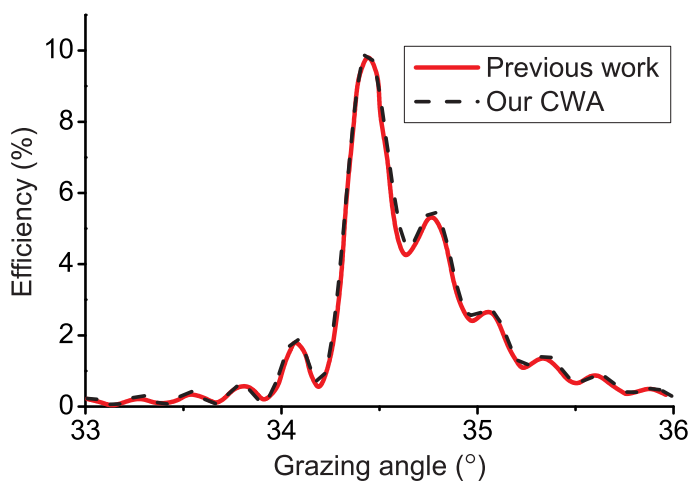

Figure 3.3: Calculated $0^{\text {th }}$ order diffraction efficiency of a Mo/B ${ }_{4} \mathrm{C}$ LMG versus the incidence grazing angle using our numerical solution (dotted line) and compared to previous results (solid line) of ref. [63].

\subsubsection{Analytical solution}

Eq. (3.4) can also be solved analytically for the specific case where the incident beam only excites a single diffraction order as discussed next. The validity of this assumption is discussed in section 3.3.

\section{Zeroth order efficiency}

To derive an analytical solution for the $0^{\text {th }}$ order diffraction efficiency, also referred to as the reflectivity, we used a two-wave approximation and only considered the incident and specularly reflected waves. The higher order terms in eqs. (3.14) were therefore neglected leading to:

$$
\begin{aligned}
& \frac{d A_{0}(z)}{d z}=-\frac{i k^{2}}{2 \kappa_{0}} \Gamma \chi(z)\left[A_{0}(z)+B_{0}(z) e^{-2 i \kappa_{0} z}\right] \\
& \frac{d B_{0}(z)}{d z}=+\frac{i k^{2}}{2 \kappa_{0}} \Gamma \chi(z)\left[A_{0}(z) e^{+2 i \kappa_{0} z}+B_{0}(z)\right]
\end{aligned}
$$

where $A_{0}(0)=1$ and $B_{0}(L)=0$. 
To solve these equations, we considered a periodic multilayer structure having abrupt interfaces and consisting of two materials, namely a spacer and an absorber, with susceptibilities $\chi_{S}$ and $\chi_{A}$, respectively. The susceptibility of the ML structure is then described by

$$
\chi(z)=\chi_{S}+\left(\chi_{A}-\chi_{S}\right) u(z),
$$

where the step-like function $u(z)$ is similar to the function $U$ that describes the lamellar profiles:

$$
u(z, \gamma, d)=\sum_{n=-\infty}^{+\infty} u_{n} e^{2 i \pi n z / d}
$$

with $u_{0}=\gamma$ and $u_{n \neq 0}=\left(1-e^{-2 i \pi n \gamma}\right) /(2 i \pi n)$.

We limited ourselves to the most important case of an incident wave onto the multilayer within or near the Bragg resonance of the $j^{\text {th }}$ order, i.e. we supposed that $j \lambda \approx 2 d \sin \Theta_{0}$ or, equivalently, $\kappa_{0} \approx \pi j / d$. By substituting eqs. (3.18) and (3.19) into eqs. (3.17), the following system was obtained:

$$
\begin{aligned}
\frac{d A_{0}(z)}{d z} & +\frac{i k^{2}}{2 \kappa_{0}}\left[\bar{\chi} \Gamma A_{0}(z)\right. \\
& \left.+\left(\chi_{A}-\chi_{S}\right) u_{j} \Gamma B_{0}(z) e^{2 i\left(\pi j / d-\kappa_{0}\right) z}\right]=\Delta A(z) \\
\frac{d B_{0}(z)}{d z} & -\frac{i k^{2}}{2 \kappa_{0}}\left[\bar{\chi} \Gamma B_{0}(z)\right. \\
& \left.+\left(\chi_{A}-\chi_{S}\right) u_{j} \Gamma A_{0}(z) e^{-2 i\left(\pi j / d-\kappa_{0}\right) z}\right]=\Delta B(z)
\end{aligned}
$$

where $\bar{\chi}=\chi_{A} \gamma+\chi_{S}(1-\gamma)$ is the mean susceptibility of the multilayer structure. The left-hand side of eqs. (3.20) contains all terms that vary slowly with $z$. The functions $\Delta A$ and $\Delta B$ on the right-hand side denotes all terms oscillating quickly with $z$, which, as they only weakly influence the amplitudes $A_{0}$ and $B_{0}$, can be neglected.

A system of coupled differential equations with constant coefficients was then obtained by introducing $a_{0}(z) \equiv A_{0}(z) \cdot e^{-i\left(\pi j / d-\kappa_{0}\right) z}$ and $b_{0}(z) \equiv B_{0}(z)$. $e^{i\left(\pi j / d-\kappa_{0}\right) z}$ :

$$
\begin{aligned}
\frac{d a_{0}(z)}{d z}+ & i\left(\frac{\pi j}{d}-\kappa_{0}+\frac{k^{2}}{2 \kappa_{0}} \bar{\chi} \Gamma\right) a_{0}(z) \\
& +i \frac{k^{2}}{2 \kappa_{0}}\left(\chi_{A}-\chi_{S}\right) \Gamma u_{j} b_{0}(z)=0 \\
\frac{d b_{0}(z)}{d z}- & i\left(\frac{\pi j}{d}-\kappa_{0}+\frac{k^{2}}{2 \kappa_{0}} \bar{\chi} \Gamma\right) b_{0}(z) \\
- & i \frac{k^{2}}{2 \kappa_{0}}\left(\chi_{A}-\chi_{S}\right) \Gamma u_{-j} a_{0}(z)=0
\end{aligned}
$$


with the same boundary conditions $a_{0}(0)=1$ and $b_{0}(L)=0$ as for eqs. (3.17). By solving eqs. (3.21), an analytical expression for the $0^{\text {th }}$ order diffraction efficiency was obtained:

$$
R_{0}=\left|\frac{B_{-} \tanh (S N d)}{b \tanh (S N d)-i \sqrt{B_{+} B_{-}-b^{2}}}\right|^{2}
$$

where $R_{0}=\left|r_{0}\right|^{2}$. The terms used in eq. (3.22) are:

$$
\begin{gathered}
b=\bar{\chi} \Gamma+2 \sin \Theta_{0}\left(\frac{j \lambda}{2 d}-\sin \Theta_{0}\right) \\
B_{ \pm}=\left(\chi_{A}-\chi_{S}\right) u_{ \pm j} \Gamma ; \quad S=\frac{k}{2 \sin \Theta_{0}} \sqrt{B_{+} B_{-}-b^{2}}
\end{gathered}
$$

where the Bragg parameter $b$ characterizes a deviation from the Bragg resonance, $B_{ \pm}$describes the modulation of the ML structure and $S$ characterizes the variation in amplitudes $A_{0}$ and $C_{0}$ with $z$.

\section{Higher diffraction order efficiencies}

Using a similar approach as for the analytical solution of the $0^{\text {th }}$ order diffraction efficiency, the higher order efficiencies could also be solved analytically. We considered the $m^{\text {th }}$ order diffraction efficiency, where we limited ourselves to angles and wavelengths close to the Bragg resonance (quasi-Bragg resonance), i.e. assumed $j \lambda \approx d\left(\sin \Theta_{0}+\sin \Phi_{m}\right)$, where $\Phi$ is the diffraction angle, or equivalently $\kappa_{0}+\kappa_{m}=2 \pi j / d$. Here, the index $j$ represents the Bragg reflection order by the multilayer and the index $m$ represents the diffraction order by the grating structure.

Using a similar two-wave approximation as for the $0^{\text {th }}$ order, it can be seen from eqs. (3.14) that the amplitudes $A_{0}(z)$ and $B_{m}(z)$ only interrelate resonantly with each other and all other equations can be neglected. Then, similar to the $0^{t h}$ order analytic solution, we introduced $a_{0}(z) \equiv A_{0}(z)$. $e^{-i\left(\pi j / d-\left(\kappa_{0}+\kappa_{m}\right) / 2\right) z}$ and $b_{m}(z) \equiv B_{m}(z) \cdot e^{i\left(\pi j / d-\left(\kappa_{0}+\kappa_{m}\right) / 2\right) z}$ and obtained a system of differential equations with constant coefficients:

$$
\begin{aligned}
& \frac{d a_{0}(z)}{d z}+i\left(\frac{\pi j}{d}-\frac{\kappa_{0}+\kappa_{m}}{2}+\frac{k^{2}}{2 \kappa_{0}} \bar{\chi} \Gamma\right) a_{0}(z) \\
&+ i \frac{k^{2}}{2 \kappa_{0}}\left(\chi_{A}-\chi_{S}\right) U_{-m} u_{j} b_{0}(z)=0 \\
& \frac{d b_{0}(z)}{d z}-i\left(\frac{\pi j}{d}-\frac{\kappa_{0}+\kappa_{m}}{2}+\frac{k^{2}}{2 \kappa_{0}} \bar{\chi} \Gamma\right) b_{0}(z) \\
&-i \frac{k^{2}}{2 \kappa_{0}}\left(\chi_{A}-\chi_{S}\right) U_{m} u_{-j} a_{0}(z)=0
\end{aligned}
$$


Solving eqs. (3.24), we found an expression for the diffraction efficiency $R_{m} \equiv\left|r_{m}\right|^{2} \operatorname{Re}\left(\kappa_{m} / \kappa_{0}\right)$, which has the same form as eq. (3.22), but has somewhat different terms as those of eq. (3.23):

$$
\begin{gathered}
b=\frac{\sin \Theta_{0}+\sin \Phi_{m}}{2 \sqrt{\sin \Theta_{0} \sin \Phi_{m}}} \bar{\chi}+ \\
2 \sqrt{\sin \Theta_{0} \sin \Phi_{m}}\left(\frac{j \lambda}{2 d}-\frac{\sin \Theta_{0}+\sin \Phi_{m}}{2}\right) \\
B_{ \pm}=\left(\chi_{A}-\chi_{S}\right) u_{ \pm j} U_{\mp m} ; \quad S=\frac{k}{2 \sqrt{\sin \Theta_{0} \sin \Phi_{m}}} \sqrt{B_{+} B_{-}-b^{2}}
\end{gathered}
$$

As we also did for the numerical solution, we tested the validity of the analytical solution by comparing this to other works. However, as the analytical solution is only valid within our two-wave approximation, it can only be compared to our numerical solution as the required grating periods (see eq. (3.39) in section 3.3) are well below the periods used in refs. [12, 63]. Figure 3.4 shows the comparison of our numerical and analytic solutions for the same ML and $\Gamma$-ratio as used in figure 3.3, but with a reduced grating period $D$ of $300 \mathrm{~nm}$. As can be seen, the agreement between our numerical and analytical solution is excellent. Hence, in single-order operation, sophisticated diffraction theories are not necessary to properly calculate LMG reflection and diffraction.

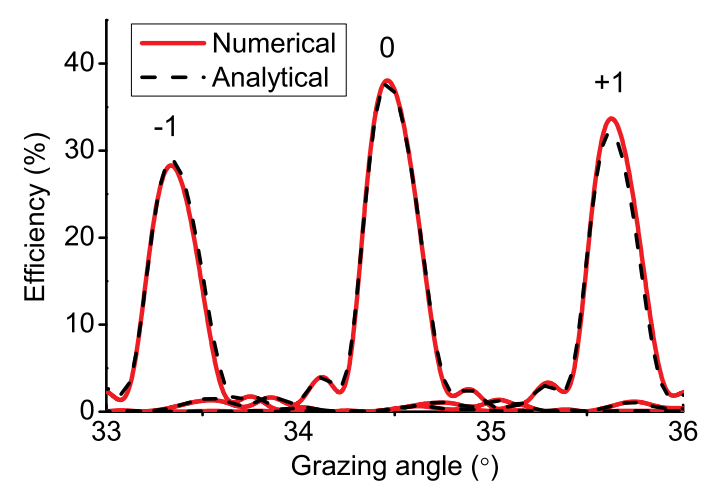

Figure 3.4: Calculated $0^{\text {th }}$ and first order diffraction efficiency of a $\mathrm{Mo} / \mathrm{B}_{4} \mathrm{C}$ LMG versus the incidence grazing angle using our analytical solution (dotted lines) and compared to the numerical calculations (solid lines). The ML and $\Gamma$ were the same as for figure 3.3 , but the grating period was reduced to $D=$ $300 \mathrm{~nm}$ to achieve single-order operation. 


\subsubsection{Analytic description of LMG optical performance}

Using the analytic solution of the previous section, we derived analytical equations describing LMG optical performance. First, we derived a generalized Bragg condition for single-order LMGs, where we assumed a semi-infinite multilayer structure $(N \rightarrow \infty)$ for simplicity. Eqs. (3.22) and (3.25) then reduce to their simplest form with $\tanh (S N d)=1$. As the Bragg peak is very narrow due to the small polarizability of matter in the SXR wavelength region, we neglected the wavelength dependence of the dielectric constant inside the peak. The reflectivity then only depends on the incidence angle and the radiation wavelength through the Bragg parameter $b$. At maximum reflectivity, we have the condition $d R_{0} / d \tau=2 \operatorname{Re}\left[r^{*}(d r / d b)(d b / d \tau)\right]$ (where either $\tau=\sin \Theta_{0}$ or $\tau=\lambda$ ) and obtained a generalized Bragg condition that also includes the absorption and refraction of radiation:

$$
\begin{aligned}
\frac{j \lambda}{2 d}= & \frac{\sin \Theta_{0}+\sin \Phi_{m}}{2}-\frac{\sin \Theta_{0}+\sin \Phi_{m}}{4 \sin \Theta_{0} \sin \Phi_{m}} \Gamma \operatorname{Re}(\bar{\chi})+ \\
& \frac{\operatorname{Re}\left(\chi_{A}-\chi_{S}\right)}{\sin \Theta_{0}+\sin \Phi_{m}} \frac{\operatorname{Im}\left(\chi_{A}-\chi_{S}\right)}{\operatorname{Im}(\bar{\chi})} \frac{\sin ^{2}(\pi j \gamma)}{(\pi j)^{2}} \frac{\sin ^{2}(\pi m \Gamma)}{(\pi m)^{2} \Gamma}
\end{aligned}
$$

If the condition (eq. (3.26)) is fulfilled, the diffraction efficiency achieves a peak value, which can be written in a very simple manner comparable to ref. [4]:

$$
\begin{gathered}
R_{m, \text { peak }}=\frac{1-w}{1+w} ; \\
w=\sqrt{\frac{1-y^{2}}{1+f^{2} y^{2}}} ; \quad f=\frac{\operatorname{Re}\left(\chi_{A}-\chi_{S}\right)}{\operatorname{Im}\left(\chi_{A}-\chi_{S}\right)} ; \\
y=\frac{\operatorname{Im}\left(\chi_{A}-\chi_{S}\right)}{\operatorname{Im}(\bar{\chi})} \frac{\sin (\pi j \gamma)}{(\pi j)} \frac{2 \sqrt{\sin \Theta_{0} \sin \Phi_{m}}}{\sin \Theta_{0}+\sin \Phi_{m}} \frac{\sin (\pi m \Gamma)}{(\pi m) \Gamma}
\end{gathered}
$$

Note that the peak reflectivity (eq. (3.27)) achieves its maximum possible value when the parameter $y$ is maximal and, hence, the thickness ratio $\gamma$ of a multilayer structure obeys the equation:

$$
\tan (\pi j \gamma)=\pi j\left[\gamma+\frac{\operatorname{Im}\left(\chi_{S}\right)}{\operatorname{Im}\left(\chi_{A}-\chi_{S}\right)}\right]
$$

which is well-known in the theory of conventional SXR multilayer mirrors [20, $67]$.

From eq. (3.27), it can also be seen that the peak reflectivity $(m=0)$ is independent of the grating parameters and corresponds to that of a conventional multilayer mirror [4]. This is because the parameters $f$ and $y$ entirely determine the peak reflectivity and are not changed if the density of both materials are scaled by the same factor $\Gamma$. In contrast, the penetration depth of 
the radiation into the multilayer structure $L_{M S}$, and therefore also the spectral and angular resolution of an LMG, is inversely proportional to $\Gamma$ :

$$
L_{M S}=N_{e f f} \cdot d \sim \frac{1}{S}=\frac{\lambda \sin \Theta_{0}}{\pi \Gamma \operatorname{Im}(\bar{\chi}) \sqrt{\left(1-y^{2}\right)\left(1+f^{2} y^{2}\right)}}
$$

From this equation, an approximate value for the reflection bandwidth was also derived. Following refs. [4, 18], the resolving power equals $E / \Delta E=$ $\tan \Theta / \Theta \approx N_{\text {eff }} \cdot \pi / 2$, where $N_{\text {eff }}$ was defined as in eq. (3.29). Using $d=$ $\lambda / 2 \sin \Theta$, the following bandwidth prediction was obtained:

$$
\Delta \Theta_{L M G} \approx \frac{2 \operatorname{Im}(\bar{\chi}) \Gamma}{\sin \left(2 \Theta_{0}\right)} \sqrt{\left(1-y^{2}\right)\left(1+f^{2} y^{2}\right)}
$$

From this equation, it can easily be seen that the bandwidth scales linearly with $\Gamma$. However, the dependence on other parameters is more complicated as $\bar{\chi}, f$ and $y$ all depend on the materials and ML $\gamma$-ratio.

\subsubsection{Interfacial roughness}

Interfacial roughness can have a strong effect on ML reflectivity and, hence, also on that of LMGs. Using the analytical solution of section 3.2.4, the effect of interfacial roughness in the ML structure on LMG optical performance was analyzed. As the interfacial roughness is present in the ML structure, the effect of roughness on LMG reflectivity can be described in the same manner as for a ML mirror. In general, the peak reflectivity of a ML mirror depends on the interfacial roughness parameters in a very complicated manner requiring extensive knowledge of the roughness on each individual layer to obtain a complete description. However, there are two limiting cases when the reflectivity of a rough ML mirror is expressed in a simple manner which are analyzed below. More details on the analysis of roughness on ML reflection can be found in refs. [16, 68-70].

The first limiting case is that of totally conformal roughness at different interfaces with large roughness correlation lengths in the interfacial plane. "Large" means that the width of the scattering distribution is small as compared to the bandwidth of the Bragg peak. Then, assuming the roughness height to be distributed in accordance with the normal law, the reflectivity was written as [70]:

$$
R(\Theta)=R_{0}(\Theta) e^{-\left(4 \pi \sigma_{c} \sin \Theta / \lambda\right)^{2}},
$$

where $R_{0}$ is the reflectivity of a perfectly smooth ML mirror and $\sigma_{c}$ is the root mean square (rms) value of the conformal roughness height. The exponential term is referred to as the Debye-Waller factor and is only valid within specific approximations [69, 70].

This type of roughness is considered to result in additional scattering from the interfacial plane. However, the large correlation length results in scattering 
within very small angles with respect to the Bragg peak angle. As the acceptance angle of the detectors in the measurement setups (see section 2.2) used in this work are comparatively large, this scattered radiation was still measured by the detector. As a result, long-scale conformal roughness does not affect measured reflectivity curves and was therefore not considered in the fitting of reflectivity measurements.

The second limiting case occurs when the roughness of the different interfaces is totally non-conformal, while the correlation function of each interface is the same. In addition, the correlation length in the interfacial plane is supposed to be very small (tending to zero) and the roughness heights are again distributed according to the normal law. To describe the effect of such roughness, the modulation parameter $B_{ \pm}$in eqs. 3.23 and 3.25 should be replaced by:

$$
B_{ \pm} e^{-1 / 2\left(2 \pi \sigma_{n c} / d\right)^{2}} \approx B_{ \pm} e^{-1 / 2\left(4 \pi \sigma_{n c} \sin \Theta / \lambda\right)^{2}}
$$

where $\sigma_{n c}$ is the rms value of the non-conformal roughness height and the grazing incidence angle $\Theta$ is supposed to be large as compared with the critical angle of the total external reflection of the ML structure. The effect of smallscale non-conformal roughness on the reflectivity is equivalent to that of a smooth transition layer placed between neighboring materials, i.e. the smallscale roughness results in increased transmittance of the incident wave into the ML structure and, hence, in a reduction of the reflectivity. Non-conformal roughness was therefore considered in the fitting of reflectivity measurements.

\subsubsection{CWA validity for p-polarized radiation}

In the previous sections we have only considered reflection and diffraction of s-polarized radiation by LMGs. Reflection and diffraction of p-polarized radiation was investigated by comparing the description of the dielectric constant distribution in the LMG for both polarization states.

First, the dielectric constant distribution in the LMG for s-polarized radiation was derived. In accordance with eqs. (3.1) and (3.19), this distribution can be written as a 2D Fourier series:

$$
\begin{aligned}
\epsilon(x, z)= & 1-\bar{\chi} \cdot \sum_{m} U_{m} e^{2 i \pi m x / D}- \\
& \left(\chi_{A}-\chi_{S}\right) \cdot \sum_{m} \sum_{j=0} U_{m} u_{j} e^{2 i \pi(m x / D+j z / d)}
\end{aligned}
$$

Using the two-wave approximation of section 3.2.4, only the terms with $m=0$ in the first sum and only one harmonic with fixed $m$ and $j$ in the second sum are kept. The first term $\Gamma \bar{\chi}$ describes the propagation of an incident wave through an LMG neglecting reflection and diffraction and the second term gives the main contribution to the diffraction efficiency under Bragg or quasi-Bragg resonance $j \lambda \approx d\left(\sin \Theta_{0}+\sin \Phi_{m}\right)$. In order to solve the wave equation, it is 
then sufficient to use (instead of eq. (3.33))

$$
\epsilon(x, z)=1-\bar{\chi}-\left(\chi_{A}-\chi_{S}\right) U_{m} u_{j} e^{2 i \pi(m x / D+j z / d)}
$$

For p-polarized radiation, the corresponding $2 \mathrm{D}$ wave equation is more involved due to the presence of first derivatives $\nabla^{2} H+k^{2} \epsilon H-\vec{\nabla}(\ln \epsilon) \cdot \vec{\nabla} H=0$ where $H$ is the nonzero Y-component of the magnetic field perpendicular to the plane of figure 3.1 and where $\vec{\nabla} \equiv \vec{i}_{x} \partial / \partial x+\vec{i}_{z} \partial / \partial z$. To simplify the wave equation, we introduced a new field function $\tilde{H} \equiv H(x, z) / \sqrt{\epsilon(x, z)}$, which obeys the 2D-wave equation without the first derivatives of the field $\nabla^{2} \tilde{H}(x, z)+k^{2} \tilde{\epsilon}(x, z) \tilde{H}(x, z)=0$ achieved by introducing a modified function $\tilde{\epsilon}(x, z)$. It can be shown that $\tilde{\epsilon}(x, z)$ relates to the original dielectric function as follows:

$$
\tilde{\epsilon}(x, z) \equiv \epsilon(x, z)+\frac{1}{2 k^{2}} \frac{\nabla^{2} \epsilon(x, z)}{\epsilon(x, z)}-\frac{3}{4 k^{2}} \frac{[\vec{\nabla} \epsilon(x, z)]^{2}}{\epsilon^{2}(x, z)}
$$

Expanding eq. (3.35) into a 2D Fourier series and keeping only linear terms of the small susceptibilities $\chi_{S}$ and $\chi_{A}$, we obtained, instead of eq. (3.33):

$$
\begin{aligned}
\epsilon= & 1-\bar{\chi} \cdot \sum_{m} U_{m} e^{2 i \pi m x / D}\left[1-\frac{1}{2}\left(\frac{m \lambda}{D}\right)^{2}\right]-\left(\chi_{A}-\chi_{S}\right) \cdot \\
& \sum_{m} \sum_{j=0} U_{m} u_{j} e^{2 i \pi(m x / D+j z / d)}\left[1-\frac{1}{2}\left(\frac{j \lambda}{d}\right)^{2}-\frac{1}{2}\left(\frac{m \lambda}{D}\right)^{2}\right]
\end{aligned}
$$

As before, the two-wave approximation was used to keep only specific terms from eq. (3.36):

$$
\begin{gathered}
\epsilon \approx 1-\bar{\chi} \Gamma-\left(\chi_{A}-\chi_{S}\right) U_{m} u_{j} e^{2 i \pi(m x / D+j z / d)} . \\
{\left[1-\frac{1}{2}\left(\frac{j \lambda}{d}\right)^{2}-\frac{1}{2}\left(\frac{m \lambda}{D}\right)^{2}\right] \approx} \\
\approx 1-\bar{\chi} \Gamma-\left(\chi_{A}-\chi_{S}\right) U_{m} u_{j} e^{2 i \pi(m x / D+j z / d)} \cos \left(\Theta_{0}+\Phi_{m}\right)
\end{gathered}
$$

Here, the resonance condition of diffraction $j \lambda / d \approx \sin \Theta_{0}+\sin \Phi_{m}$ and the grating equation $m \lambda / D=\cos \Phi_{m}-\cos \Theta_{0}$ were already taken into account. Eq. (3.37), which is valid for p-polarized light, was then compared with eq. (3.34) for s-polarized light. The comparison shows that all the expressions deduced in our CWA for s-polarized radiation are also valid for p-polarized radiation, if the dielectric modulation of the multilayer structure $\chi_{A}-\chi_{S}$ is replaced by $\left(\chi_{A}-\chi_{S}\right) \cos \left(\Theta_{0}+\Phi_{m}\right)$. This proves that, independent of the polarization, an analytical calculation of reflection and diffraction by LMGs is possible.

Eq. (3.37) also clearly demonstrates the main feature of p-polarized radiation reflection, namely that at the Brewster's angle of incidence $\Theta_{0}=\pi / 4$, the 
modulation effect of a multilayer structure disappears and the reflectivity (the $0^{\text {th }}$ order diffraction efficiency) goes to zero. Similarly, the $m^{\text {th }}$ order diffraction efficiency goes to zero if the diffracted beam propagates perpendicular to the incident one, i.e. at $\Theta_{0}+\Phi_{m}=\pi / 2$.

\subsection{Single-order LMG operation}

In section 3.2.4, we used a two-wave approximation to derive an analytical solution of our CWA equations. This regime is referred to as single-order operation. The validity of this approximation and the practical benefits of this operating regime are discussed next.

We first determined when single-order operation is achieved. The angular distance between neighboring diffraction orders must then be sufficiently large with respect to the bandwidth of the Bragg peak, such that the higher diffraction orders fall outside of the acceptance angle of the $0^{\text {th }}$ order. The bandwidth $\left(\Delta \Theta_{L M G}\right)$ should then be small as compared to the angular distance between neighboring peaks $d / D$. For single-order operation, eq. 3.4 was reduced to:

$$
F_{0}^{\prime \prime}(z)+\kappa_{0}^{2} F_{0}(z)=k^{2} \Gamma \chi(z) F_{0}(z) .
$$

This equation actually only differs from eq. (3.6) by the parameter $\Gamma \leq 1$, which is inserted as a multiplier of the susceptibility $\chi(z)$. As the polarizability, and hence susceptibility, in the SXR region is proportional to the material density, we concluded that eq. (3.38) describes the reflection of a wave from a conventional multilayer structure consisting of materials whose densities are effectively reduced by a factor of $\Gamma$. The bandwidth of a conventional ML mirror $\left(\Delta \Theta_{M M}\right)$ is determined by the difference in susceptibility of the materials in the ML [4] and therefore also decreases by a factor $\Gamma$, leading to $\Delta \Theta_{L M G} \approx$ $\Gamma \Delta \Theta_{M M}$. Single-order operation is therefore achieved when [5]:

$$
\Gamma D \Delta \Theta_{M M}<<d
$$

Using comparative calculations we determined that ensuring the left-hand side of eq. (3.39) is at least three-times smaller than the right-hand side yields agreements of reflectivities (two-wave approximation vs. multi-wave expansion) by better than 5 relative $\%$.

In the following, we compared LMG performance, in terms of bandwidth and peak reflectivity, with that of a conventional ML mirror. For a conventional ML mirror in the SXR spectral region, it was shown in ref. [4] that the peak value of the reflectivity is completely determined by two absorption parameters: $f=\operatorname{Re}\left(\chi_{A}-\chi_{S}\right) / \operatorname{Im}\left(\chi_{A}-\chi_{S}\right)$ and $g=\operatorname{Im}\left(\chi_{S}\right) / \operatorname{Im}\left(\chi_{A}\right)$, where $\chi_{A}$ and $\chi_{B}$ are the susceptibilities of the materials (absorber and spacer) in the multilayer structure. Unfortunately, ref. [4] also showed that for a conventional ML mirror a small bandwidth and high $0^{\text {th }}$ order efficiency are mutually exclusive. Although the bandwidth, and thus the spectral resolution, of a conventional 
ML can be improved in a number of ways, the bandwidth is eventually always limited by the absorption of the spacing material:

$$
\left(\Delta \Theta_{M M}\right)_{\min }=\frac{2 \operatorname{Im}\left(\chi_{S}\right)}{\sin \left(2 \Theta_{0}\right)}
$$

and the minimal bandwidth $\left(\Delta \Theta_{M M}\right)_{\text {min }}$ can only be obtained for a ML mirror with a near zero peak reflectivity.

However, the performance is quite different for single-order operating LMGs. From eq. (3.38), we deduced that single-order operation effectively reduces the susceptibility $(\chi(z))$, or equivalently the density, of the ML by a factor $\Gamma$ and thus reduces the bandwidth by the same factor. Evidently, such a proportional variation in the density of both materials does not change the parameters $f$ and g. Hence, the peak reflectivity of an single-order operating LMG can be the same as that of a conventional ML mirror consisting of bulk density materials. In accordance with eq. (3.29), the SXR penetration depth then also increases and the number of bi-layers should be increased by the same ratio.

Figure 3.5 illustrates these conclusions. Here, several reflectivity curves at $E=183 \mathrm{eV}$ are shown for various LMGs based on the Mo/B $4 \mathrm{C}$ ML parameters as before, but with sufficient bi-layers $(N=100 / \Gamma)$ to ensure maximum peak reflectivity is achieved. The $\Gamma=1$ curve thus represents the reflectivity curve of the conventional ML mirror, which has a bandwidth of the Bragg peak of $\Delta \Theta_{M M}=0.82^{\circ}$. The minimal bandwidth this ML system can achieve at this energy is thus equal to $\left(\Delta \Theta_{M M}\right)_{\min }=0.13^{\circ}$. The other three curves depict reflectivity curves for LMGs with various $\Gamma$-ratios. The lamellar width is the same for all three LMGs $(\Gamma D=70 \mathrm{~nm})$ and fulfills the single-order condition (3.39). As can be seen, the bandwidth clearly reduces when reducing the $\Gamma$-ratio and actually reaches $\Delta \Theta_{L M G}=0.083^{\circ}$ for $\Gamma=1 / 10$. This is roughly 10 times smaller than for the conventional ML mirror and actually about 1.5 times less than the minimal possible bandwidth for the ML system used here. The peak reflectivity is the same as that of a conventional ML mirror by increasing the number of bi-layers by $1 / \Gamma$. Finally, a shift in the position of the various Bragg peaks is visible. This shift is caused by the dependence of the Bragg condition (eq. (3.26)) on the $\Gamma$-ratio as the effective susceptibilities of both materials are scaled by this factor. Here, the effective susceptibility refers to the average susceptibility of an individual layer in the lateral direction.

In comparison, the peak reflectivity of multi-order LMGs is strongly reduced due to diffraction losses. This can be seen when comparing the $0^{\text {th }}$ order efficiency of the multi-order LMG $(10 \%)$ in figure 3.3 with that of the singleorder LMG $(38 \%)$ in figure 3.4. From these figures, it can also easily be seen that multi-order operation results in significant secondary structures adjacent to the main Bragg peak, which are also caused by the excitation of higher diffraction orders in multi-order operation.

The considerations discussed above clearly demonstrate the advantages of single-order LMG operation. In this regime, there are no physical limitations 


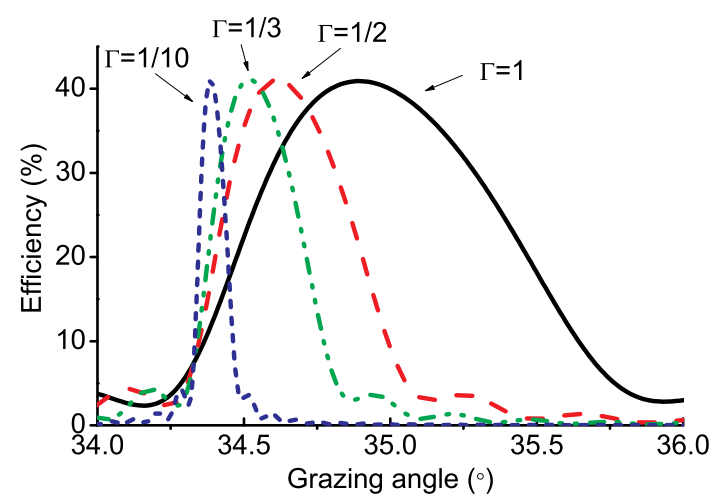

Figure 3.5: Zeroth order efficiency versus grazing angle at $E=183 \mathrm{eV}$ of a conventional $\mathrm{Mo} / \mathrm{B}_{4} \mathrm{C}$ ML mirror $(\Gamma=1, N=50)$ and three LMGs with fixed lamel widths $(\Gamma D=70 \mathrm{~nm})$, different $\Gamma$-ratios and varying number of bi-layers $(N=100 / \Gamma)$.

on the minimal bandwidth, which simply reduces by the factor $\Gamma$. At the same time, peak reflectivity is not affected as the total absorption is the same as for a conventional ML mirror and there are no diffraction losses. Of course, technological limitations of ML deposition and LMG fabrication still limit the best obtainable LMG performance.

\subsection{Conclusions}

Using our Coupled Waves Approach (CWA), we have identified a high-resolution, high-reflectivity single-order operating regime for Lamellar Multilayer Gratings (LMG) for the soft-x-ray (SXR) spectral range. In this single-order regime, the overlap of the $0^{\text {th }}$ diffraction order with higher orders is negligible. The performance, in terms of bandwidth and peak reflectivity, of LMGs operating in the single-order regime can be calculated assuming a conventional multilayer (ML) mirror of which the material densities have been reduced by the same fraction as the factor $\Gamma$ (lamel-width-to-grating-period ratio). For LMGs operating in single-order, the bandwidth scales with $\Gamma$ and the number of bi-layers $N$ required for maximum reflectance scales with $1 / \Gamma$ in comparison to a conventional ML mirror. We have also shown that the resolution and reflectivity of a single-order LMGs are only limited by the number of bi-layers $N$ and the lamel width-to-period ratio $\Gamma$ that can be obtained in practice. A single-order LMG can thus reach much higher resolutions than a conventional ML mirror, without loss of peak reflectivity. To determine achievable LMG optical performance, the technology of LMG fabrication is investigated in the next chapter. 


\section{4 \\ Fabrication of gratings in $\mathrm{W} /$ Si multilayer mirrors}

\subsection{Introduction}

The fabrication of single-order LMG structures requires the etching of nanoscale features in ML mirrors. As EUV and soft x-ray wavelengths (figure 3.1a) are only a few nm, the ML stack has a bi-layer period $d$ of comparable size. Furthermore, the bi-layers consist of two different materials that generally react very differently to the etching process. Due to the low refractive index contrast, the total ML stack generally consists of up to hundreds of bi-layers, resulting in total ML stack heights of $\geq 1 \mu \mathrm{m}$. For single-order LMG operation, the required lamel aspect-ratios are in the order of $\geq 10: 1$, which is well above previously achieved values of $\sim 5: 1$ [71]. As these structures are used as optical elements, the fabrication should not negatively affect the optical behavior and consequently leave the nanoscale layered arrangement intact.

Although Reactive Ion Etching (RIE) has been used to etch ML mirrors, this approach generally suffers from a lack of directional etching, resulting in poor critical-dimension (CD) control and a limitation on ML stack heights that can be etched $[63,71]$. Furthermore, these processes showed strong dependence of the etch results on the grating structure. Although Bosch Deep Reactive Ion Etching (Bosch DRIE) offers a directional pattern transfer, the cyclic nature of Bosch DRIE results in a typical sidewall scalloping of which the optical effect may be unacceptable. Furthermore, the effect of the thin-layered structures with dual-material composition, in particular the absorber layer, on etch results is unknown.

In this chapter, we demonstrate accurate and robust etching of $\mathrm{W} / \mathrm{Si} \mathrm{ML}$ 
mirrors for the fabrication of Lamellar Multilayer Gratings (LMG). For costeffective fabrication of LMGs in small series, UV-NanoImprint Lithography was used to define the grating structures. A Bosch DRIE process was developed to transfer this pattern into a ML stack. Using this process, we have reproducibly fabricated LMGs with $200 \mathrm{~nm}$ grating periods and lamel aspect-ratios of $\sim 17: 1$. The high aspect-ratio was achieved by using the layered structure of the ML. This layered structure suppressed scallop formation, which was subsequently used to achieve low tapers with minimal dependence on feature dimensions.

The experimental procedure is first discussed in section 4.2. Next, a description of the Bosch etch process and scallop formation is given in section 4.3. A generalized Coupled Waves Approach was used to determine fabrication criteria for LMGs, as is presented in section 4.4. Finally, the effect of the ML layered structure on etch results is investigated in section 4.5, where the main focus is on profile taper and scalloping.

\subsection{Experimental}

In this work, $400 \mathrm{~mm}$ silicon wafers were coated with $400 \mathrm{~W} / \mathrm{Si}$ bi-layers $(d-$ spacing of $2.53 \mathrm{~nm}$ and $\gamma$ of 0.28 ) [72]. A single imprint template with 27 different grating structures, having grating periods between 200 and $2000 \mathrm{~nm}$ and lamel widths between 60 and $1350 \mathrm{~nm}$, was used to define various LMG structures. This template was used for UV-NanoImprint Lithography (UV-NIL) in an Imprio 55 system from Molecular Imprints with the S-FIL ${ }^{T M}$ process [73]. Before imprinting, a transfer layer of $60 \mathrm{~nm}$ DUV 30J was spincoated on the ML wafer at $3000 \mathrm{rpm}$ and then baked at $120^{\circ} \mathrm{C}$ for $120 \mathrm{~s}$. In the imprint machine, a silicon containing imprint material was locally dispensed on top of the transfer layer. The template with the desired grating structures was then pressed into the imprint material with a force of $3 \mathrm{~N}$ at room temperature. The imprint resist was cured by a $3 \mathrm{~s}$ UV exposure while the template was still in contact. Finally, the template was detached leaving its negative shape in the hardened imprint material. The patterned layer showed a feature height of $100 \mathrm{~nm}$ and a residual layer of $40 \mathrm{~nm}$ [74]. Samples between 10x10 mm² and $30 \times 30 \mathrm{~mm}^{2}$ were then cleaved from the imprinted $400 \mathrm{~mm}$ ML wafer and an etch mask was prepared using a fluorine-based Reactive Ion Etching (RIE) process [74]. The sample was then fixed on a blank silicon carrier wafers using a small droplet of Fomblin ${ }^{T M}$ vacuum oil for ML etching. All temperatures used in the fabrication process were $\leq 120^{\circ} \mathrm{C}$ to prevent annealing of the ML. This temperature was concluded to be acceptable as only a $<0.1 \%$ d-spacing change was found in the ML after $60 \mathrm{hrs}$ at this temperature as is discussed in more detail in section 7.2.2.

For the ML etching, we used a fluorine based Bosch DRIE process in an Adixen Alcatel AMS100SE. This device has been described in detail in ref. [75]. Such a process is feasible as both components of the multilayer react volatile with fluorine [76-78]. To determine the ML etch process, the fabrication of 
silicon nanostructures was investigated first for general process development. Subsequently, the etching of the ML was compared with silicon etching. Based on the silicon study and the comparison with ML etching, a ML etch process was determined. Although only W/Si MLs were investigated in this work, this approach should be generally valid for any ML structure consisting of materials that have volatile reaction products with fluorine.

After ML etching, samples that were to be characterized using soft x-ray reflectometry were cleaned of organic contaminants and the passivation layer. This cleaning was performed using a brief $\mathrm{O}_{2}$ plasma for 1 min at $20 \mathrm{~W} R \mathrm{R}$ power in a parallel-plate RIE system. Samples for visual characterization were cleaved and imaged using a LEO 1550 high-resolution scanning electron microscope under a $70^{\circ}$ tilt.

\subsection{Etching and scalloping in Bosch DRIE}

The pattern transfer into the ML was performed using Bosch Deep Reactive Ion Etching (DRIE). This technique uses sequential etch and passivation steps to achieve a directional etch. In this study, the passivation layer used was a fluorine carbon (FC)-layer formed by a $\mathrm{C}_{4} \mathrm{~F}_{8}$ plasma (figure 4.1a) [75, 79, 80]. This passivation layer prevents further lateral etching during subsequent etching cycles. After the passivation deposition, an etch step is performed (figure $4.1 b_{1}+b_{2}$ ). At the beginning of the etch step, the passivation layer is directionally removed at the bottom of the opening through physical etching by a bombardment of ionic, mainly $\mathrm{SF}_{x}^{+}$, species (figure $4.1 b_{1}$ ). For silicon samples, a (semi-)isotropic etching is performed using the fluorine radicals from the plasma (figure $4.1 b_{2}$ ) once the passivation layer has been directionally removed. This sequence is then repeated many times (figure $4.1 c$ ) to obtain the desired (nano)structures. Profile taper is determined by the position at which the isotropic etching can begin and therefore depends on the passivation end point. The cyclic nature of Bosch DRIE also results in typical side-wall scalloping as can be seen schematically in figure $4.1 c$.

Figure 4.2 schematically shows an etched (nano)structure, which is characterized by a number of parameters. First of all, the etch rates $(E R)$ are determined by dividing the etch depth by the etch time. The selectivity is then given by the ratio between the substrate and mask etch rates. The etching generally varies as features become deeper and the trench aspect-ratio $(A R)$ is therefore defined as the depth in the trench divided by the mask opening. When investigating the etching, additional focus is given to profile taper and scalloping caused by the Bosch DRIE process. Profile taper is the angle of the sidewall, where a positive taper means that the structure is narrower at the top than at the bottom and vice versa for a negative taper. The taper is determined from the top $\left(T W_{t o p}\right)$ and bottom $\left(T W_{\text {bottom }}\right)$ trench widths and the etch depth. Finally, the typical scallops of Bosch DRIE are characterized by the scallop width $a$, scallop length $l$ and scallop ratio given by $a / l$. 

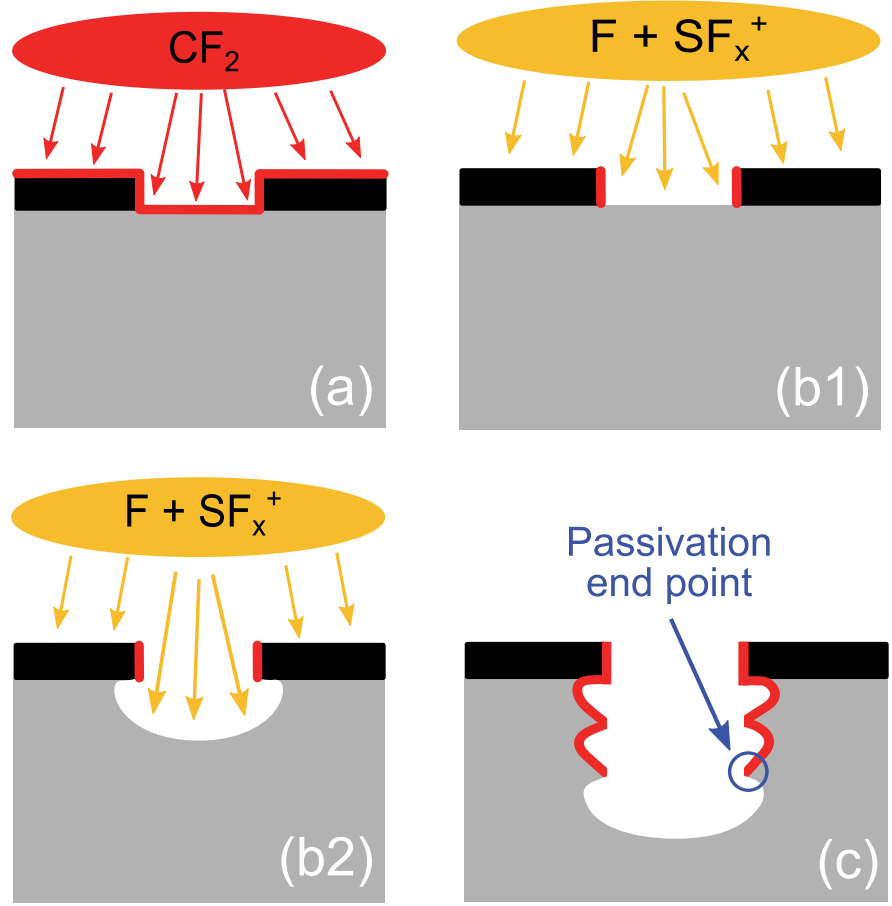

Figure 4.1: Schematic of the various steps in Bosch DRIE. (a) A FCpassivation layer is deposited. (b1) At the beginning of the etch cycle, the passivation layer is partially removed by the incident ion flux with an energy and angular distribution $N(E, \alpha)$. (b2) As soon as the passivation layer is sufficiently removed, isotropic etching occurs. (c) Several passivation and etch cycles are performed to obtain a directional etch. The passivation end point is set by the removal of the passivation layer $\left(b_{1}\right)$ and determines profile taper.

\subsection{Determination of fabrication criteria}

The pattern transfer into the ML inherently results in artifacts, such as sidewall scalloping, that were not considered in the LMG single-order derivation [5, 6] (see also section 3.3). In order to understand the effects of such artifacts, we first briefly recall the operating principle of single-order LMGs. The bandwidth of conventional ML mirrors is determined by the number of bi-layers effectively $\left(N_{e f f}\right)$ contributing to the reflection. However, $N_{\text {eff }}$ is limited due to the high absorption in the EUV and SXR spectral ranges. A reduction in absorption can be achieved by reducing the effective density, i.e. the lateral average per bilayer, by fabricating a grating structure into the ML mirror. When operating in 


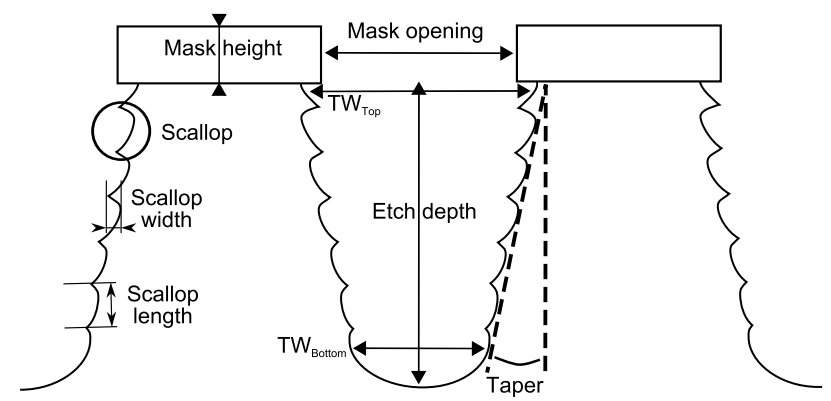

Figure 4.2: Schematic of definitions used to characterize etched nanostructures. Etch rates $(E R)$ are determined by dividing the etch depth $(E D)$ by the etch time. The trench aspect-ratio $\left(A R_{\text {trench }}\right)$ is given by dividing the depth in the trench by the mask opening. Scallops are characterized by their width $a$, length $l$ and ratio $a / l$. Profile taper is the angle of the sidewall and is determined from the top $\left(T W_{t o p}\right)$ and bottom $\left(T W_{\text {bottom }}\right)$ trench widths and $E D$.

the single-order regime, such gratings do not suffer from diffraction losses. The optical performance, in terms of bandwidth and peak reflectivity, of LMGs is then largely determined by the lamel-width-to-grating-period ratio $\Gamma$ (see figure 3.1) which simply indicates the reduction in effective density per bilayer [5-7]. Compared to a standard ML mirror, the bandwidth of an LMG is reduced by a factor $1 / \Gamma$, whereas the number of bi-layers should be increased by the same factor to ensure the peak reflectivity remains as high as for a conventional ML mirror. In this derivation, a constant effective density in the depth of the ML was assumed. However, artifacts such as taper and scalloping can introduce a depth dependence of the effective density. This was analyzed using a more generally valid version of our CWA.

\subsubsection{Generalized Coupled Waves Approach}

For the derivation of generalized Coupled Waves Approach (CWA) equations, we assumed a non-ideal lamel profile with mirror-inverted sidewalls defined by the function $p(z)$, i.e. if the right sidewall is defined by $p(z)$, then the left sidewall is defined by $-p(z)$. We also assumed that the lamel width $2 p(z)$ does not exceed the grating period $D$ at any $z$. The spatial distribution of the dielectric constant was then written as:

$$
\begin{gathered}
\epsilon(x, z<0)=1 ; \quad \epsilon(x, z>H)=1-\chi_{s u b} \\
\epsilon(x, 0 \leq z \leq H)=1-\chi(z) U(x, z)
\end{gathered}
$$

where $\chi(z)$ is the susceptibility of a multilayer structure varying with the depth and $\chi_{s u b}$ is the constant susceptibility of the substrate. The function 
$U(x, z)=1$, if the point $(x, z)$ is placed inside the multilayer structure and $U=0$ otherwise.

Assuming the dielectric constant to be a periodic function of $x$, we represented the function $U$ as the Fourier series:

$$
U(x, z)=\sum_{n=-\infty}^{+\infty} U_{n}(z) e^{2 i \pi n x / D}
$$

where

$$
U_{n}(z)=\frac{1}{\pi n} \sin \left(\frac{2 \pi n p(z)}{D}\right)
$$

with $U_{0}=2 p(z) / D$. Please note as in section 3.2.1 that eq. (4.3) differs to that used in our previous works $[5,6]$ as the point $x=0$ was now chosen to be in the center of the lamel instead of at the left side of the lamel. Shifting the coordinates of the previous works by $x^{\prime}=x-\Gamma D / 2$ yields the same coefficients as used in this work.

Following the steps also used in ref. [5] and section 3.2, we eventually obtained an infinite system of coupled waves equations describing the amplitudes $F$ of incident and reflected waves:

$$
F_{n}^{\prime \prime}(z)+\kappa_{n}^{2} F_{n}(Z)=k^{2} \sum_{m} \chi(z) U_{n-m}(z) F_{m}(z)
$$

for $n=0, \pm 1, \pm 2, \ldots$ with boundary conditions:

$$
F_{n}^{\prime}(0)+i \kappa_{n} F_{n}(0)=2 i \kappa_{n} \delta_{n, 0} ; \quad F_{n}^{\prime}(L)-i \kappa_{n}^{(s)} F_{n}(L)=0
$$

where $\kappa_{n}=\sqrt{k^{2}-q_{n}^{2}}$ and $\kappa_{n}^{(s)}=\sqrt{k^{2} \epsilon_{s u b}-q_{n}^{2}}$ are the Z-components of the wave vectors for the $n^{\text {th }}$ diffraction order in vacuum and the substrate, respectively, and $\delta_{n, 0}$ is the Kronecker symbol. The system (4.4)-(4.5) can be used to simulate the optical effects of non-ideal lamel profiles, including taper and scalloping, by numerically solving this system as previously done in ref. [5] and section 3.2.3.

For illustrative calculations in this work, we considered LMGs based on W/Si ML structures with parameters corresponding to those used in our experiments. The densities were taken to be $\rho_{W}=19 \mathrm{~g} / \mathrm{cm}^{3}$ and $\rho_{S i}=2.35 \mathrm{~g} / \mathrm{cm}^{3}$, bi-layer period $d=2.53 \mathrm{~nm}$, absorber-to-bi-layer thickness ratio $\gamma=0.28$ and with $N=400$ bi-layers. Interfacial roughness was not taken into account in these calculations. The parameters of the grating were chosen to be a period of $D=300 \mathrm{~nm}$ with a lamel-width-to-grating-period ratio $\Gamma=0.25$ to ensure single-order operation.

\subsubsection{Optical effect of tapering}

To analyze the effect of a tapered lamel profile, we used the trapezoidal function $p(z)=D \Gamma_{\text {top }} / 2+D\left(\Gamma_{\text {bottom }}-\Gamma_{\text {top }}\right) z /(2 H)$ as shown in figure 4.3 a to describe 


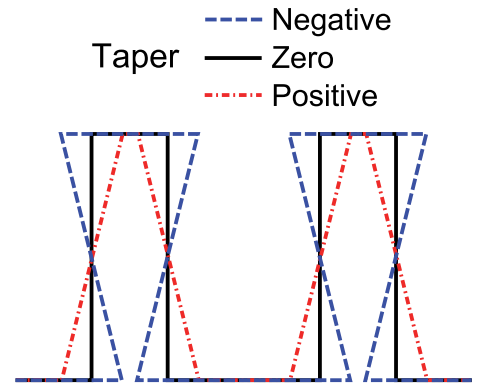

(a)

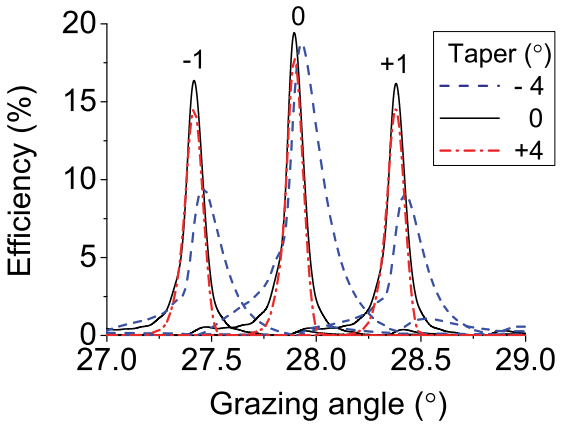

(b)

Figure 4.3: (a) Schematic of various lamel profiles. (b) Simulations of $\mathrm{W} / \mathrm{Si}$ LMG reflectance at an incident energy of $525 \mathrm{eV}$ for a rectangular lamel shape (solid black, $\Gamma=0.25$ ) and trapezoidal lamel shapes with the same average $\Gamma$-ratio, but with a negative (red dashed, $\Gamma_{t o p}=0.49, \Gamma_{\text {bottom }}=0.01$ ) or positive (blue dashed-dotted, $\Gamma_{t o p}=0.01, \Gamma_{\text {bottom }}=0.49$ ) taper.

the sidewall profile. The effect of various tapers on LMG reflection were then simulated assuming the LMG as described above and keeping the average $\Gamma$ ratio in the depth of the lamel constant at 0.25 . Results of these calculations are shown in figure $4.3 \mathrm{~b}$, where it can easily be seen that the effect of taper can actually be significant, especially for negative tapers. The largest change was found for the unrealistic taper of $-4.3^{\circ}$, i.e. for a lamel width of $0 \mathrm{~nm}$ at the bottom of the structure. The $0^{\text {th }}$ order bandwidth is then almost doubled from 1.7 to $3.0 \mathrm{eV}$ and the peak reflectivity was slightly reduced from 19.7 to $18.8 \%$. For a positive taper of the same absolute value, the bandwidth is slightly narrower at $1.6 \mathrm{eV}$ and the peak reflectivity is reduced to $17.9 \%$. Furthermore, the positive taper showed increased background suppression at angles just outside the main Bragg peak.

The difference in effects of a positive and negative taper can be explained by considering the effect of taper as a change in effective density, and hence absorption, in the depth of the ML. For a positive taper the density is reduced near the top of the structure, which results in a larger contribution of reflections from lower bi-layers to the interference and thereby resulting in a bandwidth reduction. On the other hand, a negative taper results in increased density near the top of the structure and actually limits the contribution of reflections from lower bi-layers to the interference resulting in increased bandwidths. As the average $\Gamma$-ratio is constant, the effect on $0^{\text {th }}$ order peak efficiency is limited.

Finally, it is important to recall that the $\Gamma$-ratio largely determines LMG optical performance (see also section 3.3). Accurate control of this ratio is hence required to achieve the designed optical performance. For a low taper process, such control can easily be obtained by simply varying the mask dimensions. 
Hence, to ensure tapering does not affect the optical performance of LMGs, tapers should be limited to below $\pm 1^{\circ}$, where slight positive tapers are preferred over negative ones.

\subsubsection{Optical effect of scalloping}

To analyze the effect of scalloping, the sidewall profile was described by the periodic function $p(z)=\Gamma D / 2+a / 2 \cdot \sin (2 \pi z / l)$. For single-order LMG operation, the effective susceptibility $\chi_{\text {eff }}(z)=\chi(z) \cdot 2 p(z) / D$ is given by the product of two periodic functions with periods $d$ and $l$. If the ratio $d / l$ is an irrational number, the function $\chi_{e f f}(z)$ is not periodic. Expanding the functions $\chi(z)$ and $p(z)$ into the Fourier series, we found that $\chi_{\text {eff }}(z)$ contains terms with four main periods $d_{j}(j=0-3)$, namely $d_{0}=d, d_{1}=l, d_{2}=d /(1+d / l)$ and $d_{3}=d /(1-d / l)$ as well as higher harmonics with periods $d_{j} / n(n=2,3, \ldots)$. As the scallop period $l$ is typically much larger than the ML mirror period $d_{0}$, reflection from this scallop period was not considered. However, the periods $d_{2}$ and $d_{3}$ may be close to the ML mirror period $d_{0}$ if the scallop length $l$ is large enough. A similar layered structure containing terms with different periods was analyzed in details in ref. [81] as applied to the problem of simultaneous reflection of two arbitrary (not multiple) wavelengths from a multilayer mirror.

As for single-order operation (section 3.3), only the first order Bragg reflection is of interest and higher order terms in the Fourier series are neglected. The depth-distribution of the effective susceptibility is then written in the simplified form:

$$
\begin{gathered}
\chi_{e f f}(z) \approx \Gamma \bar{\chi}+\frac{a}{D} \bar{\chi} \sin \left(\frac{2 \pi z}{l}\right)+\left(\chi_{A}-\chi_{S}\right) \frac{\sin (\pi \gamma)}{\pi} . \\
{\left[2 \Gamma \cos \left(\frac{2 \pi z}{d_{0}}-\pi \gamma\right)+\frac{a}{D} \sin \left(\frac{2 \pi z}{d_{2}}-\pi \gamma\right)-\frac{a}{D} \sin \left(\frac{2 \pi z}{d_{3}}-\pi \gamma\right)\right]}
\end{gathered}
$$

If the grazing angle of the incident beam is varied, resonant Bragg reflection is observed from each of the three periods such that $\lambda=2 d_{j} \sin \left(\Theta_{m=0}^{(j)}\right)$, where $m=0$ indicates the $0^{t h}$ diffraction order and $j=0,2,3$ is the index of the period. The peak from $j=0$ is the Bragg order, whereas $j=$ 2,3 are peaks caused by scalloping and are referred to as scallop harmonics. Similarly, three diffraction peaks are also seen for any $m^{\text {th }}$ order such that $\lambda=2 d_{j}\left(\sin \left(\Theta_{m}^{(j)}\right)+\sin \left(\Phi_{m}^{(j)}\right)\right)$, where the periods $d_{j}$ are different and the diffraction angle $\Phi_{m}^{(j)}$, i.e. the grazing angle of the outgoing diffracted beam, is determined by the grazing incidence angle via the grating equation $\lambda=D\left(\cos \left(\Theta_{m}^{(j)}\right)-\cos \left(\Phi_{m}^{(j)}\right)\right)$. The optical effect of scalloping can therefore be considered as the appearance of a phase grating in the depth of the ML structure, which can be described by a number of embedded diffraction gratings with the same $D$ and $\Gamma$, but different ML periods $d_{j}$. The angle $\Theta_{m}^{(j)}$ can be referred to as the quasi-Bragg resonance angle and is comparable to the 
blaze angle in the theory of conventional grazing incidence diffraction gratings.

In general, the effect of scallops on the main reflectivity peak is expected to be very small. First of all eq. (4.6) shows that the amplitude of the Bragg order from period $d_{0}$ is independent of the scallop width $a$ and, secondly, the scallop width $a$ is typically $<<2 \Gamma D$ resulting in small amplitudes of the scallop harmonics from periods $d_{2}$ and $d_{3}$. The coupling of waves diffracted from the different gratings is also typically weak, except for the specific case when the Bragg angle of reflection from the ML with period $d_{0}$ coincides with the angle of a scallop harmonic from the gratings with ML period $d_{2}$ or $d_{3}$, i.e. when $\Theta_{0}^{(0)}=\Phi_{+m}^{(2)}$ or $\Theta_{0}^{(0)}=\Phi_{-m}^{(3)}$. In these cases the incident wave effectively excites at least two waves, namely the specularly reflected wave and the scallop harmonic wave from the higher diffraction order. As two waves can be excited, the LMG is actually no longer in single-order operation even for LMGs that do fulfill the single-order condition (eq. (3.39). This results in a loss of peak efficiency as not all incident energy is reflected and diffracted into a single order. To determine the conditions for when this effect occurs, simultaneous resonant excitation of the specularly reflected and, for definiteness, $+1^{\text {st }}$ order diffracted waves (i.e. the case when $\left.\Theta_{0}^{(0)}=\Phi_{+1}^{(2)}\right)$ were examined. This resulted in the following system of equations:

$$
\begin{aligned}
& \lambda=2 d_{0} \sin \left(\Theta_{0}^{(0)}\right) \\
& \lambda=d_{2}\left(\sin \left(\Theta_{0}^{(0)}\right)+\sin \left(\Phi_{+1}^{(2)}\right)\right) \\
& \lambda=D\left(\cos \left(\Theta_{0}^{(0)}\right)-\cos \left(\Phi_{+1}^{(2)}\right)\right)
\end{aligned}
$$

Taking into account that $\lambda<<D$, i.e. $\Phi_{+1}^{(2)}$ is close to $\Theta_{0}^{(0)}$, and expanding $\sin \left(\Phi_{+1}^{(2)}\right)$ and $\cos \left(\Phi_{+1}^{(2)}\right)$ in a series near $\Theta_{0}^{(0)}$, the system (4.7) is fulfilled if the scallop length obeys the following condition:

$$
l_{\text {worst }} \approx D \tan \left(\Theta_{0}^{(0)}\right) \text {, }
$$

where $l_{\text {worst }}$ indicates the worst scallop length, i.e. the scallop length for which the $0^{\text {th }}$ order peak reflectivity is reduced the most. The same derivation can also be performed for Bragg orders arising from period $d_{3}$, which gives the same result. A similar condition can also be written for higher diffraction order efficiencies by replacing $\Theta_{0}^{(0)}$ with $\Theta_{m}^{(0)}$.

The above results are demonstrated by figure 4.4 where the $0^{\text {th }}$ and $\pm 1^{\text {st }}$ orders are shown for various scallop lengths and widths. From figure 4.4b, it can easily be seen that the scalloping results in additional Bragg peaks, the scallop harmonics, as indicated by the red arrows. Figure $4.4 \mathrm{a}$ then demonstrates that at the worst scallop length of $160 \mathrm{~nm}$ for this particular structure, as predicted by eq. (4.8), there is indeed a significant overlap of the scallop harmonics with the higher diffraction orders. This overlap results in a strong decrease of peak reflectivity. 


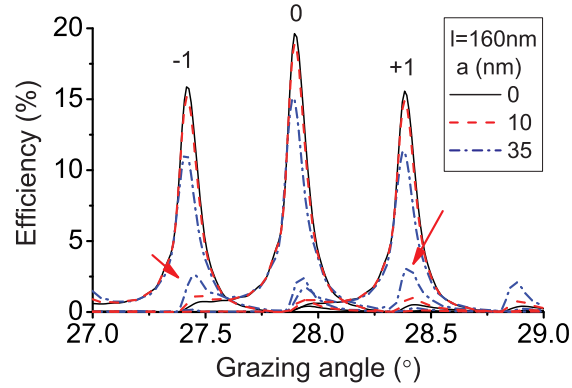

(a)

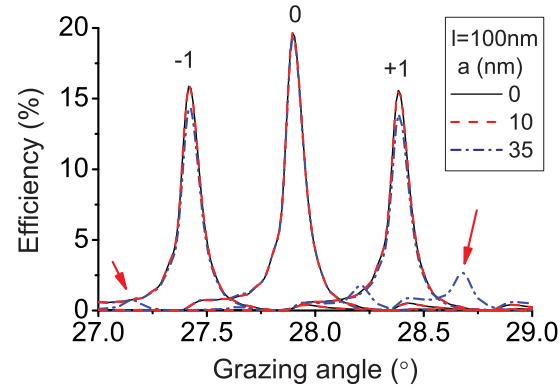

(b)

Figure 4.4: W/Si LMG reflectance at the incident energy of $525 \mathrm{eV}$ for various scallop widths (a) at the worst scallop length $\left(l_{\text {worst }}\right)$ of $160 \mathrm{~nm}$ predicted by eq. (4.8) and (b) at a scallop length of $l=100 \mathrm{~nm}$. Additional Bragg orders due to scalloping, the scallop harmonics, are indicated by the red arrows.

Evidently, the appearance of these scallop harmonics of considerable height is unacceptable for the application of LMGs in SXR spectroscopic applications as they may render analysis of emission spectra very difficult or even impossible. The effect of scalloping can be limited by ensuring that the scallop length is sufficiently far from $l_{\text {worst }}$, as shown by figure 4.4 b, or by reducing the scallop width $a$ to below $10 \mathrm{~nm}$, as shown by the $a=10 \mathrm{~nm}$ curve in figure $4.4 \mathrm{a}$. For both cases the difference in peak reflectivity with the curve assuming no scalloping is less than $1 \mathrm{pp}$. In general, the fabrication process should therefore result in scallop widths as small as possible, at least below $10 \mathrm{~nm}$, while the scallop lengths should not equal $l_{\text {worst }}$.

\subsubsection{Fabrication criteria for W/Si LMGs}

To determine fabrication criteria, we also investigated the effect of an LMG simultaneously suffering from both scalloping and taper. For this analysis, values of taper and scalloping were assumed that were within the limits derived in the previous sections, i.e. $+1^{\circ}$ taper and scallops with lengths of $40 \mathrm{~nm}$ and widths of $10 \mathrm{~nm}$. The simulations showed that no additional deterioration of W/Si LMG optical performance was to be expected from LMG suffering from such combined artifacts.

This allowed us to determine fabrication criteria for W/Si LMGs as summarized in table 4.1. Here, the selectivity (ratio of ML-to-mask etch rates) was also included, which is required to enable the etching of $1 \mu \mathrm{m}$ ML stacks with the mask used in this work. 
Table 4.1: Fabrication criteria for W/Si LMGs

\begin{tabular}{c|c|c} 
Etch result & Specification & Motivation \\
\hline Etch depth & $\geq 1 \mu \mathrm{m}$ & ML stack height \\
Selectivity & $\leq 10: 1$ & Mask height $\sim 130 \mathrm{~nm}$ \\
Taper & $\leq \pm 1^{\circ}$ & Dimension control \\
Scallop length & $\neq D \tan \left(\Theta_{0}^{(0)}\right)$ & Overlap of harmonics \\
Scallop width & $\leq 10 \mathrm{~nm}$ & Harmonic amplitude \\
Grating dependence & Independent & Varying feature sizes
\end{tabular}

\subsection{Etching of $\mathrm{W} / \mathrm{Si}$ ML mirrors}

For the actual fabrication of LMGs in ML mirrors, we first determined the process window by investigating the fabrication of silicon nanostructures. Next, multilayer etching was compared with silicon etching and the control of scalloping, taper and grating structure dependence when etching MLs was investigated.

\subsubsection{Etch settings}

The process window for the fabrication of nanostructures in ML mirrors, as stated in table 4.2, was determined from an investigation into silicon etching and previous works [75, 82]. For the fabrication of nanostructures, low etch rates of at most a few hundred $\mathrm{nm} / \mathrm{min}$ are required, which was achieved by using low ICP powers, low gas-flow rates and moving the sample as far from the plasma as possible. To ensure maximum directionality, the Ion Angular Distribution (IAD) was minimized by fully opening the pressure control valve (APC) to keep the pressure as low as possible, which was typically in the $10^{-3}-10^{-2}$ mbar range. CCP was initially increased to ensure it would not be limiting during etch experiments. Although temperatures down to $-120^{\circ} \mathrm{C}$ were investigated, values above $-50^{\circ} \mathrm{C}$ were required to etch tungsten at a reasonable rate $[83,84]$.

Table 4.2: Process window used for the study of etching nanoscale optical structures in ML mirrors. The APC was fully opened to minimize pressure and maximize directionality. An offset of $-100 \mathrm{sccm}$ in $\mathrm{SF}_{6}$-flow and $-150 \mathrm{sccm}$ in $\mathrm{C}_{4} \mathrm{~F}_{8}$-flow were found at a later time.

\begin{tabular}{c|c|c|c|c|c|c|c|c|c|c|c|c} 
& \multicolumn{3}{|c|}{$\mathrm{SF}_{6}$} & \multicolumn{4}{|c|}{$\mathrm{C}_{4} \mathrm{~F}_{8}$} & $\mathrm{ICP}$ & \multicolumn{2}{c|}{$\mathrm{CCP}$} & $\mathrm{SH}$ & $\mathrm{T}$ \\
& Prio & Flow & $\mathrm{t}$ & Prio & Flow & $\mathrm{t}$ & $\mathrm{P}$ & $\mathrm{P}$ & on & off & - & - \\
& - & sccm & $\mathrm{s}$ & - & $\mathrm{sccm}$ & $\mathrm{s}$ & $\mathrm{W}$ & $\mathrm{W}$ & $\mathrm{ms}$ & $\mathrm{ms}$ & $\mathrm{mm}$ & ${ }^{\circ} \mathrm{C}$ \\
\hline $\min$ & 2 & 10 & 1 & 1 & 20 & 0.5 & 300 & 20 & 10 & 90 & 110 & -120 \\
$\max$ & 2 & 150 & 6 & 1 & 200 & 4 & 1500 & 110 & 25 & 75 & 200 & +20
\end{tabular}




\subsubsection{Comparison of ML with silicon etching}

Due to the thin-layered structure with dual-material composition of ML mirrors, the etching significantly differs from pure silicon structures. This is demonstrated by figure 4.5 which shows two simultaneously etched structures in a silicon and ML substrate, respectively. As can be seen, the ML etches significantly slower than the silicon and exhibits a positive taper whereas the silicon substrate shows a negative taper. The ML also appears to have significantly less scalloping.

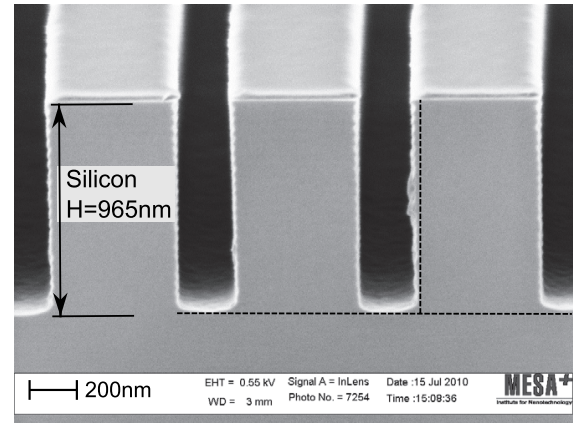

(a)

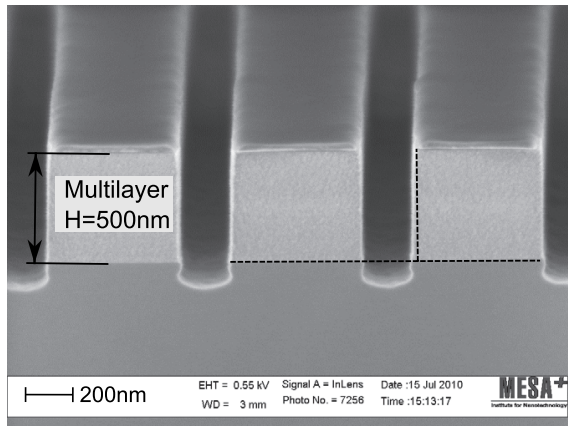

(b)

Figure 4.5: SEM images of two structures etched using identical settings into (a) a silicon substrate and (b) a $500 \mathrm{~nm}$ W/Si ML structure. The dashed lines are guides to the eye to clarify the difference in taper, which is slightly negative for silicon and slightly positive for the ML.

To investigate these differences in more detail, we compared the etching of pure silicon and tungsten. The standard enthalpy of formation $\left(\Delta_{f} H_{m}^{0}\right)$ for $\mathrm{WF}_{6}$ is $-1721 \mathrm{~kJ} \mathrm{~mol}^{-1}$ and $-1615 \mathrm{~kJ} \mathrm{~mol}^{-1}$ for $\mathrm{SiF}_{4}[76,85]$, indicating both tungsten and silicon can be etched with fluorine radicals. Next, the chemical volatility of the ML materials with fluorine was determined using the activation energy $E_{a c t}$, where a low activation energy indicates a high volatility. $E_{a c t}$ can be determined from the Arrhenius equation:

$$
E R=E R_{\text {sat }} \cdot e^{-q E_{a c t} /(k T)},
$$

where $E R_{\text {sat }}$ is the saturated etch rate at high temperature, $q$ is the electron charge, $k$ is the Boltzmann constant and $T$ is the temperature [75].

To experimentally determine the activation energy of the reaction with fluorine, silicon and ML etch rates were measured for $50 \mu \mathrm{m}$ half pitch gratings using only the etch cycle of the Bosch process. The samples were diced and placed on a carrier wafer as described in section 4.2 . < $100>$ p-type silicon wafers were used for silicon samples and $100 \mathrm{~nm}$ thick tungsten samples were 
prepared via argon sputtering deposition. Etch depths were determined using stylus profilometry. The resulting Arrhenius plots are shown in figure 4.6 and clearly indicate the difference in volatility with fluorine between silicon and tungsten. Where silicon has an $E_{a c t}$ of only $2 \mathrm{meV}$, meaning this reaction can be considered spontaneous [75], $E_{a c t}$ of tungsten was determined to be $60 \mathrm{meV}[84,86]$. As the thermal energy in the temperature range used is between 13 and $25 \mathrm{meV}$, additional energy is required to initiate the etching of tungsten. This demonstrates that although tungsten does react chemically with fluorine radicals, the reaction will be slower than for silicon. Based on the volatility and activation energy of the reaction with fluorine and the absorber layer thickness, other ML materials can be determined that can be etched with the process described here.

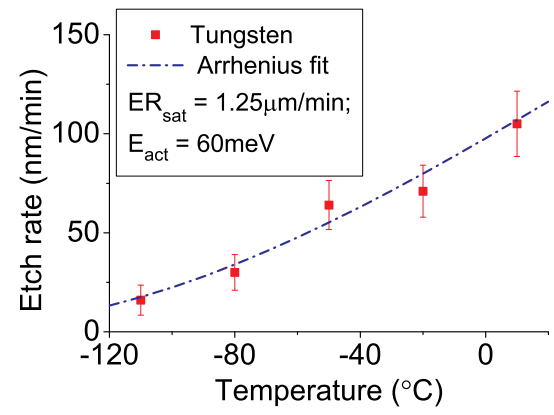

(a)

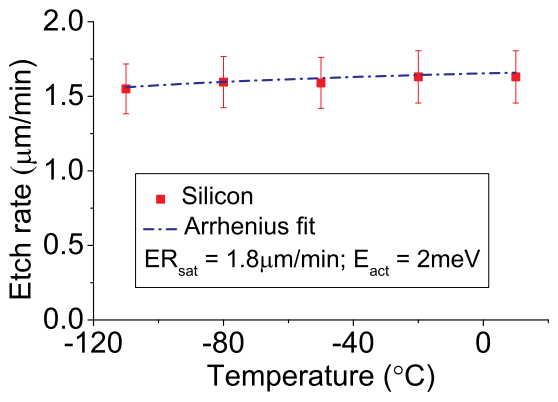

(b)

Figure 4.6: Arrhenius plots of (a) tungsten and (b) silicon etching. Please note the difference in units of the etch rates which is $\mathrm{nm} / \mathrm{min}$ for tungsten, but $\mu \mathrm{m} / \mathrm{min}$ for silicon.

Based on the silicon and tungsten etch rates of figure 4.6, we determined an expected etch rate for the W/Si ML based on the etch time per layer using:

$$
E R_{M L}=\frac{d}{\frac{\gamma d}{E R_{W}}+\frac{(1-\gamma) d}{E R_{S i}}} .
$$

This expected ML etch rate was compared to the actual measured etch rate in figure 4.7, where it is easily seen that the ML etches much faster than predicted by eq. (4.10). This discrepancy can be explained by the formation of $\mathrm{WSi}_{x}$ compounds in the tungsten layer. From ref. [87], it is known that typical interlayer thicknesses of $\mathrm{WSi}_{x}$ compounds exceeds our high- $Z$ layer thickness, meaning the entire tungsten layer is expected to consist of silicides.

Such silicides still react with fluorine as the standard enthalpies of formation $\left(\Delta_{f} H_{m}^{0}\right)$ for the two main tungsten silicides $\left(-82 \pm 6 \mathrm{~kJ} \mathrm{~mol}^{-1}\right.$ for $\mathrm{WSi}_{2}$ and $-133 \pm 25$ for $\left.\mathrm{W}_{5} \mathrm{Si}_{3}[88,89]\right)$ are much higher than for the fluorine compounds. It is also known from previous works, although based on a F-plasma from $\mathrm{CF}_{4} / \mathrm{O}_{2}$, that such silicides actually etch at higher rates than the pure 
metal, meaning $E_{\text {act }}$ will probably be between the tungsten and silicon values [90, 91]. However, additional reactions can also occur in such dual-material structures. The most important of which is the reduction of $\mathrm{WF}_{6}$ in the presence of silicon [85]:

$$
2 W F_{6}+3 S i \rightarrow 2 W+3 S_{i F},
$$

resulting in a $\Delta_{f} H$ of $-1401.6 \mathrm{~kJ} \mathrm{~mol}^{-1}$. Due to the close proximity of silicon, it is not expected that this reaction results in redeposition of tungsten over a wide area. However, an immediate redeposition at the sidewalls would be possible, resulting in a delay of tungsten etching.

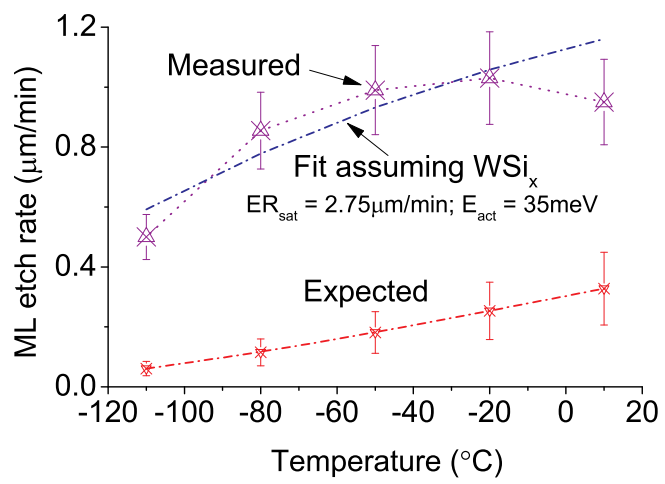

Figure 4.7: Comparison of expected and measured ML etch rates as function of temperature. The measured ML etch rate was higher than expected based on the independent silicon and tungsten etch rates, which is probably due to tungsten silicide formation.

To determine if compound formation explains the difference in measured and expected ML etch rates, values of $E R_{\text {sat }}$ and $E_{a c t}$ for $\mathrm{WSi}_{x}$ were fitted to the measured ML etch rates using eq. (4.10). As can be seen, the trend of the fit differs from the measured ML etch rates. It is suspected that this discrepancy is caused by the profilometric determination of the etch rates, which is affected by the anisotropy ratio, i.e. lateral-to-directional etch ratio. From previous works [91], it is known that $\mathrm{Si}, \mathrm{W}$ and $\mathrm{WSi}_{2}$ have different temperature dependencies of the anisotropy ratios when etched using $\mathrm{SF}_{6}$ plasmas. Most notably, $\mathrm{WSi}_{2}$ showed a clear reduction in the anisotropy ratio at temperatures above $\sim-40^{\circ} \mathrm{C}$, whereas the anisotropy ratio of pure silicon and tungsten remained constant in the temperature range used. Having determined the difference between etching of silicon and tungsten, we investigated the effect of the ML structure on scalloping, taper and grating dependence. 


\subsubsection{Control of scallop formation}

The dimensions of the typical scalloping in Bosch DRIE for silicon structures are mostly determined by the etch rates and duration of the etch cycle. The etch rate depends on the amount of fluorine radicals in the plasma, which is determined by the $\mathrm{SF}_{6}$-flow and ICP-power. The scallop-ratio in silicon is generally 0.3 and decreases with trench aspect-ratio [91, 92]. However, the scallop formation in ML structures is quite different to that in silicon as can be seen in figure 4.8a. Here, a Transmission Electron Microscope (TEM) image is shown of which the top part is a ML and the bottom is silicon. It can easily be seen that the scallops in silicon are much larger than in the ML.

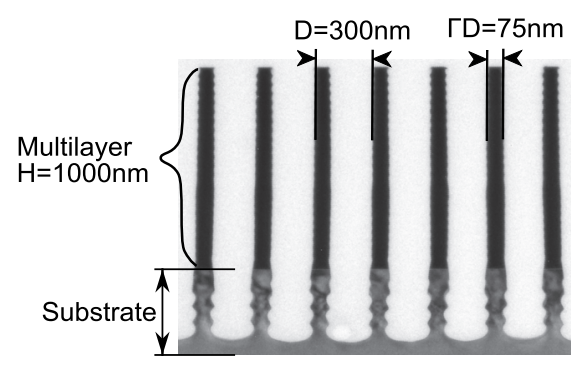

(a)

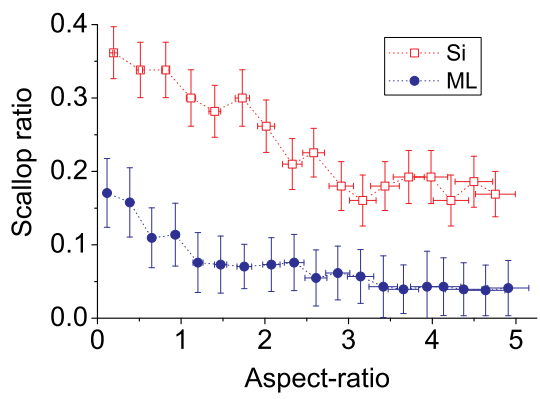

(b)

Figure 4.8: (a) TEM image of an LMG with a grating period $D$ of $300 \mathrm{~nm}$ and a lamel width $\Gamma D$ of $75 \mathrm{~nm}$ etched into a $1 \mu \mathrm{m}$ high $\mathrm{W} / \mathrm{Si}$ ML stack. (b) Comparison of scallop ratio (width-to-length) between figure (a) and a similar structure etched into pure silicon using the same settings.

As this difference could be caused by the reduction in etch rate of ML, we also compared the scallop ratios. The scallop-ratio in a ML and silicon substrate are shown as function of trench aspect-ratio in figure $4.8 \mathrm{~b}$, where it can be seen that the scallop-ratio in the ML structure is suppressed by a factor of more than 2 with respect to the silicon structure. This reduction is caused by the tungsten layers in the ML structure. For silicon structures, the scallop width is mostly determined by the isotropic chemical etching. However, the tungsten etching is much less spontaneous than silicon etching and therefore results in less isotropic etching. Specifically, laterally moving species have limited additional kinetic energy and are therefore not able to initiate the tungsten etching in those directions. As the silicon layers are relatively thin $(\approx 1-1.5 \mathrm{~nm})$, exchange of etch species within only this layer is insufficient to sustain the isotropic profile of silicon etching in the ML structure.

Although the silicon process already resulted in scallop lengths that were sufficiently small compared to the fabrication criteria, the scallop widths were too large. However, the same process in the ML resulted in acceptable scallop 
dimensions as etch rates were reduced and scallop formation was suppressed. Scallop lengths below $50 \mathrm{~nm}$ and widths below $10 \mathrm{~nm}$ were thus easily achieved in the ML structure. Near the bottom of the structure, the scallop width was even reduced to below the SEM resolution of approximately $2 \mathrm{~nm}$. We used the scallop suppression by the ML structure to obtain low tapers with minimal grating structure dependence as demonstrated in the following sections.

\subsubsection{Minimizing profile taper}

In the Bosch DRIE process, profile taper is mainly determined by the balance between passivation layer deposition and removal as this shifts the passivation end point (figure 4.1) and therefore affects the position of the following isotropic step. Taper can be altered by the changing the passivation layer deposition by varying the ICP power or temperature. However, ICP significantly affects the composition of the plasma and should therefore not be changed. The temperature can also not be varied over a large range as the tungsten layer becomes increasingly difficult to etch at lower temperatures. The passivation layer removal is mainly affected by the CCP, IAD and etch cycle time [75]. As previously stated, the CCP should be minimized in order to achieve maximum selectivity and the IAD should be maximally directional by reducing the pressure. By using sufficiently long cycle times, the IAD determines the passivation end point and, hence, controls taper. Although longer cycle times also result in increased scalloping, the scallop suppression of the ML structure prevents scallops that affect LMG optical performance.

Comparing the resulting taper of an identical process in a ML to a silicon structure, it can clearly be seen in figure 4.9 that the ML structure increased taper by roughly one degree. This is because the taper in the silicon process is largely determined by the IAD as the silicon etch is spontaneous. However, the etching of tungsten requires additional energy to be initiated and the Ion Energy Distribution (IED) therefore also affects taper in a ML structure. Similar to the scallop suppression argument, it can be understood that highly directional species have the highest kinetic energy, whereas laterally moving species only have limited additional kinetic energy. Although the ML is then exposed to the same IAD as the silicon process, the tungsten layer actually shifts the effective passivation end point slightly inward. Here, effective indicates the fact that the actual passivation layer can be completely removed and that the tungsten layer partially takes over the role of the passivation layer as far as the end point is concerned. For the same reason, etching of a ML structure actually also increases reproducibility as can be seen in the lower residual tapers, i.e. difference between measured taper and linear fit of figure $4.9 \mathrm{a}$, as shown in figure 4.9b. 


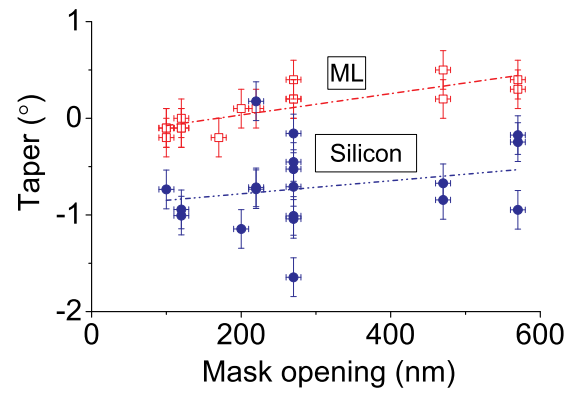

(a)

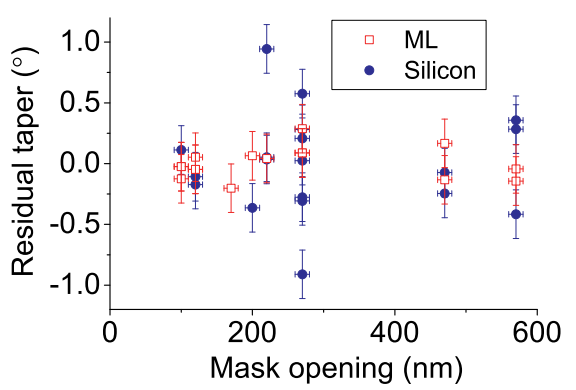

(b)

Figure 4.9: (a) Comparison of taper in ML and silicon substrates etched using the same process. The lines are linear fits to guide the eye. (b) Comparison of residual tapers, i.e. the difference between the measured taper and the linear fit in (a), for both ML and silicon structures.

\subsubsection{Reduction of grating structure dependence}

Besides the grating structure dependence of the taper, etch rates should also not differ too much for varying feature dimensions. Figure 4.10a shows that the ML etch rate increases by roughly $30 \%$ when increasing the mask opening from 100 to $700 \mathrm{~nm}$. Although the relative increase is comparable to that for silicon etching, the absolute effect is much smaller as the ML etch rate is lower. The figure also demonstrates the reproducibility of Bosch DRIE as several points in these figures were duplicate runs from which we determined etch rates to differ by less than $5 \%$ over a period of more than 1 year.

A major benefit of the limited grating structure dependence of tapers and etch rates is the resulting accuracy of the pattern transfer into the ML. The LMG $\Gamma$-ratio is easily controlled by simply varying the etch mask. A SEM image of a fabricated LMG with a $200 \mathrm{~nm}$ period is shown in figure 4.10b, which achieved a 17:1 lamel aspect-ratio due to the low tapering of the ML etching. The low taper and etch rate dependence on grating structure is not only beneficial for LMG fabrication, but becomes even more important when fabricating single structures with multiple feature dimensions such as zoneplates for the SXR or EUV spectral range.

\subsection{Conclusions}

We have demonstrated successful etching of multilayer (ML) mirrors for the fabrication of Lamellar Multilayer Gratings (LMGs). Using the developed process, LMGs with a grating period of only $200 \mathrm{~nm}$ and a lamel width of only $60 \mathrm{~nm}$ were fabricated in a ML mirror consisting of $400 \mathrm{~W} / \mathrm{Si}$ bi-layers with a 


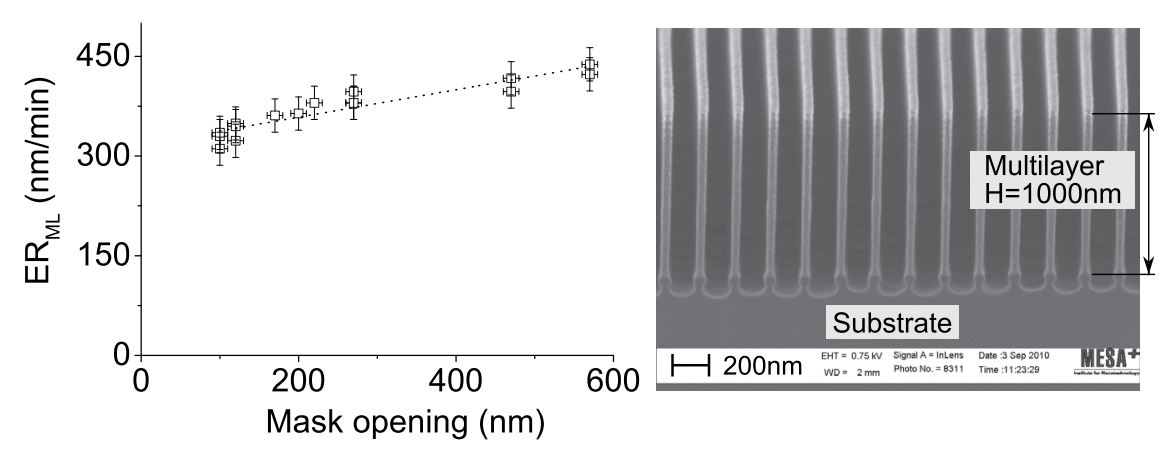

(a)

(b)

Figure 4.10: (a) ML etch rates $E R_{M L}$ and taper as function of trench opening. The line is a guide the eye. (b) SEM image of an LMG with a grating period $D$ of only $200 \mathrm{~nm}$ and a lamel width of $60 \mathrm{~nm}$, resulting in a lamel aspect ratio of 17:1.

$2.5 \mathrm{~nm}$ bi-layer period. The resulting lamel aspect-ratio was 17:1 as compared to previously reported values of $\sim 5: 1$ [71].

Etching of the ML was performed using Bosch Deep Reactive Ion Etching (DRIE), which provides directional etching, but also results in typical sidewall scalloping. Although this scalloping can significantly affect the optical performance of LMGs, this issue was negated by using the ML structure to suppress scallop formation. The suppression was determined to be caused by the activation energy of the high- $Z$ component, in our case tungsten, which was above thermal energy and hence required additional kinetic energy for the reaction to be initiated. As laterally moving species have less kinetic energy the etching of a ML is less isotropic than silicon, which does not require additional kinetic energy to be etched, and hence scallop formation is reduced. The scallop suppression also allowed for the fabrication of LMGs with low tapers and minimal grating structure dependence of the etch process. The minimal grating structure dependence is of importance for the fabrication of optical elements with varying feature dimensions, such as zoneplates.

By examining materials that react volatile with fluorine, other ML materials can be determined that can be etched using the same approach as presented in this work. Besides W/Si MLs, our approach can also be used to etch MLs with, amongst others, molybdenum or iridium as absorber and boron carbide or scandium as spacer. This means that our approach would also be very well suited to etch Mo/Si ML mirrors for EUV lithography or the Mo/B $\mathrm{B}_{4} \mathrm{ML}$ mirrors used for spectroscopy by refs. [12, 71]. However, it is important to realize that compound formation between absorber and spacer, the absorber layer thickness and the activation energy $E_{a c t}$ of the reaction between fluorine and the high- $Z$ material can significantly influence the actual etching process. 


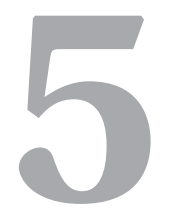

\section{Experimental demonstration of single-order LMG operation}

\subsection{Introduction}

In the previous chapters, we demonstrated optimal LMG performance to occur in the so-called single-order regime. Moreover, we determined a suitable fabrication method based on UV-NIL and Bosch DRIE. In this chapter, we report on the first experimental demonstration of single-order LMG operation in the SXR spectral range. Experimentally, single-order operation showed up as a lack of significant diffraction into higher orders, thereby increasing the peak reflectivity as compared to multi-order LMGs. Single-order operation also resulted in a improvement of spectral resolution $E / \Delta E$ of nearly four as compared with the corresponding unpatterned ML mirror.

We begin this chapter by comparing measured reflectivity curves from single- and multi-order LMGs in section 5.2. Next, in section 5.3 we investigate the single-order operation in more detail by studying the $\Gamma$-ratio dependence of the LMG performance. From this study, we determined that the initial multilayer limits LMG performance, which is discussed in more detail in section 5.4. Finally, in section 5.6 we present higher diffraction order measurements of single-order LMGs.

\subsection{Single versus multi-order operation}

To investigate whether single-order LMG operation can be obtained in the SXR spectral range, we fabricated a number of LMGs with various combinations of grating periods $(D)$, lamel widths $(\Gamma D)$ and $\Gamma$-ratios. The grating dimensions 
were chosen between 200 and $2000 \mathrm{~nm}$ with $\Gamma$-ratios between 0.2 and 1 . This enabled us to perform a systematic investigation of single- and multi-order operation as function of the grating parameters for SXR energies between 500 and $1500 \mathrm{eV}$. The LMGs were fabricated in a commercially obtained ML mirror [72], specified to be a 400 bi-layer W/Si ML with a bi-layer period $d$ of $2.53 \mathrm{~nm}$ and a $\gamma$ of 0.28 . SXR reflectivity spectra of these samples were measured at the PTB beamline at BESSYII, which achieves an energy precision below $0.1 \mathrm{eV}$ and a reflectance accuracy of $0.2 \%$ [45].

Figure 5.1 shows the measured absolute reflectivity of two LMGs with the same $\Gamma$-ratio of 0.4 at an SXR energy of $525 \mathrm{eV}$. From eq. (3.39) we derived that for single-order operation of these LMGs the lamel width $(\Gamma D)$ should be smaller than approximately $200 \mathrm{~nm}$, which corresponds to a grating period of $500 \mathrm{~nm}$ for a $\Gamma$ of 0.4 . The first spectrum (violet circles) is from a LMG with grating dimensions $D=1 \mu \mathrm{m}$ and $\Gamma D=400 \mathrm{~nm}$, while the second spectrum (blue squares) is from an LMG with a small grating period, namely $D=300 \mathrm{~nm}$ and $\Gamma D=120 \mathrm{~nm}$. The third trace (small red stars) is the reflectivity of the unpatterned ML mirror to provide a reference. This reference is slightly shifted in energy due to the higher average susceptibility of the individual layers as discussed in section 3.3.

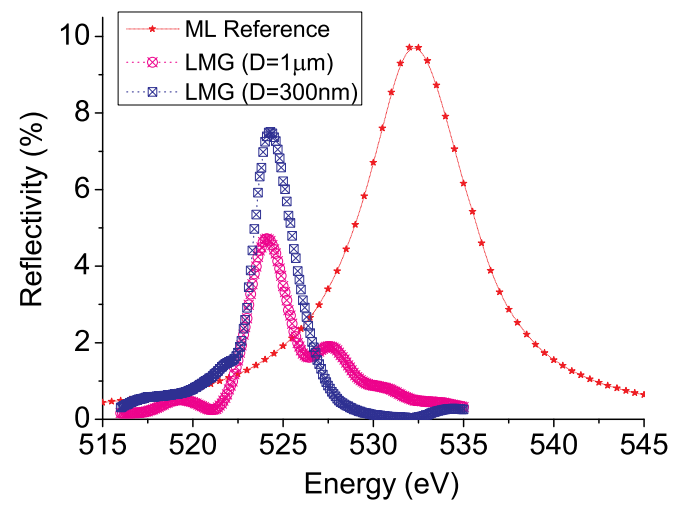

Figure 5.1: Experimental reflectivity for two LMGs with the same $\Gamma$ ratio of 0.4 , operating in the multi-order $(D=1 \mu \mathrm{m})$ and singleorder $(D=0.3 \mu \mathrm{m})$ regime. The reflectivity of an unpatterned ML mirror is also shown for reference. The lines are guides to the eye.

In the figure, it can be seen that the $D=300 \mathrm{~nm}$ LMG provides increased spectral resolution while this spectrum has no significant side maxima, in contrast to the $D=1 \mu \mathrm{m}$ LMG. At the same time, the $D=300 \mathrm{~nm}$ LMG also has a main Bragg peak which is about a factor of 1.5 higher than the main Bragg peak for the $D=1 \mu \mathrm{m}$ LMG. Both effects are due to the absence of significant diffraction into higher orders for the $D=300 \mathrm{~nm}$ LMG. Furthermore, the 
FWHM bandwidth of the $D=300 \mathrm{~nm}$ LMG is a factor of 2.5 narrower than that of the reference spectrum, which is in agreement with the $1 / \Gamma$ bandwidth scaling predicted for single-order operating LMGs in section 3.3 [5].

These observations prove that single-order LMG operation can be achieved in the SXR spectral range. The largest resolution improvement, also obtained with a single-order operating LMG around $525 \mathrm{eV}$, had a factor 3.8 narrower bandwidth $(1.85 \mathrm{eV})$ than that of the unpatterned ML mirror $(7.02 \mathrm{eV})$.

\section{$5.3 \quad$ Single-order $\Gamma$ scaling}

For a more systematic analysis of spectral narrowing in single-order LMG operation, we extended our experiments with reflectivity measurements at a higher photon energy $(E=1255 \mathrm{eV})$ where more LMGs can be operated in single-order for a broader range of $\Gamma$ values ( 0.2 to 0.8$)$. The measured spectral bandwidths and peak reflectivities are shown in figure 5.2 as a function of $\Gamma$. It can be seen in figure 5.2a that the spectral bandwidth of the LMG decreases with decreasing $\Gamma$, showing a factor 3.3 improvement in spectral resolution when going from the unpatterned ML mirror $(\Gamma=1)$ to an $\mathrm{LMG}$ with $\Gamma=0.22$. At the same time, it can be seen from figure $5.2 \mathrm{~b}$ that the peak reflectivity decreases for lower $\Gamma$-ratios and drops to approximately half of its initial value at $\Gamma=$ 0.2 .

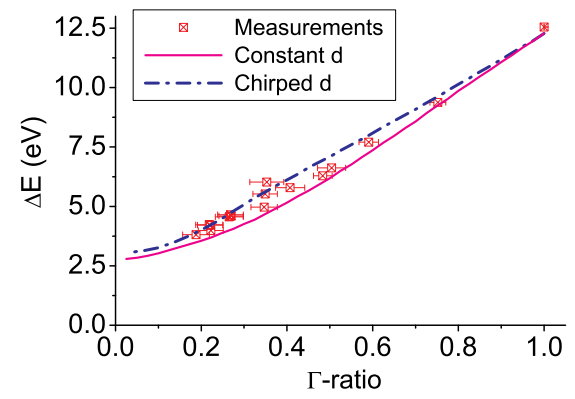

(a)

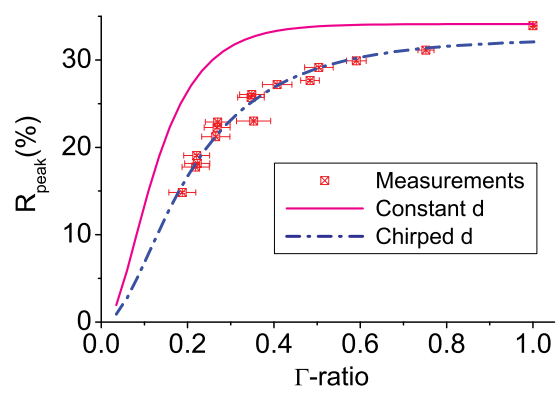

(b)

Figure 5.2: (a) FWHM and (b) peak reflectivity for several single-order LMGs at the SXR energy of $1255 \mathrm{eV}$. Results of calculations using our theoretical model including the measured bi-layer chirp are in good agreement with the measured data.

We compared the experimental data with theoretical calculations of the spectral bandwidths and peak reflectivities as function of $\Gamma$ using our Coupled Waves Approach (CWA) as derived in section 3.2. In these calculations, we included various effects usually found in ML mirrors, such as rms interlayer roughness $(\sigma)$ and interface width $(w)$. By fitting the calculations to the mea- 
sured spectra of the unpatterned ML mirror recorded at various energies and taking the finite number of bi-layers $(N=400)$ into account, values of $d=$ $2.53 \mathrm{~nm}, \gamma=0.25, \sigma=0.29 \mathrm{~nm}$ and $w=0.42 \mathrm{~nm}$ were obtained. These calculations are shown in figure 5.2 as "constant d". As can be seen, these simulations do not agree well with the measurements. In particular, the peak reflectivity at lower $\Gamma$-ratio's is well below the theoretical value.

Possible factors that decrease the peak reflectivity of a ML mirror and, hence, that of a single-order operating LMG can be: (a) interfacial roughness, (b) interlayer formation and interdiffusion between neighboring materials and (c) random or deterministic variation of layer thickness both in the depth and along the ML surface. Although we could not exclude that the etching of the ML stack may result in increased interfacial roughness or interlayer formation, neither photo-electron spectroscopy data nor high-resolution transmission electron microscopy images indicated any difference between the ML stacks before and after etching. Increased roughness and interlayer formation also only reduce peak reflectivity without increasing the bandwidth. In contrary, simulations showed that bi-layer period variations can reduce the peak reflectivity and simultaneously result in increased reflectivity bandwidths.

\subsection{Bi-layer period accuracy}

As an independent check for the degree of bi-layer periodicity, we recorded reflectivity spectra from the Total External Reflection region up to the $4^{\text {th }}$ Bragg order of the unpatterned ML mirror at the hard x-ray energy of $8 \mathrm{keV}$ (HXR). In figure 5.3, the normalized $2^{\text {nd }}$ Bragg order is shown and compared to calculations revealing a slight $(0.5 \%)$ linear increase (chirp) of the bi-layer period $d$ in the depth of the ML stack. Calculations of the SXR spectral bandwidths and peak reflectivities including the measured chirp are shown in figure 5.2 as "chirped d" and can be seen to agree with the measured data to within a few relative percent. The discrepancy in peak reflectivity between the "chirped d" simulations and the reference measurement $(\Gamma=1)$ is believed to be caused by sidewall effects that only occur in the LMGs. The reference was actually on the same sample as the LMGs and, as it was exposed to identical fabrication steps as the LMGs, fabrication can largely be excluded as cause for this difference.

Although the bi-layer chirp in the ML stack was not limiting for use as a conventional ML mirror, LMGs require increased accuracies as the SXR penetration depth is increased and the reflected waves thus need to constructively interfere within a narrower bandwidth. To ensure bi-layer period variations $\Delta d / d$ do not affect LMG performance, they should not exceed $\Delta E / E \approx 1 / N_{\text {eff }}$ (see ref. [18]) and refs. therein), leading to the bi-layer period accuracy condition:

$$
\Delta d_{L M G} \leq \Delta d_{M M} \Gamma
$$

where $\Delta d_{L M G}$ and $\Delta d_{M M}$ are the tolerable variations in bi-layer period for 


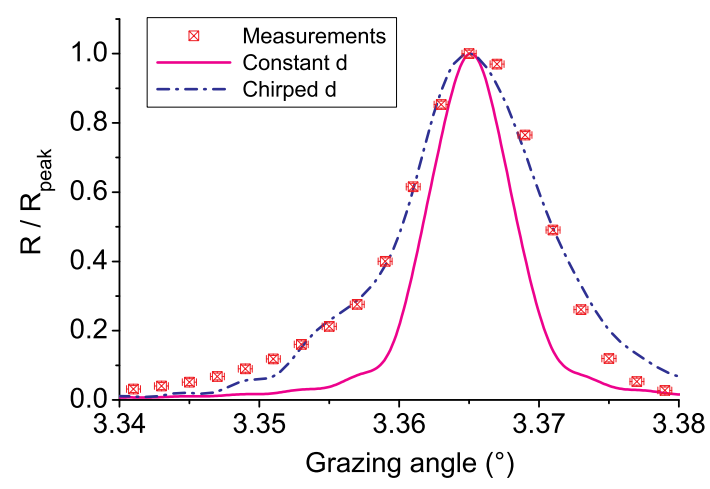

Figure 5.3: Normalized $2^{\text {nd }}$ Bragg order of the unpatterned ML measured at the hard x-ray (HXR) energy of $8 \mathrm{keV}$. Comparison with simulations revealed a slight $(0.5 \%)$ linear increase (chirp) in the bi-layer period $d$ in the depth of the ML.

the LMG and conventional ML mirror, respectively.

\subsection{Energy dependence of LMG performance}

The agreement between the "chirped d" simulations and the measurements proved that our modeling adequately describes the main physical effects underlying the measured reflectivity spectra. This is of relevance for systematic improvements in a next experimental step towards further increased resolution and reflection efficiencies or for extensions to other energies. For instance, the accurate modeling allows us to investigate the usability of LMGs in practical applications by examining the dependence of the spectral resolution $E / \Delta E$ and peak reflectivity $R_{\text {peak }}$ on incident energy.

Such a study was performed for our W/Si LMGs as can be seen in figure 5.4. Here, measurements are shown of 3 LMGs with $\Gamma$-ratios close to 0.28 , which are compared to simulations performed using the "chirped d" values. It can be seen in figure 5.4 that the simulations and measurements are in good agreement, which again demonstrates the accurate modeling by our CWA.

The spectral resolution of these LMGs in this energy range can be seen to be between 250-300. For lower $\Gamma$-ratios, spectral resolutions of up to 330 were achieved. In comparison, conventional W/Si ML mirrors were previously reported to achieve values of $\sim 70$ [13]. However, the usability of LMGs also depends on the peak reflectivity, which has a clear dependence on incident energy. The peak reflectivity can be seen to increase towards higher incident energies with a slight reduction above $1500 \mathrm{eV}$. The changes in peak reflectivity are caused by the energy dependence of the complex refractive index of both materials. Specifically, the absorption reduces when the incident en- 


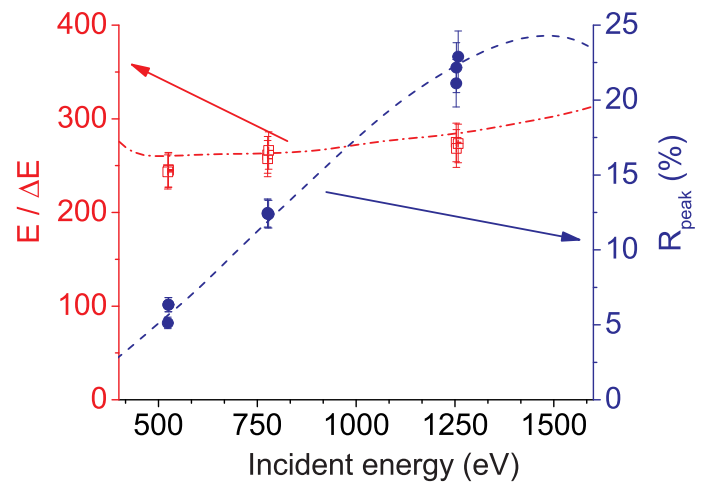

Figure 5.4: Spectral resolution $E / \Delta E$ and peak reflectivity $R_{\text {peak }}$ as function of incident energy for single-order W/Si LMGs with a $\Gamma$ ratio of 0.28 . The simulations were performed assuming the same "chirped d" values as for figure 5.2.

ergy increases from 500 to $1500 \mathrm{eV}$ resulting in higher peak reflectivities. The slight reduction above $1500 \mathrm{eV}$ is due to small variations in the real part of the complex refractive index of tungsten [23]. The usability of LMGs can then be determined by comparing the spectral resolution and peak reflectivity, which can be accurately simulated with our CWA, with the requirements for a specific application.

\subsection{Higher diffraction orders}

So far, LMGs have only been used in specular reflectance. However, the accuracy of our modeling also allows us to investigate the diffraction of LMGs. To extend our knowledge on the operating principles of LMGs, we have carried out higher diffraction order measurements in single-order operation and compared these measurements with our CWA simulations.

To understand higher order excitation, we again focused on single-order LMG operation in which each order is independently excited. To excite higher orders, we must fulfill the quasi-Bragg operation condition $\lambda \approx d\left(\sin \left(\Theta_{0}\right)+\right.$ $\sin \left(\Phi_{m}\right)$ ), where the diffraction angle $\Phi_{m}$, i.e. the grazing angle of the outgoing diffraction order $m$, is determined by the grating equation $m \lambda=D\left(\cos \left(\Theta_{0}\right)-\right.$ $\left.\cos \left(\Phi_{m}\right)\right)$. Higher orders can therefore simply be excited by varying the wavelength and detector angle according to the quasi-Bragg condition.

Such excitation of higher orders in single-order LMG operation is demonstrated in figure $5.5 \mathrm{a}$, where both the $0^{\text {th }}$ and $-1^{\text {st }}$ order of an LMG are shown. Higher orders were measured by first aligning the LMG for specular reflection and then moving the detector to the angle of the preferred outgoing diffraction order. As can be seen, there is no significant excitation of the diffracted or- 
der in the region that overlaps with the neighboring order which demonstrates single-order excitation of higher orders.

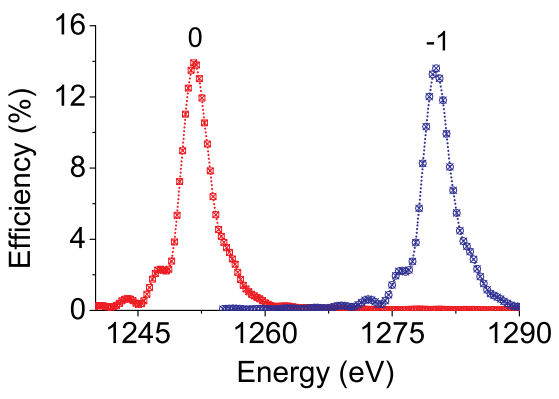

(a)

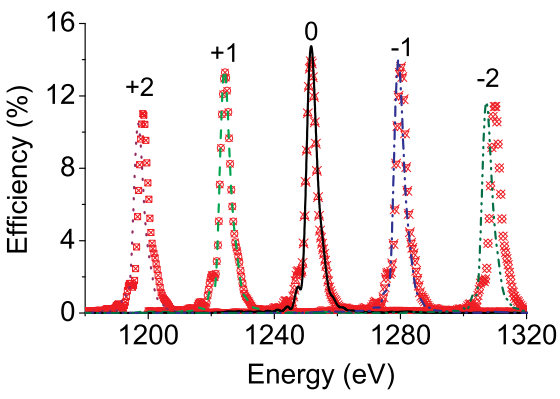

(b)

Figure 5.5: Higher order measurements of an LMG with a grating period of $600 \mathrm{~nm}$ and $\Gamma=0.15$ at the incident energy of $1255 \mathrm{eV}$. (a) The various orders were measured by varying the detector angle and showed no excitation in the overlapping regions. (b) Comparison of measured higher diffraction orders with our CWA simulations using the same ML parameters as the "chirped d" simulations of figure 5.2.

For this LMG, we measured up to the $\pm 2^{\text {nd }}$ diffraction order as can be seen in figure $5.5 \mathrm{~b}$ and compared these measurements with simulations using our CWA. For these simulations, we assumed the same LMG parameters as used for the "chirped d" simulations of figures 5.2 and 5.3. As can be seen in figure $5.5 \mathrm{~b}$, the agreement is quite good, except for a slight underestimation of the peak efficiency of the negative orders and misalignment of the $\pm 2^{\text {nd }}$ order peak positions. Both discrepancies can be explained by a slight difference between the actual and simulated grating period and/or lamel width.

We also measured the FWHM and peak efficiency of the $-1^{\text {st }}$ and $-2^{\text {nd }}$ order as function of $\Gamma$ as can be seen in figure 5.6. This figure shows that the higher orders are suppressed at $\Gamma$-ratios of $1 / m$, where $m$ is the diffraction order. This can also be derived from our analytical CWA from the fact that $B_{ \pm}=0$ (in eq. (3.25)) when $\Gamma=1 / m$. Comparable to gratings for visible light, the suppression of these orders is caused by the convolution of the diffraction patterns from a single trench with that of the entire grating [20,93].

From the figure, it can easily be seen that higher order use of LMGs is of limited practical advantage with respect to bandwidth and peak reflectivity. The bandwidth is only slightly lower at higher $\Gamma$-ratios and approaches the $0^{\text {th }}$ diffraction order bandwidth towards lower $\Gamma$-ratios. However, the loss in peak reflectivity is significant, which is most likely not acceptable for most applications considering the limited improvement in bandwidth. 


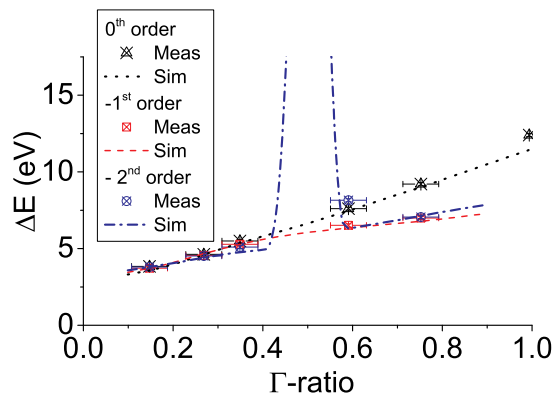

(a)

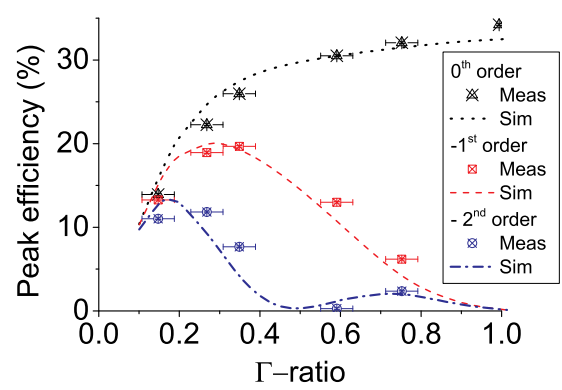

(b)

Figure 5.6: (a) FWHM and (b) peak efficiency as function of $\Gamma$ ratio for the $0^{\text {th }},-1^{\text {st }}$ and $-2^{\text {nd }}$ orders and a grating period $D$ of $600 \mathrm{~nm}$. At the $\Gamma$-ratio of 0.5 , the $\pm 2^{\text {nd }}$ diffraction orders are suppressed, resulting in a sharp decrease of peak efficiency and an ill-defined bandwidth.

\subsection{Conclusions}

We have demonstrated single-order operation of Lamellar Multilayer Gratings (LMG) in the soft x-ray (SXR) spectral range. This was evident from the absence of secondary structures adjacent to the main Bragg peak and an increase in the peak reflectivity with respect to a multi-order LMG with the same lamel-width-to-grating-period ratio $\Gamma$ and, hence, same number of effective layer $N_{\text {eff }}$ contributing to the reflection. The lack of secondary structures and increase in peak reflectivity demonstrate that single-order LMGs do not suffer from significant losses into higher diffraction orders.

The observed spectral bandwidths and peak reflectivity values were in good agreement with our theoretical model (Chapter 3 and refs. [5, 6]). The spectral resolution was improved in single-order operation by a factor of 3.8 with regard to the unpatterned ML mirrors. This improvement with limited peak reflectivity loss points to a significant potential of LMGs in single-order operation for many practical applications, such as SXR spectroscopy. Excitation of higher diffraction orders in single-order operation was easily achieved by simply varying the detector angle with respect to the specular reflection angle when varying the incident energy for a fixed incident angle. Measured bandwidths and peak efficiencies of higher diffraction orders were in good agreement with our CWA. However, the practical benefits of higher order excitation appear to be limited as, compared to the $0^{\text {th }}$ order, the bandwidth is only slightly reduced and the peak efficiency drops significantly. 


\section{6 Performance of finite stack Lamellar Multilayer Gratings}

\subsection{Introduction}

The spectral resolution of multilayer (ML) mirrors for the soft x-ray (SXR) spectral range can be improved by fabricating a grating into such a mirror. The resulting structure is referred to as a Lamellar Multilayer Grating (LMG). Design of LMGs has previously always assumed the idealized case of a semiinfinite ML stack, where the ML and grating parameters were considered independently. The ML would be designed first following Refs. [4, 67] and the effect of the grating would then be considered subsequently. The effect of the grating on LMG optical performance, in terms of bandwidth and peak reflectivity, can be easily predicted for LMGs operating in single-order, i.e. when the incident wave only excites a single diffraction order. The bandwidth then namely simply scales with the lamel-width-to-grating-period ratio $\Gamma$ and the peak reflectivity can be as high as that of a conventional ML mirror. However, this requires an increase in both the number of bi-layers and the accuracy of the deposited bi-layer period by $1 / \Gamma$ as compared to conventional ML mirrors to fulfill the semi-infinite ML approximation [5-7, 74].

The semi-infinite ML approximation generally requires ML stacks of several $\mu \mathrm{m}$ height that consist of hundreds or even thousands of bi-layers that need to be deposited with sub-pm accuracy [5, 7]. Furthermore, gratings then need to be fabricated into these ML stacks with lamel widths in the order of $100 \mathrm{~nm}$ and sub- $\mu \mathrm{m}$ grating periods [74]. Such structures can usually not be deposited or fabricated with sufficient accuracy using current technologies. As a result, most LMGs are fabricated in ML stacks with insufficient bi-layers to fully reflect the incident beam, i.e. part of the incident beam extends into the substrate. This 
situation is referred to as the finite ML stack and results in a limitation of the achievable bandwidth reduction and a loss of peak reflectivity. Furthermore, to optimize LMG optical performance for such finite ML stacks, i.e. to maximize bandwidth reduction or minimize peak reflectivity loss, the LMG design needs to consider both ML and grating parameters simultaneously.

In this chapter, we analyzed the optical performance of single-order LMGs considering various practical limitations. In particular, we investigated the dependence on the number of bi-layers in the ML stack. The optical performance for the semi-infinite ML stack approximation is discussed in section 6.2. Both a general analytical description of the optical performance and simulations for the specific case of W/Si LMGs are presented. Simulations of the same W/Si LMGs were then performed assuming a finite ML stack, as presented in section 6.3, to determine the effect on optical performance of such a stack. Here, we also experimentally demonstrate tuning of LMG optical performance using the ML $\gamma$-ratio.

\subsection{Semi-infinite approximation}

We first investigated the optical performance of LMGs for the idealized case of a semi-infinite ML stack. For this analysis we used a previously derived analytical description of LMG optical performance, as discussed in section 3.2.5 [6], to determine how various parameters affect the performance. The analytical solution to our Coupled Waves Approach (CWA) [6], as presented in section 3.2.4, was subsequently used to simulate W/Si LMGs assuming a semi-infinite ML stack.

\subsubsection{Reflection from single-order LMGs}

The reflection of both ML and LMGs structures is based on the well-known principle of Bragg reflection. In the ML stack, absorber layers are separated by spacer layers such that for a given incident wavelength $\lambda$ and angle $\Theta_{0}$, the bi-layer period $d$ fulfills the Bragg condition [14]:

$$
j \lambda=2 d \sin \Theta_{0}
$$

where $j$ is the ML Bragg order. Furthermore, reflection from such a structure is only achieved when an optical modulation is present between the layers in the ML stack. Suitable materials are selected based on the complex susceptibility $\chi$. The susceptibility can be derived from the complex dielectric constant $\epsilon=1-\chi$, where $\epsilon=n^{2}$ and $n$ is the complex refractive index given by $1-\delta+i \beta$. The optical modulation can be determined from the difference in the real part of the refractive index, whereas the imaginary part indicates absorption. The spacer material is selected such that absorption is minimal, i.e. $\operatorname{Im}\left(\chi_{S}\right)$ is as small as 
possible. The absorber material is then selected by maximizing the ratio [4]:

$$
\Lambda=\frac{\operatorname{Re}\left|\chi_{A}-\chi_{S}\right|}{\operatorname{Im}\left(\chi_{A}\right)}
$$

In this work, we also assumed single-order LMG operation. This is achieved when the incident beam effectively only excites a single-diffraction order, which therefore minimizes diffraction losses and ensures maximum LMG peak reflectivity. Single-order operation is achieved when:

$$
\Gamma D \cdot \Delta \Theta_{\Gamma=1}<<d
$$

where $\Gamma D$ is the lamel width, $D$ is the grating period and $\Delta \Theta_{\Gamma=1}$ is the angular bandwidth of the unpatterned ML mirror. In general, the lamel width should be determined based on the single-order condition and the grating period should then subsequently be chosen based on the required $\Gamma$-ratio.

The theoretical equations describing the optical performance of semi-infinite stack LMGs operating in single-order are discussed next.

\subsubsection{Analytical description of optical performance}

As we were only interested in specular reflection of the first ML Bragg order, the peak reflectivity of a semi-infinite ML stack for s-polarized radiation was written as $[4,6]$ :

$$
R_{0, p e a k}=\frac{1-w}{1+w}
$$

where:

$$
w=\sqrt{\frac{1-y^{2}}{1+f^{2} y^{2}}} ; \quad f=\frac{\operatorname{Re}\left(\chi_{A}-\chi_{S}\right)}{\operatorname{Im}\left(\chi_{A}-\chi_{S}\right)} ; \quad y=\frac{\operatorname{Im}\left(\chi_{A}-\chi_{S}\right)}{\operatorname{Im}(\bar{\chi})} \frac{\sin (\pi \gamma)}{\pi}
$$

The average susceptibility $\bar{\chi}$ is given by $\gamma \cdot \chi_{A}+(1-\gamma) \cdot \chi_{S}$, where $\gamma$ is the absorber-thickness-to-bi-layer-period ratio. From eqs. (6.4)-(6.5), it can be seen that the peak reflectivity actually only depends on the ML parameters $\left(\chi_{A}\right.$, $\chi_{S}$ and $\gamma$ ). As the materials have already been chosen based on the complex refractive index, the peak reflectivity can only be varied using the $\gamma$-ratio. The reflectivity achieves its maximum possible value when the parameter $y$ is maximal, which is achieved when the $\gamma$-ratio of the multilayer structure obeys the equation:

$$
\tan \left(\pi \gamma_{o p t}\right)=\pi\left[\gamma_{o p t}+\frac{\operatorname{Im}\left(\chi_{S}\right)}{\operatorname{Im}\left(\chi_{A}-\chi_{S}\right)}\right]
$$

as is well-known from the theory of conventional SXR multilayer mirrors [20, 67]. The optimal ratio $\gamma_{\text {opt }}$ predicted by this equation is typically in the order of $0.15-0.45$ as $\operatorname{Im}\left(\chi_{A}\right)>\operatorname{Im}\left(\chi_{S}\right)$. As the peak reflectivity reduces to zero for both $\gamma \rightarrow 0$ and $\gamma \rightarrow 1$, the peak reflectivity is generally more strongly affected by a reduction than an increase in $\gamma$-ratio with respect to $\gamma_{o p t}$. 
The bandwidth of an LMG can be approximately derived from the penetration depth $L_{M S}$, which is given by:

$$
L_{M S}=N_{e f f} \cdot d \sim \frac{\lambda \sin \Theta_{0}}{\pi \Gamma \operatorname{Im}(\bar{\chi}) \sqrt{\left(1-y^{2}\right)\left(1+f^{2} y^{2}\right)}},
$$

where $N_{e f f}$ is the required number of bi-layers effectively contributing to the reflection. By substituting $d=\lambda / 2 \sin \Theta$ into eq. (6.7) and using the resolving power $E / \Delta E=\tan \Theta / \Theta \approx N_{\text {eff }} \pi / 2$ as given in refs. [4, 18], the bandwidth was derived to be approximately given by:

$$
\Delta \Theta_{L M G} \approx \frac{2 \Gamma \operatorname{Im} \bar{\chi}}{\sin \left(2 \Theta_{0}\right)} \sqrt{\left(1-y^{2}\right)\left(1+f^{2} y^{2}\right)}
$$

Here, the terms $f$ and $y$ are the same as in eq. (6.5). The bandwidth was thus determined to depend on both the ML and the grating parameters.

It can be determined from eq. (6.8) that the bandwidth scales linearly with the grating $\Gamma$-ratio and, assuming only $\Gamma$ is changed, the bandwidth can then be predicted from the ML bandwidth using $\Delta \Theta_{L M G}=\Gamma \Delta \Theta_{\Gamma=1}$. The dependence of the bandwidth on the ML parameters via $\operatorname{Im}(\bar{\chi}) \cdot \sqrt{\left(1-y^{2}\right)\left(1+f^{2} y^{2}\right)}$ is more complicated. However, assuming typical values of $f$ in the order of $2-4$ for the SXR spectral range and $\gamma \leq 1 / 2$, we determined a reduction of $\gamma$ also results in a reduction of bandwidth. This can be understood as a reduction in $\gamma$-ratio results in a lower average absorption per bi-layer. The SXR penetration depth is then increased which reduces the bandwidth.

As the bandwidth of LMGs reduces due to the increased SXR penetration depth, the reflected waves need to constructively interfere within a narrower bandwidth. To ensure bi-layer period variations $\Delta d / d$ are not limiting, they should not exceed $\Delta E / E \approx 1 / N_{\text {eff }}$ (see ref. [18] and refs. therein), leading to the bi-layer period accuracy condition [7]:

$$
\Delta d_{L M G}=\Delta d_{M M} \cdot \Gamma
$$

where $\Delta d_{L M G}$ and $\Delta d_{M M}$ are the tolerable variations in bi-layer period for the LMG and conventional ML mirror, respectively.

Using the penetration depth given by eq. (6.7), a criteria for the validity of the semi-infinite approximation was also determined. The semi-infinite approximation basically assumes that the incident beam does not extend into the substrate. This means the actual number of bi-layers $N$ in the ML stack should be equal or larger than the required number of bi-layers $N_{\text {eff }}$ given by eq. (6.7). The semi-infinite approximation is thus only valid when:

$$
N \geq N_{e f f}
$$

For LMGs with semi-infinite ML stacks, the maximum peak reflectivity is obtained when $\gamma=\gamma_{\text {opt }}$ and the $\Gamma$-ratio can be used to set the desired bandwidth. We next performed simulations of W/Si LMGs with a semi-infinite ML stack to confirm this theoretical analysis. These simulations were subsequently also used for comparison with the finite ML case. 


\subsubsection{Simulation of semi-infinite LMGs}

For the simulations in this section, we considered LMGs based on an initial W/Si ML structure corresponding to those used in our experiments. The densities were $\rho_{W}=19 \mathrm{~g} / \mathrm{cm}^{3}$ and $\rho_{S i}=2.35 \mathrm{~g} / \mathrm{cm}^{3}$ with a bi-layer period $d=$ $2.53 \mathrm{~nm}$ and no interfacial roughness. The grating period was chosen to be $D=300 \mathrm{~nm}$ to ensure single-order operation for all $\Gamma$-ratios at the incident energy of $1255 \mathrm{eV}$.

Figure 6.1a shows a contour plot of the simulated bandwidth and peak reflectivity as function of the ML $\gamma$ and grating $\Gamma$-ratios. The red dashed lines connect positions of constant bandwidth, whereas the blue solid lines indicate positions of constant peak reflectivity. As the lines of constant peak reflectivity are not affected by the grating $\Gamma$-ratio, it can be concluded that the peak reflectivity only depends on the ML $\gamma$-ratio. It can also be seen that the bandwidth is affected by both the ML $\gamma$-ratio and the grating $\Gamma$-ratio. However, the grating $\Gamma$-ratio can actually be used to achieve any desired bandwidth for any $\gamma$-ratio. One can thus simply determine the ML $\gamma$-ratio based on the required peak reflectivity, which is maximum when fulfilling eq. (6.6), and subsequently use the $\Gamma$-ratio to reduce the bandwidth to any desired value. These results are in good agreement with the previous section.

\subsection{Finite ML stacks}

The semi-infinite approximation implicates the use of ML stacks that can easily consist of hundreds or even thousands of layers, resulting in total stack heights in the tens of microns for combinations of low $\gamma$ and low $\Gamma$-ratios. Furthermore, these stacks need to be fabricated with increased accuracy down to sub-pm of the bi-layer period. Finally, gratings need to be fabricated in these ML stacks with $100 \mathrm{~nm}$ lamel widths and sub- $\mu \mathrm{m}$ grating periods. Such LMGs can often not be fabricated with sufficient accuracy using current ML deposition and grating fabrication technologies. Most LMGs therefore do not fulfill the semiinfinite approximation and use ML stacks with insufficient bi-layers to fully reflect the incident beam, referred to as the finite ML stack case.

We first theoretically investigated the effect of a finite ML stack by comparing finite ML simulations with those of a semi-infinite case. Next, we experimentally demonstrate the tailoring of LMG optical performance using the ML $\gamma$-ratio by comparing measured optical performance for various LMGs fabricated in $2 \mathrm{ML}$ stacks with different $\gamma$-ratios.

\subsubsection{Simulation of LMGs with finite ML stacks}

To determine the effect of finite ML stacks on LMG optical performance, we compared the simulations of semi-infinite LMGs shown in figure 6.1a with simulations assuming the same ML, but with a finite ML stack, as can be seen in 


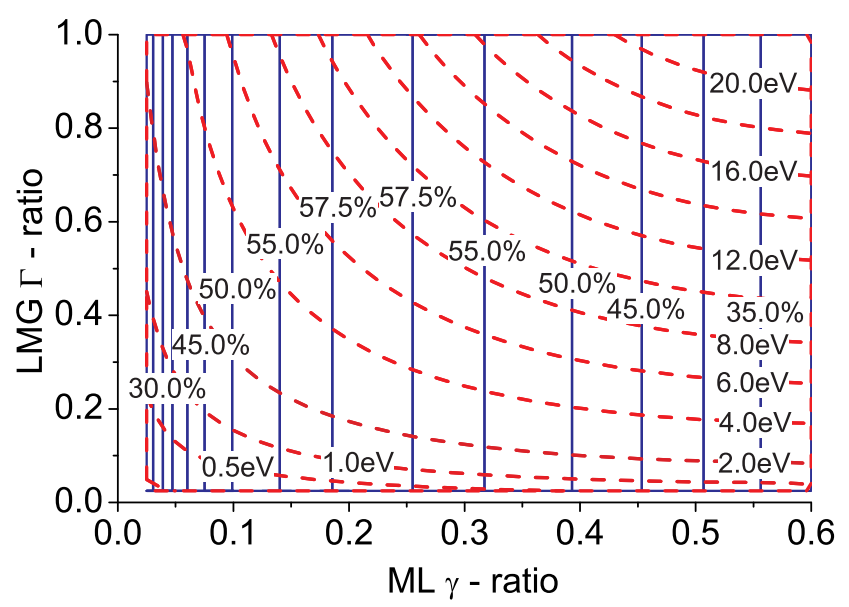

(a) For a semi-infinite ML stack, the ML $\gamma$-ratio can be used to determine the peak reflectivity and the bandwidth can then be reduced using the $\Gamma$-ratio of the grating.

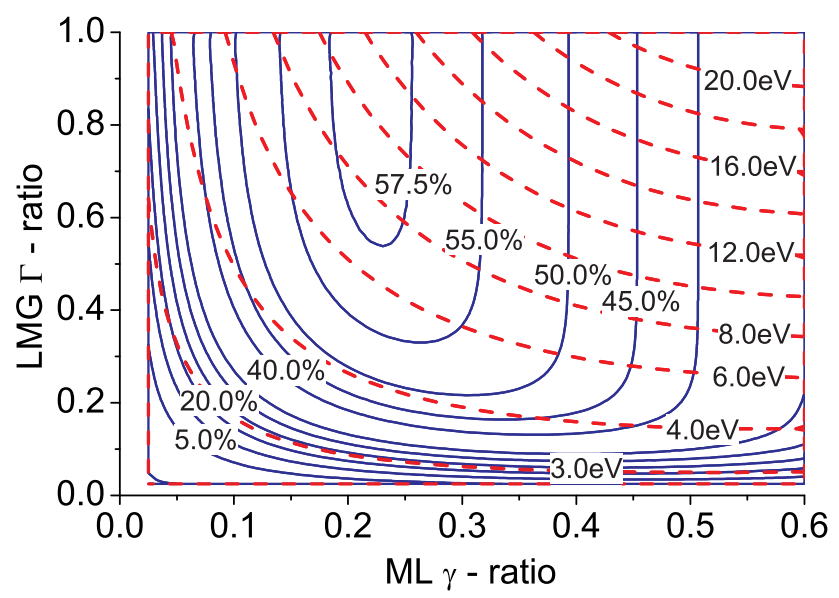

(b) For a finite ML stack, bandwidth and peak reflectivity cannot be considered separately and an optimal combination of bandwidth and peak reflectivity needs to be chosen for each specific application.

Figure 6.1: LMG bandwidth (red dotted) and peak reflectivity (blue solid) simulations for s-polarized radiation as function of the ML $\gamma$ and grating $\Gamma$-ratio for (a) a semi-infinite ML stack and (b) a finite ML stack with $N=400$ bi-layers. The ML parameters used were $\rho_{W}=19 \mathrm{~g} / \mathrm{cm}^{3}, \rho_{S i}=2.35 \mathrm{~g} / \mathrm{cm}^{3}$, bi-layer period $d=2.53 \mathrm{~nm}$ and no interfacial roughness. The grating period was $D=300 \mathrm{~nm}$ and chosen to ensure single-order operation for all $\Gamma$-ratios at the incident energy of $1255 \mathrm{eV}$. 
figure $6.1 \mathrm{~b}$. The figure shows a contour plot of the simulated bandwidth and peak reflectivity for a fixed $N$ of 400 bi-layers, chosen as a typical experimental case.

Comparing the finite with the semi-infinite case, some significant differences can be seen. First of all, the peak reflectivity depends on both the ML $\gamma$-ratio and the grating $\Gamma$-ratio for the finite $\mathrm{ML}$ case and decreases for lower $\Gamma$-ratios. Specifically, the peak reflectivity in the semi-infinite case was maximum for $\gamma=0.22$ for all $\Gamma$-ratios, whereas this peak reflectivity is only achieved for $\Gamma>0.5$ in the finite ML case. Furthermore, the minimal achievable bandwidth in the finite $\mathrm{ML}$ case is limited to just below $3 \mathrm{eV}$ for a combination of low $\gamma$ and $\Gamma$-ratios, whereas the same structure in the semi-infinite ML stack easily reached bandwidths below $0.5 \mathrm{eV}$. Both effects are caused by the incident beam extending into the substrate, which limits the number of bi-layers contributing to the reflection.

\subsubsection{Tailoring of performance with the $\gamma$-ratio}

Examining figure $6.1 \mathrm{~b}$ in some more detail, it can be seen that for low $\Gamma$-ratios the ML $\gamma$-ratio can be used to tailor LMG optical performance. Lower ML $\gamma$-ratios increase SXR penetration depth and therefore result in a further reduction of bandwidth at the expense of a lower peak reflectivity. On the other hand, higher $\gamma$-ratios result in slightly increased bandwidths, but significantly less peak reflectivity loss as each individual bi-layer has a slightly higher reflectance. Comparing $\gamma$-ratios of 0.1 and 0.4 , it can be seen that the higher $\gamma$-ratio results in an increase in bandwidth of $15 \%$ and a factor 3 increase in peak reflectivity with respect to the lower $\gamma$-ratio.

We experimentally investigated the effect of the $\gamma$-ratio on LMG optical performance using LMGs fabricated in two W/Si MLs with different $\gamma$ ratios [72, 94]. Using Grazing Incidence X-ray Reflectometry (GIXR) at the $\mathrm{Cu}-K \alpha$ energy of $8 \mathrm{keV}$, we first analyzed the ML structures by measuring the reflectance as function of angle in the range from the Total External reflection region up to the $4^{t h}$ Bragg order. These measurements showed the first ML (ML1) to have a $\gamma$-ratio of 0.27 , whereas the other ML (ML2) had a significantly lower $\gamma$-ratio of 0.18 .

The measured LMG optical performance at the incident energy of $1255 \mathrm{eV}$ is depicted in figure 6.2 for various LMGs fabricated in both MLs. The measured peak reflectivities are lower than the simulated values shown in figure 6.1 as the interfacial roughness was not taken into account in those simulations. As can be seen, the reference ML measurements $(\Gamma=1)$ have comparable peak reflectivities, but very different bandwidths due to the difference in $\gamma$-ratio of both ML stacks. It is important to note that the ML references $(\Gamma=1)$ still fulfill the semi-infinite approximation.

When the $\Gamma$-ratio was reduced, the peak reflectivity initially remained reasonably constant and then began to decrease below a certain $\Gamma$-ratio. Below 
that $\Gamma$-ratio the incident beam began to extend into the substrate and, hence, this indicated the transition from the semi-infinite to the finite ML case. The $\Gamma$-ratio at which this occurred was roughly 0.4 for ML1 and 0.6 for ML2. The bandwidth of both MLs also reduced towards lower $\Gamma$-ratios. In the semi-infinite region the bandwidth scaled linearly with $\Gamma$. Once the finite ML region was reached, the bandwidth began to asymptotically approach a minimal value as the minimal achievable bandwidth was then limited by the number of bi-layers in the ML.

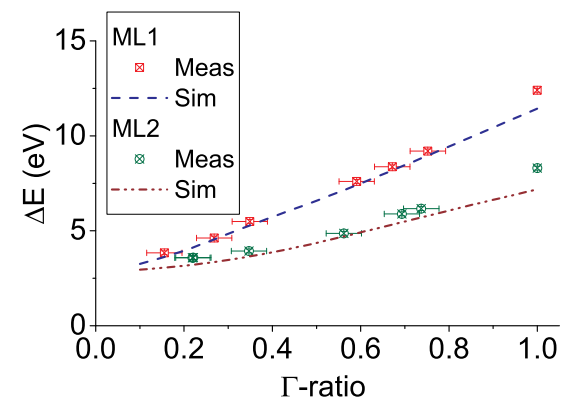

(a)

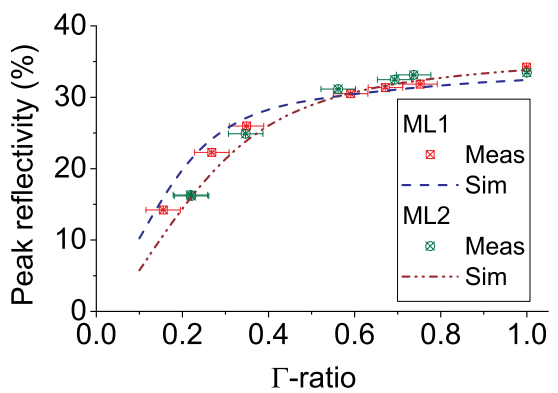

(b)

Figure 6.2: Comparison of (a) bandwidth and (b) peak reflectivity for two MLs with different $\gamma$-ratios.

However, the peak reflectivity of LMGs with low $\Gamma$-ratios differs for both MLs. ML2 had a lower $\gamma$-ratio, which resulted in narrower bandwidths and lower peak reflectivities. On the other hand, ML1 had a larger $\gamma$-ratio and therefore resulted in a slightly higher bandwidth by a few tenths of eV. However, the peak reflectivity was increased by almost a factor 2 . In fact, the peak reflectivity of ML1 should actually have been even higher, but was limited due to a linear increase (chirp) of the bi-layer period in the depth of the ML as explained in more detail in ref. [7]. ML2 had a significantly lower chirp, which did not affect the optical performance of the LMGs fabricated in that ML. LMG optical performance can thus be tailored to maximize bandwidth reduction or minimize peak reflectivity loss using the ML $\gamma$-ratio.

\subsection{Conclusions}

We have analyzed the dependence of Lamellar Multilayer Grating (LMG) optical performance, in terms of bandwidth and peak reflectivity, on various practical limitations. Specifically, we have investigated how the number of bi-layers in the multilayer (ML) stack affects LMG performance. We first studied the idealized case of a semi-infinite ML stack, which is an often used approximation for ML and LMG design. For the semi-infinite case, LMGs can be designed by 
first optimizing the absorber-thickness-to-bi-layer period ratio $\gamma$ of the ML to achieve maximum peak reflectivity. The bandwidth can then be subsequently reduced to any desired value using the lamel-width-to-grating-period ratio $\Gamma$. The lamel width of the grating is then determined from the single-order condition and the grating period is selected based on the required $\Gamma$-ratio.

However, the semi-infinite ML approximation generally requires ML stacks of hundreds to thousands of bi-layers, resulting in total stack heights of several microns. These stacks also need to be deposited with sub-pm accuracy. Gratings then need to be fabricated in these ML stacks with lamel widths in the order of $100 \mathrm{~nm}$ and sub- $\mu \mathrm{m}$ grating periods. Such structures can generally not be deposited or etched with adequate precision using current technologies. LMGs are therefore often fabricated in ML stacks with insufficient bi-layers to fulfill the semi-infinite approximation, referred to as the finite ML case. A criteria for the transition from the semi-infinite to finite ML case was derived based on the SXR penetration depth.

To determine the effect of a finite ML stack, simulations of LMG optical performance comparing the semi-infinite and finite ML case were performed. For the finite ML stack, the minimal achievable bandwidth and maximum peak reflectivity are limited by the number of bi-layers in the ML stack. Optical performance can then be further tailored using the ML $\gamma$-ratio. A lower $\gamma$-ratio will result in slightly narrower bandwidths at the expense of peak reflectivity. If slightly higher bandwidths can be accepted, higher $\gamma$-ratios should be used to minimize peak reflectivity loss. This was also experimentally demonstrated for LMGs fabricated in two W/Si MLs with different $\gamma$-ratios of 0.18 and 0.27. For LMGs with low $\Gamma$-ratios, the higher ML $\gamma$ resulted in a few tenths of eV larger bandwidth, but almost doubled the peak reflectivity as compared to the lower ML $\gamma$. 



\section{7 \\ Lifetime of patterned $\mathrm{W} / \mathrm{Si}$ multilayer mirrors}

\subsection{Introduction}

The fabrication of diffractive elements in multilayer (ML) mirrors can provide Bragg-Fresnel optics for the EUV and soft x-ray (SXR) spectral ranges with unique properties. An important example are Lamellar Multilayer Gratings (LMGs) which provide improved spectral resolution with minimal loss of peak reflectivity [5-7] for soft x-ray (SXR) spectroscopy applications.

The practical usability of such optics depends on their lifetime, which can be reduced by several degradation processes [95-99]. In particular, we distinguish degradation due to surface phenomena and internal changes to the ML structure. Surface phenomena include sidewall contamination due to the fabrication process or the cracking of hydrocarbons, for instance from photoresist or cables in the vacuum system holding the ML mirror [96, 97, 100, 101]. The contamination results in additional absorption and hence reduces peak reflectivity of LMGs. Internal ML structure changes can be caused by intermixing due to thermal annealing or diffusion of elements such as oxygen into the ML structure. This can result in a change in the bi-layer period or reduce the optical contrast between the absorber and spacer layer in the ML stack [102-106]. As a consequence of these structural ML changes, the optical performance of LMGs in terms of bandwidth, peak reflectivity and/or peak position, can be affected.

Compared to conventional ML mirrors, the fabrication of optical elements such as LMGs increases their vulnerability to degradation processes. The fabrication process described in chapter 4 [74] includes several steps in which the 
ML structure is exposed to conditions that may enhance degradation of the ML structure. These include steps in which increased temperatures are used, and where fluorine and oxygen radicals can chemically react with the ML materials. Furthermore, carbon-fluorine components are deposited on the sidewalls of the LMG structure in the course of the fabrication process to achieve a directional etch. The fabrication of LMGs also increases the surface area between the ML and vacuum by easily an order of magnitude as compared to conventional ML mirrors and directly exposes the ML structure to the various degradation processes.

In this chapter, we present a fabrication and storage lifetime study of Lamellar Multilayer Gratings (LMGs) fabricated in W/Si ML mirrors. In section 7.2 we first investigate various potential degradation processes, including sidewall contamination, thermal annealing and oxygen diffusion. SXR reflectivity was determined to remain stable to within measurement accuracy over an extended period of time as discussed in section 7.2. Radiation induced processes, such as the cracking of hydrocarbons and intermixing of the ML stack [95-97, 100, 101], were not investigated in this work. A preliminary study into the use of capping layers to protect the patterned MLs from degradation and carbon removal methods is presented in section 7.3.

Although in this study we focused on the lifetime and degradation of LMGs, various other types of Bragg-Fresnel optics can be made using the same fabrication process and be stored under the same conditions. This study can therefore also be used to predict degradation of the broader class of Bragg-Fresnel optics based on patterned W/Si ML mirrors.

\subsection{Potential degradation processes}

In this section, we examine the effect of various potential degradation processes. We first examined sidewall contamination, which is a process limited to the surface of the LMG. Next, processes affecting the ML internal structure, such as thermal annealing and oxygen diffusion, were investigated. Finally, the stability of SXR reflectivity was investigated over a storage period extending up to 18 months.

\subsubsection{Sidewall contamination}

To determine the effect of a sidewall contamination layer, we first measured its composition using x-ray photoelectron spectroscopy (XPS) and then theoretically investigated the optical effect of using an extension to our Coupled Waves Approach (CWA) [5, 6]. 


\section{Sidewall contamination layer composition}

To measure the sidewall composition, an LMG with a $1 \mu \mathrm{m}$ period and a lamel width of $200 \mathrm{~nm}$ was examined with XPS under a $45^{\circ}$ incidence angle, giving an optimal geometry to measure the spectrum from the sidewalls. After determining the present elements, the atomic concentration $C_{x}$ of each element was determined with:

$$
C_{x}=\frac{\frac{I_{x}}{R S F_{x}}}{\sum_{i}^{n} \frac{I_{i}}{R S F_{i}}}
$$

where $I_{i}$ is the peak area of the photon peak and $R S F_{i}$ is the Relative Sensitivity Factor of each element.

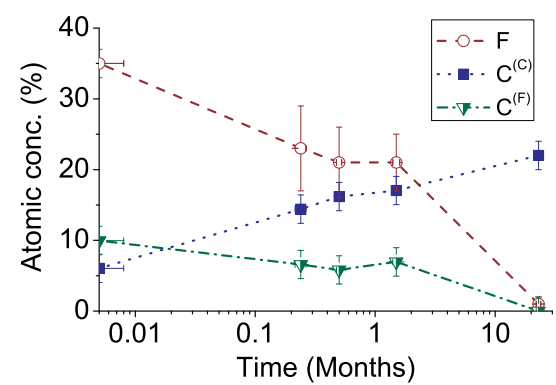

(a)

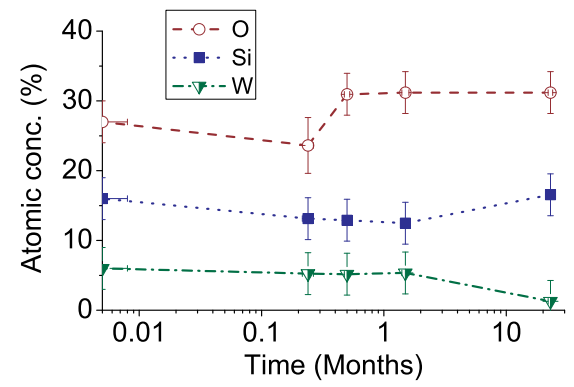

(b)

Figure 7.1: Atomic concentration of elements present in the sidewalls for the first 23 months of storage. (a) The concentration of fluorine and bonding of carbon, divided into carbon-carbon bonds $\mathrm{C}^{(C)}$ and carbon-fluorine bonds $\mathrm{C}^{(F)}$, clearly changed. (b) The concentration of silicon, tungsten and oxygen were corrected for the desorped fluorine fraction and remained stable within measurement accuracy.

After measuring which elements were present in the sidewalls, we investigated their atomic concentration as function of time, as shown in figure 7.1, for the first 23 months of storage in a 1 atm air environment, to determine the stability of the sidewall composition. The first four points were measured on a single sample. To obtain the point at 23 months storage, an XPS measurement was performed on a sample that had previously been fabricated using an identical process and stored under similar conditions. It can be seen in figure $7.1 \mathrm{a}$ that the most significant changes are a gradual desorption of fluorine and changes in the carbon bonds. Since fluorine dissociated and desorped from the probed area to a significant degree, the measurements of tungsten, silicon and oxygen concentration were corrected for the desorped fluorine fraction, i.e. the atomic concentration of those three elements were normalized to a total value of $49 \%$ corresponding to their total concentration in the first measurement after fabrication. The atomic concentrations of these elements are shown 
in figure $7.1 \mathrm{~b}$ and can be seen to be relatively stable. The fluctuations in these measurements were attributed to non-constant storage conditions, e.g. changes in temperature and humidity, and the use of 2 samples to obtain the data.

The desorption of fluorine was most clearly seen in the carbon spectra as shown in figure 7.2. In the figure, the measured spectra directly after fabrication (figure 7.2a) and after one month storage (figure 7.2b) are depicted. A clear change in the peaks required to describe the spectrum can be seen, which indicates changes to the chemical bonds have occurred. The peak at $285 \mathrm{eV}$ corresponds to aliphatic carbon, i.e. C-C bonds, whereas the peak at $291 \mathrm{eV}$ corresponds to $\mathrm{C}_{-} \mathrm{F}_{2}$ and the peak at $294 \mathrm{eV}$ can indicate either $\mathrm{C}_{-} \mathrm{F}_{2} \mathrm{O}$ or $\mathrm{C}$ $\mathrm{F}_{3}$ bonds. These carbon-fluorine bonds can be easily understood as $\mathrm{C}-\mathrm{F}_{2}$ was used in our fabrication process to achieve directional etching [75], as previously described in section 4.3, and can easily react with oxygen or fluorine to form the other compounds. The peak at $287 \mathrm{eV}$ can correspond to various bonds and could not be uniquely attributed to a specific compound [107, 108].

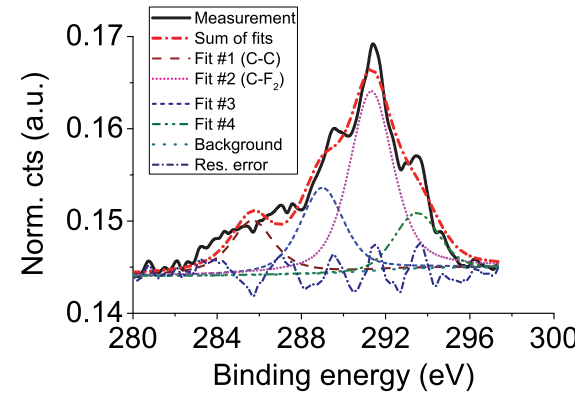

(a)

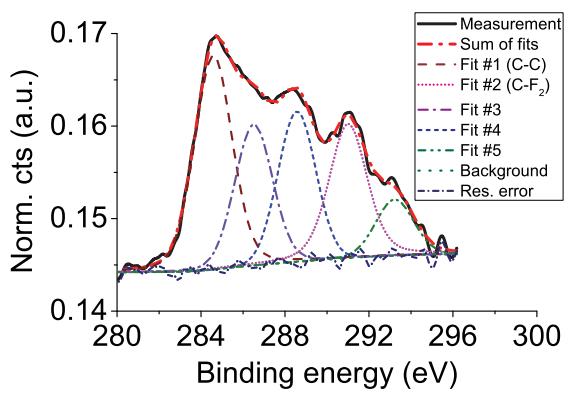

(b)

Figure 7.2: Measured XPS spectra of carbon after (a) 0 and (b) $1 \frac{1}{2}$ months of storage. The spectrum showed a reduction in the carbon-fluorine bonds around $290 \mathrm{eV}$ and increase in carboncarbon bonds at $284 \mathrm{eV}$.

However, based on the atomic concentrations and ratios of elements in the specific bonds we determined not all fluorine was initially bound to carbon. It is known that the fabrication process can also result in silicon oxyfluoride compounds [75]. The peak in the XPS spectrum of silicon corresponding to the oxidized state initially had a FWHM of $3.08 \mathrm{eV}$, which reduced to $2.31 \mathrm{eV}$ during the first week of storage. This narrowing indicated a homogenization of the corresponding compound. We therefore suspect the remaining fluorine to have initially been mainly bound to silicon oxyfluorides from which the fluorine also desorped. We next investigated the optical effect of the sidewall contamination layer on LMG performance. 


\section{Effect of sidewall cover layers on LMG optical performance}

To theoretically investigate the effect of a contamination layer on LMG optical performance, an extension to our Coupled Waves Approach (CWA) [5, 6] was required (see also section 3.2.2). The theory for contamination layers can also be used for deliberately grown capping layers used to protect LMG structures. Here, we therefore refer to all layers as being cover layers when deriving the theory.

To include the effect of cover layers in our CWA, the spatial distribution of the dielectric constant was rewritten to:

$$
\begin{gathered}
\epsilon(x, z<0)=1 ; \quad \epsilon(x, z>H)=1-\chi_{\text {sub }} \\
\epsilon(x, 0 \leq z \leq H)=1-\chi(z) U(x, z)-\chi_{\text {pass }} V(x, z)
\end{gathered}
$$

where $\chi(z)$ is the susceptibility of a multilayer structure varying with the depth, while $\chi_{\text {cov }}$ and $\chi_{\text {sub }}$ are the constant susceptibility of the cover layer and substrate, respectively. The function $U(x, z)=1$ if the point $(x, z)$ is placed inside multilayer structure and $U=0$ otherwise. Similarly, the function $V(x, z)=1$ if the point $(x, z)$ is placed inside the cover layer and otherwise equals 0 .

Assuming the dielectric constant to be periodic function of $x$, we represented the functions $U$ and $V$ as the Fourier series:

$$
\begin{aligned}
& U(x, z)=\sum_{n=-\infty}^{+\infty} U_{n}(z) e^{2 i \pi n x / D} \\
& V(x, z)=\sum_{n=-\infty}^{+\infty} V_{n}(z) e^{2 i \pi n x / D}
\end{aligned}
$$

where

$$
\begin{aligned}
& U_{n}(z)=\frac{\sin (\pi n \Gamma)}{\pi n} \\
& V_{n}(z)=\frac{2}{\pi n} \sin \left(\frac{\pi n h}{D}\right) \cos \left[\pi n\left(\frac{\Gamma}{D}+\frac{h}{D}\right)\right]
\end{aligned}
$$

with $U_{0}=\Gamma$ and $V_{0}=2 h / D$. Please note that the terms $U$ in eq. (4.3) differ to those used in our previous works $[5,6]$ as the point $x=0$ was now chosen to be in the center of the lamel instead of at the left side of the lamel. Shifting the coordinates of the previous works by $x^{\prime}=x-\Gamma D / 2$ yields the same coefficients as found here.

Following the same steps as in section $3.2[5,6]$, the 2D-wave equation $\nabla^{2} E(x, z)+k^{2} \epsilon(x, z) E(x, z)$ was solved, where $\epsilon$ is a periodic function of $x$ and $z$ described by eq. (7.2) and $k$ is the wave vector given by $2 \pi / \lambda$. The general solution for the field $F$ of the incident and reflected waves then has the following form (chapter 1 in ref [66]):

$$
F_{n}^{\prime \prime}(z)+\kappa_{n}^{2} F_{n}(Z)=k^{2} \sum_{m}\left[\chi(z) U_{n-m}(z)+\chi_{\text {pass }} V_{n-m}(z)\right] F_{m}(z)
$$


for $n=0, \pm 1, \pm 2, \ldots$ with boundary conditions:

$$
F_{n}^{\prime}(0)+i \kappa_{n} F_{n}(0)=2 i \kappa_{n} \delta_{n, 0} ; F_{n}^{\prime}(L)-i \kappa_{n}^{(s)} F_{n}(L)=0 .
$$

Here, $\kappa_{n}=\sqrt{k^{2}-q_{n}^{2}}$ and $\kappa_{n}^{(s)}=\sqrt{k^{2} \epsilon_{s u b}-q_{n}^{2}}$ are the z-components of the wave vectors for the $n^{\text {th }}$ diffraction order in vacuum and substrate, respectively, where $q_{n}=q_{0}+2 \pi n / D$ with $q_{0}=k \cos \Theta_{0}$, and $\delta_{n, 0}$ is the Kronecker symbol.

To understand the effect of cover layers, we first recall the optical performance of a single-order LMG without a cover layer $(h=0)[5,6]$. In this case, the effective susceptibility $\chi_{e f f}(z)=\Gamma \chi(z)$ corresponds to a conventional ML mirror with material densities reduced by a factor of $\Gamma$. The maximum achievable peak reflectivity, assuming abrupt interfaces, is then given by [4, 6]:

$$
\begin{gathered}
R_{0, \text { peak }}=\frac{1-w}{1+w} ; \\
w=\sqrt{\frac{1-y^{2}}{1+f^{2} y^{2}}} ; \quad f=\frac{\operatorname{Re}\left(\chi_{A}-\chi_{S}\right)}{\operatorname{Im}\left(\chi_{A}-\chi_{S}\right)} ; \quad y=\frac{\operatorname{Im}\left(\chi_{A}-\chi_{S}\right)}{\operatorname{Im}(\bar{\chi})} \frac{\sin (\pi j \gamma)}{(\pi j)}
\end{gathered}
$$

Scaling the density of both materials by the same factor $\Gamma$ does not change the values of the parameters $f$ and $y$ and, hence, the peak reflectivity of a single-order LMG is the same as that of a conventional ML mirror. At the same time, the penetration depth $L_{M S}$ of the SXR wave into the LMG, which determines the angular or spectral bandwidth, is given by:

$$
L_{M S}=N_{e f f} \cdot d \sim \frac{\lambda \sin \Theta_{0}}{\pi \Gamma \operatorname{Im}(\bar{\chi}) \sqrt{\left(1-y^{2}\right)\left(1+f^{2} y^{2}\right)}}
$$

and is increased by a factor of $1 / \Gamma$. As $N_{\text {eff }}$ is then also increased by $1 / \Gamma$, the number of bi-layers in the LMG must therefore also be increased by the same factor. The bandwidth is only scaled with the factor $1 / \Gamma$ as the parameters $f$ and $y$ remain unchanged. Single-order LMGs therefore allow us to obtain any desired resolution while maintaining high peak reflectivity under the assumption that the ML mirror contains sufficient bi-layers [5].

We then analyzed the effect of a cover layer with thickness $h$. The effective susceptibility of both materials in the ML structure is then increased by the relative amount of cover layer thickness with respect to the lamel, i.e. $\chi_{\text {eff }}(z)=$ $\Gamma \chi(z)+2 h / D \cdot \chi_{\operatorname{cov}}$. The parameter $f$ in eq. (7.7) only depends on the difference between the absorber and spacer layer susceptibility and is hence unaffected by the cover layer. However, $y$ depends on the average absorption, i.e. the imaginary part of the effective susceptibility, and was rewritten to:

$$
y=\frac{\operatorname{Im}\left(\chi_{A}-\chi_{S}\right)}{\operatorname{Im}\left(\chi_{S}+2 h /(\Gamma D) \cdot \chi_{\operatorname{cov}}\right)} \cdot \frac{\sin (\pi j \gamma)}{(\pi j)}
$$

The parameter $y$ is thus reduced due to the cover layer and from eqs. (7.7) and (7.8) it can be seen that this results in a decrease of peak reflectivity and increase in bandwidth [4]. 
The cover layer should generally be as thin as possible to ensure minimal effect on LMG optical performance. From eq. (7.9), we derived that the effect of a cover layer on the peak reflectivity is negligible if the layer thickness is:

$$
h<<\frac{\Gamma D}{2} \cdot \frac{\operatorname{Im}\left(\chi_{S}\right)}{\operatorname{Im}\left(\chi_{\operatorname{cov}}\right)}
$$

A similar condition showing acceptable layer thicknesses to ensure negligible effects on the bandwidth was also derived:

$$
h<<\frac{\Gamma D}{2} \cdot \frac{\gamma \cdot \operatorname{Im}\left(\chi_{A}\right)}{\operatorname{Im}\left(\chi_{\operatorname{cov}}\right)}
$$

To ensure the effect of a cover layer on LMG optical performance is negligible, both conditions should be fulfilled. By examining conditions (7.10) and (7.11), we concluded that the latter is automatically achieved when the former is fulfilled. This is because a highly absorbing material is typically used as absorber, meaning $\operatorname{Im}\left(\chi_{A}\right)>>\operatorname{Im}\left(\chi_{\text {cov }}\right)$. The lowest absorbing material is typically used as spacer, meaning $\operatorname{Im}\left(\chi_{S}\right)<\operatorname{Im}\left(\chi_{\text {cov }}\right)$.

To illustrate the effect of a cover layer on the optical performance of an LMG, we performed simulations of LMGs based on W/Si MLs with parameters corresponding to those used in this work. The densities were taken to be $\rho_{W}=19 \mathrm{~g} / \mathrm{cm}^{3}$ and $\rho_{S i}=2.35 \mathrm{~g} / \mathrm{cm}^{3}, N=400$ bi-layers with period $d=$ $2.53 \mathrm{~nm}$, absorber-thickness-to-bi-layer-period ratio $\gamma=0.28$ and no interfacial roughness. The parameters of the grating were chosen to ensure single-order operation for the entire wavelength range of interest and were a grating period $D=300 \mathrm{~nm}$ with a lamel-width-to-grating-period ratio $\Gamma=0.25$.

Figure 7.3 demonstrates the effect of a silicon oxyfluoride cover layer, which were the three main measured elements present in the sidewall, on LMG performance. As can be seen in figure $7.3 \mathrm{a}$, the effect on the $0^{\text {th }}$ and $\pm 1^{\text {st }}$ orders is indeed very small for the measured cover layer thickness of $5 \mathrm{~nm}$. The peak reflectivity drops by only $1 \mathrm{pp}$ (3 relative \% drop) and the bandwidth is increased by less than 1 relative \%. According to eq. (7.10), the LMG optical performance should be significantly affected by a cover layer with a thickness of $20 \mathrm{~nm}$ or more. A simulation of such a layer is shown in figure 7.3 and clearly shows that such a layer indeed starts to reduce the peak reflectivity $(5 \mathrm{pp}$, 11 relative \%) and increase the bandwidth $(>3$ relative \%) significantly. As expected from conditions (7.10) and (7.11) the cover layer indeed has a much stronger effect on peak reflectivity than on bandwidth. Finally, both oxygen $(540 \mathrm{eV})$ and fluorine $(690 \mathrm{eV})$ have absorption edges within the simulated region. As can be seen in figure $7.3 \mathrm{~b}$ peak reflectivity is reduced even further and the bandwidth is slightly increased just above these edges.

Using both scanning and transmission electron microscopy, we determined our contamination layer to be well below $20 \mathrm{~nm}$. Assuming a $5 \mathrm{~nm}$ contamination layer with a composition as determined from the XPS measurements, we determined the optical effect of the contamination layer to result in at 


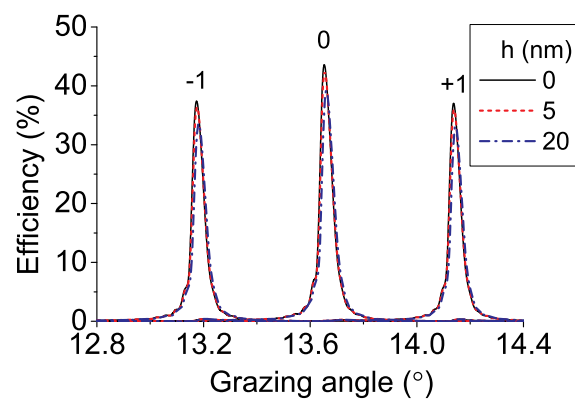

(a)

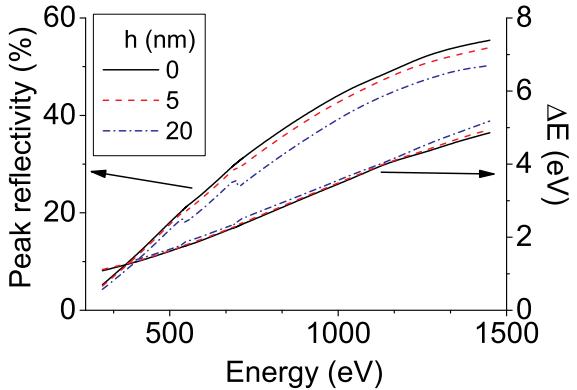

(b)

Figure 7.3: (a) $0^{\text {th }}$ and $\pm 1^{\text {st }}$ order efficiencies for varying SiOF cover layer thicknesses at an incident energy of $1041 \mathrm{eV}$. (b) Energy dependence of peak reflectivity and bandwidth for various cover layer thicknesses.

most a few percent peak reflectivity loss and negligible effects $(<1 \%)$ on the bandwidth. Having investigated sidewall degradation effects, we continue our lifetime study by examining possible structural changes to the ML.

\subsubsection{Thermal annealing}

Patterned ML mirrors are vulnerable to annealing, i.e. structural changes of the ML due to thermal loads. For W/Si ML mirrors, annealing can already start at elevated temperatures of as low as $50^{\circ} \mathrm{C}$ [109]. As LMGs are exposed to temperatures of up to $120^{\circ} \mathrm{C}$ during the fabrication process, they are at a certain risk of annealing. We therefore examined ML annealing as function of both temperature and time. For these annealing experiments, the samples were heated using a hotplate with a temperature accuracy of $0.5^{\circ} \mathrm{C}$ in an air environment. The bi-layer period changes were determined from shifts in the peak positions of Grazing Incidence X-ray Reflectometry (GIXR) measurements as previously discussed in section 2.2.2. Due to the preparation method, all samples were exposed to an unavoidable 2 min bake at $120^{\circ} \mathrm{C}$ before the annealing tests were performed. However, SXR reflectivity measurements performed before and after a similar baking step performed at $150{ }^{\circ} \mathrm{C}$ for 3 mins showed peak reflectivity and bandwidth remained stable to within the measurement accuracy of $1 \%$.

The results of the annealing experiments when varying the temperature are presented in table 7.1. Here, it can be seen that even short exposures to temperatures above $120^{\circ} \mathrm{C}$ quickly results in significant reduction of the bilayer period. As the fabrication process of LMGs requires short exposures to temperatures in the order of $120^{\circ} \mathrm{C}$, the time dependence of the annealing at this temperature was therefore also investigated. Table 7.2 shows that at this 
Table 7.1: Changes in bi-layer period and peak reflectivity due to annealing of a $\mathrm{W} / \mathrm{Si}$ ML mirror after 5 mins at various temperatures measured using $\mathrm{Cu}-K \alpha$ GIXR.

\begin{tabular}{c|c|c|c} 
& \multicolumn{3}{|c}{ Variation in \% of } \\
Temp $\left({ }^{\circ} \mathrm{C}\right)$ & bi-layer period $d$ & $R_{1, \text { peak }}$ & $R_{3, \text { peak }}$ \\
\hline 20 & 0 & 0 & 0 \\
120 & 0.0 & -2.5 & -0.9 \\
200 & -0.2 & -3.4 & -45.7 \\
300 & -0.8 & -5.0 & -95.0
\end{tabular}

temperature, the bi-layer reduction remains very small for significant exposures extending up to several tens of hours. Besides a slight change in the bi-layer period, a reduction in peak efficiency is also clearly seen. This reduction could be caused by a decrease in the optical modulation between the layers in the ML stack due to intermixing as well as thermally induced contamination. In general, the optical effect of short ( $<5$ mins) exposures to temperatures up to $120^{\circ} \mathrm{C}$ is limited to only a few relative percent peak reflectivity loss.

Table 7.2: Change in bi-layer period and peak reflection efficiency of a $\mathrm{W} / \mathrm{Si} \mathrm{ML}$ mirror due to annealing for increasing periods of time at $120^{\circ} \mathrm{C}$. Measurements were carried out using in-situ GIXR at the $\mathrm{Cu}-K \alpha$ energy.

\begin{tabular}{c|c|c|c} 
& \multicolumn{3}{|c}{ Variation in $\%$ of } \\
$\mathrm{t}(\mathrm{hrs})$ & bi-layer period $d$ & $R_{1, \text { peak }}$ & $R_{3, \text { peak }}$ \\
\hline 0 & 0 & 0 & 0 \\
$5 / 60$ & 0.0 & -2.5 & -0.9 \\
10 & -0.05 & -2.4 & -25.1 \\
60 & -0.06 & -2.3 & -27.1
\end{tabular}

Besides heating the substrate during the fabrication process, thermal loading of the ML stack also occurs during the etch step. In this step, the etch species exothermically react with the ML materials and therefore locally deposit heat in the ML structure. Possible annealing due to the etching process was therefore also investigated. As the LMGs were fabricated on samples that also included ML references, the references hence went through the same heating cycles and etching steps. SXR reflectivity measurements from the ML references were therefore compared to measurements of ML samples that were not exposed to the etching steps, referred to as unexposed ML.

Using GIXR measurements, we determined the initial ML mirror to have a $2 \%$ shift in bi-layer period over a distance of $70 \mathrm{~mm}$. Comparing the ML reference and unexposed ML, no additional change in bi-layer period was found within this measurement accuracy. As this accuracy is quite low in comparison to the changes found in tables 7.1-7.2, we examined the reflectivity curve of these samples in more detail by aligning the curves on the maximum peak 
position. It can be seen in figure $7.4 \mathrm{a}$ that the measured reflectivity curves show only minute differences, e.g. the difference in peak reflectivity is less than 0.5 relative $\%$, indicating no significant changes between the unexposed ML and ML reference have occurred. Similar measurements at an incident energy of $525 \mathrm{eV}$ showed the same results.

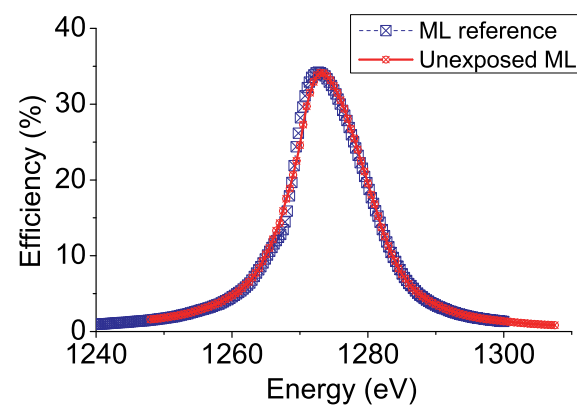

(a)

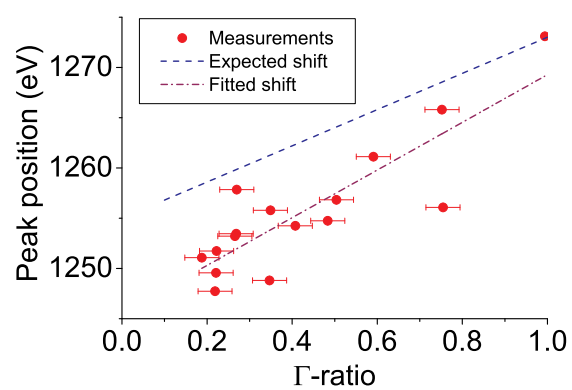

(b)

Figure 7.4: (a) Comparison of measured reflectivity curve of an unexposed ML and the ML reference at the incident energy of $1255 \mathrm{eV}$. (b) Measured (red dots) position of the reflectivity peak for various LMG structures and the ML reference. The linear fit (dashed-dotted purple line) showed a slope of $27 \pm 1 \mathrm{eV} / \Gamma$ compared to an expected value of $18 \mathrm{eV} / \Gamma$ (dashed blue line). The discrepancy was determined to be the result of nonuniformities in the bi-layer period over the sample area.

However, the heat of the exothermic chemical etching may have been localized to the LMG structures without affecting the ML reference. We therefore compared the peak position of the LMGs with the ML reference with as shown in figure 7.4b. Unfortunately, the peak position of LMGs is expected to shift due to the change in average susceptibility for different grating structures $[5,6]$. This shift depends on the lamel-width-to-grating-period ratio $\Gamma$, which can be derived from the generalized Bragg condition for specular reflection (eq. (3.26)) [6]:

$$
\begin{aligned}
& \frac{j \lambda}{2 d}=\sin \Theta_{0}-\frac{\Gamma}{2 \sin \Theta_{0}} . \\
& \quad\left[\operatorname{Re}(\bar{\chi})-\operatorname{Re}\left(\chi_{A}-\chi_{S}\right) \frac{\operatorname{Im}\left(\chi_{A}-\chi_{S}\right)}{\operatorname{Im}(\bar{\chi})} \frac{\sin ^{2}(\pi j \gamma)}{(\pi j)^{2}}\right],
\end{aligned}
$$

where $j$ is the Bragg order of reflection, $\lambda$ is the incident wavelength, $d$ is the bi-layer period, $\Theta_{0}$ is the grazing incidence angle, $\chi$ is the susceptibility of the Absorber and Spacer and $\bar{\chi}$ is the average susceptibility of a bi-layer. The derivative of the generalized Bragg condition to $\Gamma$ was then derived to be:

$$
\Delta \lambda=\left[\operatorname{Re}(\bar{\chi})-\operatorname{Re}\left(\chi_{A}-\chi_{S}\right) \frac{\operatorname{Im}\left(\chi_{A}-\chi_{S}\right)}{\operatorname{Im}(\bar{\chi})} \frac{\sin ^{2}(\pi \gamma)}{\pi^{2}}\right] \cdot \frac{d}{\sin \Theta_{0}} \Delta \Gamma
$$


To calculate the expected peak shift for LMGs, we used the same ML parameters as in section 7.2.1. At the incident energy of $1255 \mathrm{eV}$, this results in $\Delta \lambda \approx 0.015 \Delta \Gamma$ or, equivalently, an slope in energy versus $\Gamma$ of $\approx 18 \Delta \Gamma$. However, a slope of $27 \pm 1 \mathrm{eV} / \Gamma$ was found when fitting the measured peak position as function of the $\Gamma$-ratio. The same relative discrepancy was also found for the measurements at an incident energy of $525 \mathrm{eV}$. We determined the difference between the fitted and expected shift of figure $7.4 \mathrm{~b}$ to correlate with the position of the LMG on the sample area.

As changes to the bi-layer period could again not be measured with sufficient accuracy to determine ML structural changes, we investigated the dependence of LMG optical performance on the grating parameters. Although this comparison showed minor differences of a few relative percent in peak reflectivity, this difference scaled with the number of sidewalls and was nicely explained by the sidewall contamination layer. Changes in optical performance due to the LMG fabrication process were therefore determined to be below the measurement accuracy of $0.1 \mathrm{pp}$ peak reflectivity and $0.1 \mathrm{eV}$ bandwidth.

\subsubsection{Oxygen diffusion}

The sidewalls of patterned W/Si ML mirrors contained large amounts of oxygen as discussed in section 7.2.1, which is known to affect optical performance of Mo/Si multilayer optics used for EUV lithography [97, 100]. Although oxygen diffusion is not expected to occur to a significant degree in our W/Si MLs [85, 110], the fabrication of LMGs does increase the risk of oxygen diffusion. We therefore measured SXR peak reflectivity around the oxygen edge for 3 LMGs with varying grating periods and comparable $\Gamma$-ratios. These measurements were carried out after 23 months of storage in a 1 atm air environment, allowing ample time for diffusion of oxygen into the ML. Figure 7.5 shows the results of these measurements, where the ML reference measurement in figure 7.5a was shifted in peak reflectivity to compensate for peak reflectivity loss due to the grating structure of the LMGs.

Examining figure 7.5a, it can clearly be seen that the dip at $531 \mathrm{eV}$ is only present in the LMG samples. The depth of this dip was plotted against the sidewall-to-lamel-width ratio $(2 h /(\Gamma D))$, showing a clear dependence on the grating structure and, hence, the number of sidewalls the soft x-rays pass through. From the dip position, we determined this dip was caused by tungsten oxides $[107,111]$. Using the simulations of the previous section, we determined a $3 \mathrm{~nm}$ tungsten oxide layer on the sidewalls is required to explain the depth of the dip. This layer thickness was compared to the layer thickness determined from the XPS measurements. In those measurements, we found an atomic concentration of approximately $10 \%$ tungsten oxide. Assuming a sample depth for the XPS of $5 \mathrm{~nm}$, this results in a $0.5 \mathrm{~nm}$ tungsten oxide layer. However, the simulations used purely theoretical cross-sections from ref. [23] and the XPS measurements assumed a homogenous sidewall layer as well as a $5 \mathrm{~nm}$ sample depth, resulting in an estimation accuracy of the tungsten layer thickness of 


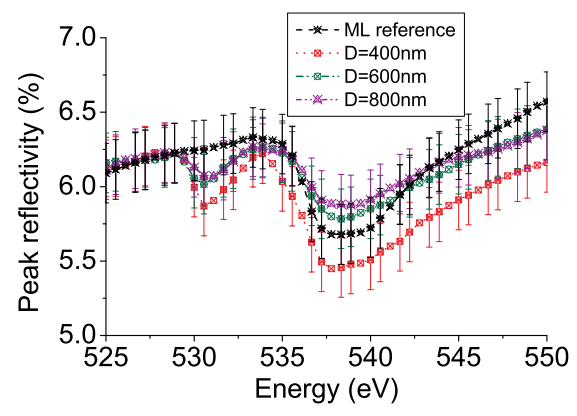

(a)

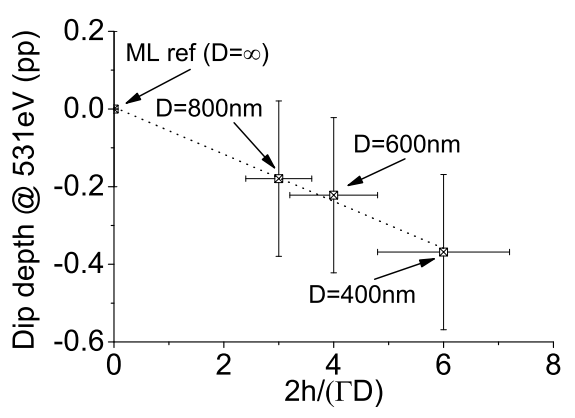

(b)

Figure 7.5: (a) SXR peak reflectivity around the oxygen edge for several LMGs with varying grating periods and similar $\Gamma$-ratios. The ML reference measurement also showed a dip at $538 \mathrm{eV}$, indicating this is not caused by the LMG structures. (b) Dip depth at $531 \mathrm{eV}$ due to tungsten oxide as function of the sidewall-to-lamel-width ratio.

at best a few nm. The estimated layers thicknesses using both methods were therefore considered to be in reasonable agreement, showing a layer thickness of a few nm. The reflectivity measurements clearly showed the tungsten oxide remained localized close to the sidewalls even after 23 months storage and, hence, no oxygen diffusion was measured.

Silicon oxide was also found in the XPS measurements and should show up in figure 7.5a as a dip at $533 \mathrm{eV}$. However, this dip is not visible in these measurements. Assuming a detection limit of $0.1 \%$ and using again the simulations of the previous section, the maximum thickness of the silicon oxide layer on the sidewall was determined to be $3 \mathrm{~nm}$. In comparison, from the XPS measurements a $1 \mathrm{~nm}$ silicon oxide layer would be expected. Silicon oxide was thus only found in negligible amounts on the sidewall and did not appear to diffuse into the ML stack. Finally, the dip at $538 \mathrm{eV}$ corresponds to oxygen compounds with minimal shifts in binding energy, such as water or various carbon oxide combinations [107, 111]. However, this dip was present in all measurements, including the ML reference, and was therefore concluded not to be caused by the LMG structure.

\subsubsection{Stability of SXR reflectivity over time}

To determine the effect of storage degradation processes, specifically sidewall contamination and oxygen diffusion, we investigated the stability of the optical performance of LMGs over a storage period of 23 months. The bandwidth and peak reflectivity were compared to earlier measurements, as shown in figure 7.6. Here, it can easily be seen that the optical performance of the LMGs remained 
stable to within measurement accuracy $\left(R_{\text {peak }}<0.1 \%\right.$ and $\left.\Delta E<0.1 \mathrm{eV}\right)$ during this period. The peak position of the different LMGs was also compared before and after storage (not shown) to investigate changes in the bi-layer period. The peak position also remained stable to within the measurement accuracy of $0.1 \mathrm{eV}$.

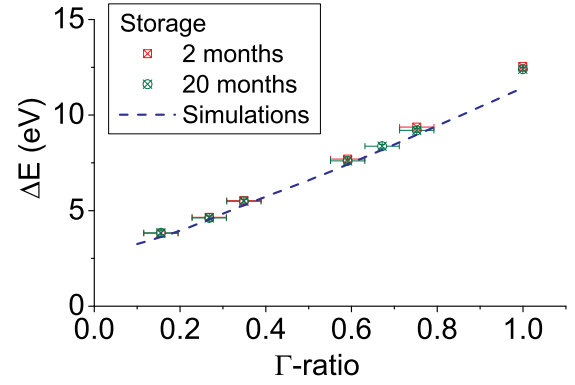

(a)

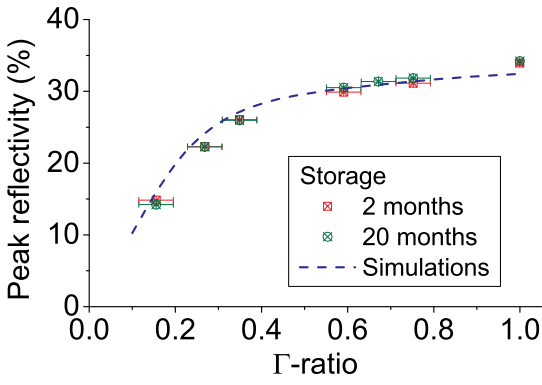

(b)

Figure 7.6: Comparison of (a) FWHM bandwidth and (b) peak reflectivity of W/Si LMGs at an incident energy of $1255 \mathrm{eV}$ after 18 months of storage in $1 \mathrm{~atm}$ air environment.

\subsection{Capping layers}

Although our W/Si LMGs did not show any significant signs of degradation over a 23 month storage period, radiation induced process [95, 97, 100] were not investigated in this work. A preliminary investigation into the use of capping layers on LMG structures to reduce degradation was performed.

\subsubsection{Possible capping materials}

Similar to refs. [100, 112] suitable capping layer materials for the protection of patterned ML structures can be determined based on thermal and chemical stability as well as optical properties. Although most of such arguments also hold for etched ML structures, there are a few significant differences between capping layers for conventional and etched ML mirrors. Most notably, the capping layer must be placed on the sidewalls, which means the chemical stability with both ML materials has to be determined. Furthermore, the incident radiation generally passes through many sidewalls in patterned MLs, each covered with a capping layer, such that the total thickness of capping material the radiation passes through is much larger for a patterned as compared to a conventional ML mirror. 
From refs. [100, 112], several chemically suitable materials for capping layers can be determined, such as ruthenium, zirconium and silicon nitride. Using the simulations of cover layers as discussed in section 7.2.1, the effect of various capping layer materials on the optical performance of LMGs was investigated assuming a $5 \mathrm{~nm}$ thick layer. The results of these simulations are shown in figure 7.7, where it can be seen that silicon nitride has the smallest effect on bandwidth and peak reflectivity. Furthermore, it can also be seen that several materials have absorption edges in the wavelength range of interest, most notable of which is chromium. From this analysis, silicon nitride was concluded to be the most suitable capping layer material as this material has a minimal effect on LMG optical performance and is both chemically and thermally stable.

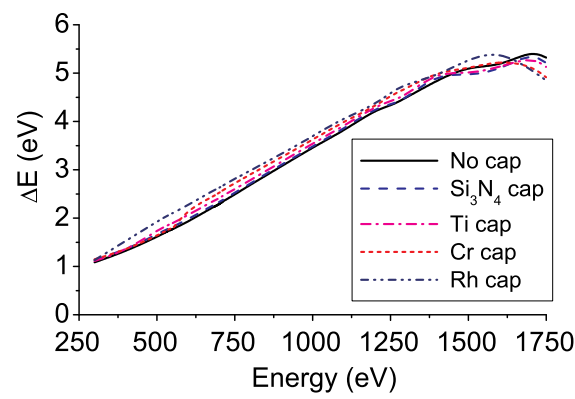

(a)

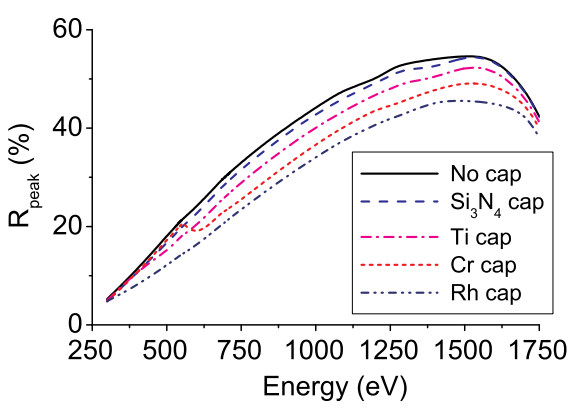

(b)

Figure 7.7: Simulations of (a) FWHM bandwidth and (b) peak reflectivity for various capping layer materials with a constant thicknesses of $5 \mathrm{~nm}$. The simulated LMG is the same as used for figure 7.3.

\subsubsection{Preliminary silicon nitride deposition tests}

Because silicon nitride holds good promise for use as capping layer, we carried out some preliminary silicon nitride deposition tests. These were performed using Plasma Enhanced Chemical Vapor Deposition. The resulting capping layers were analyzed using SEM and EF-TEM to determine composition and coverage of the deposited layer on the grating topography. In CVD, one or more volatile precursors react or dissociate to produce the required deposition material. The deposition of silicon nitride in CVD is performed using a combination of silane in nitrogen $\left(2 \% \mathrm{SiH}_{4} / \mathrm{N}_{2}\right)$ and ammonia $\left(\mathrm{NH}_{3}\right)$ following:

$$
3 \mathrm{SiH}_{4}+4 \mathrm{NH}_{3} \rightarrow \mathrm{Si}_{3} \mathrm{~N}_{4}+12 \mathrm{H}_{2}
$$

at typical process temperatures of well over $1000^{\circ} \mathrm{C}$. The chemical reaction rates can be enhanced by using a plasma to dissociate the precursors, the so-called Plasma Enhanced CVD (PE-CVD) process. This allows the use of 
much lower temperatures to avoid annealing of the ML structure. Although refs. [95, 113] have nitridated silicon layers at room temperature using $100 \mathrm{eV}$ $\mathrm{N}^{+}$ions from a Kaufman source, such processes are highly directional and cannot be easily used to deposit material on the sidewalls of etched ML mirrors.

For our deposition tests, we used a PE-CVD system (Oxford 80) that is able to deposit silicon nitride at temperatures as low as $100^{\circ} \mathrm{C}$. This temperatures is well below the maximum allowable temperature of $120^{\circ} \mathrm{C}$ to prevent the annealing of ML structures as discussed in section 7.2.2. For these tests, we also used structured silicon samples that were more readily available than LMGs and, as an added benefit, had larger scallops and therefore more clearly showed the uniformity and coverage of the deposition process.

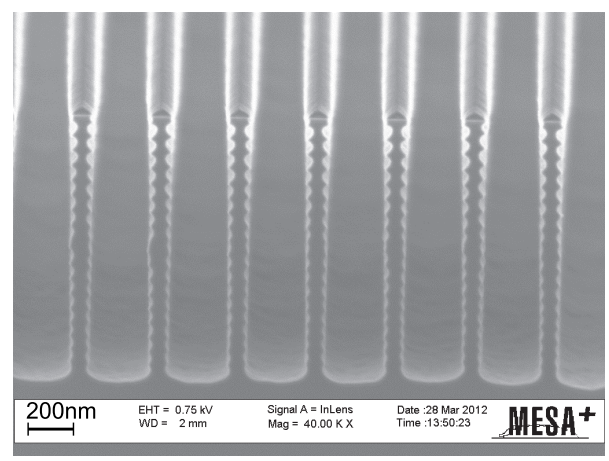

(a)

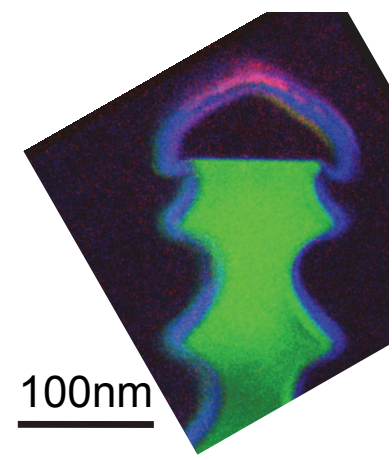

(b)

Figure 7.8: (a) Preliminary silicon nitride deposition test on silicon nanostructures using 1 min deposition time. (b) EF-TEM analysis of the resulting capping layer, where silicon is indicated by green, oxygen is indicated by blue and nitrogen is indicated by red. The image clearly shows the capping layer consists mostly of silicon oxide and only contains nitrogen at the very top part of the structure.

Figure 7.8a shows a silicon sample coated with a silicon nitride capping layer and imaged using SEM. The figure clearly shows the etched grating structure with some remaining mask material at the top of the structure. However, the deposited silicon nitride layer is barely visible as the light haze in this image. This structure was therefore also analyzed using EF-TEM as shown in figure 7.8b. Here, the spatial distribution of silicon is shown in green, oxygen in blue and nitrogen in red. It can be seen that a capping layer with a thickness in the order of $5 \mathrm{~nm}$ is present at the sidewalls. The layer is clearly less thick inside the lamel, showing the process appears to be insufficiently directional.

From the image, the composition of the layer can be seen to consist mostly of oxygen and silicon, although the silicon in the layer is poorly visible due to the high concentration of silicon inside the lamel. Furthermore, it can be seen that only a small amount of nitrogen is present in the layer. It was expected 
that although the plasma dissociated the silane and ammonia, the temperature was insufficient to nitridate the deposited silicon. This resulted in a deposition of a pure silicon layer which, when exposed to air, immediately oxidized and resulted in a layer of silicon oxide instead of the desired silicon nitride.

\subsection{Conclusions}

The lifetime of patterned multilayer (ML) mirrors is very important for the usability of these elements in practical applications. We therefore investigated various degradation processes that could reduce this lifetime and that could occur during fabrication and storage during 23 months in 1 atm air environment. This study was performed for Lamellar Multilayer Gratings (LMGs), which are optical elements used for soft x-ray spectroscopy, fabricated in W/Si ML mirrors. The results are expected to also be applicable to other optical elements fabricated in W/Si ML mirrors that were fabricated using the same fabrication process and stored under similar conditions.

We first investigated sidewall contamination as a surface phenomenon that increases the absorption and hence reduces peak reflectivity of LMGs. The composition of the sidewall contamination layer was measured with x-ray photoelectron spectroscopy, showing a significant desorption of fluorine during storage. An extension to our Coupled Waves Approach (CWA) simulation method was derived to simulate the optical effect of this contamination layer. Criteria for acceptable contamination layer thicknesses for which peak reflectivity and bandwidth are not significantly affected were also derived. This analysis revealed layers less than $5 \mathrm{~nm}$ with the measured composition had a limited effect of at most a few relative percent peak reflectivity loss.

Degradation due to structural changes to the ML caused by thermal annealing and oxygen diffusion were investigated next. This analysis showed the ML was stable for long period (>60 hrs) at $120^{\circ} \mathrm{C}$, but quickly deteriorated at higher temperatures. Oxygen diffusion was investigated using SXR reflectivity measurements around the oxygen edge, showing oxides were still localized to the sidewall even after 23 months storage. To determine the overall effect of storage, we measured the stability of SXR reflectivity over an extended storage period of 18 months. This analysis showed the optical performance of LMGs to remain stable to within measurement accuracy during this period.

Though not studied here, degradation is expected to be enhanced under irradiation with soft x-ray photons. We therefore investigated various possible capping layer materials to protect the LMG from degradation and during carbon cleaning processes. Suitable capping layers were theoretically studied, showing silicon nitride to be the preferred candidate. Preliminary deposition tests of silicon nitride were performed using plasma enhanced chemical vapor deposition at the low temperatures required to prevent annealing of the ML. However, these tests showed the resulting layer was poorly nitridated and did not sufficiently cover the LMG topography for use as a capping layer. 


\section{8 \\ Conclusions}

The ability to identify and quantify chemical elements in products is very important to ensure, for instance, that steel and concrete are of sufficient quality to be used for construction or that there are no toxic contaminants in our food or drinks. A major improvement in chemical element identification was obtained after the discovery of x-rays. Elements could now be uniquely and non-destructively identified by analyzing the fluorescent x-ray spectrum emitted after a sample had been irradiated with high energy x-rays, which is the basic principle of x-ray fluorescence spectroscopy. To analyze the fluorescence spectrum, an optical element is required that separates x-rays with different energies. In the soft x-ray spectral range, which is of importance for identifying lighter elements, such elements are often multilayer (ML) mirrors that work on the principle of Bragg reflection. Unfortunately, the spectral resolution of ML mirrors is inherently limited in this range by the absorption of the fluorescent x-rays that need to be analyzed. The spectral resolution of ML mirrors can be improved by fabricating a grating into the ML stack, resulting in a so-called Lamellar Multilayer Grating (LMG). However, the usability of LMGs has been limited due to challenges in simulation, which limited the understanding of the LMG working principles, and fabrication, which limited the grating dimensions and ML stack height of LMGs. In this thesis, a simulation and fabrication method were investigated which overcame the aforementioned limitations and improved the usability of LMGs in practical applications.

We first derived a Coupled Waves Analysis (CWA) to simulate LMG optical performance in terms of bandwidth and peak reflectivity. This CWA describes the change in amplitude of the incident and exiting waves as function of position in the ML stack. Compared to previously used simulation methods, this CWA significantly reduces computation time, can simulate various non-ideal lamel shapes and allows for improved understanding of LMG operating principles. 
Based on this CWA, we identified an optimal LMG operating regime, referred to as single-order operation, in which the incident beam excites only a single diffraction order and, hence, is not affected by diffraction losses. For LMGs operating in single-order, the optical performance is largely determined by the lamel-width-to-grating-period ratio $\Gamma$. The resolution of an LMG was derived to scale with $1 / \Gamma$ in comparison to a conventional ML mirror. LMG peak reflectivity was determined to be as high as that of a conventional ML mirror if the number of bi-layers $N$ was also increased by $1 / \Gamma$.

The fabrication of single-order operating LMGs requires the patterning of nanoscale structures in MLs made of up to hundreds of thin bi-layers with a period of a few nm. These bi-layers consist of, at least, two materials of which one is generally a high-Z material. Although e-beam lithography can be used to define LMG structures, this is a time-consuming and costly process. Pattern transfer using Reactive Ion Etching (RIE) is generally insufficient accurate or reproducible. We therefore investigated the patterning of ML mirrors using UV-NanoImprint Lithography and Bosch Deep Reactive Ion Etching (DRIE). This process provides accurate and reproducible fabrication of LMGs with submicron periods over tens of square $\mathrm{mm}^{2}$ area in an economic manner. The ML structure itself was used to achieve optimal pattern transfer. In particular, the high- $Z$ layer in the ML stack was used to suppress scallop formation and allow for low tapers with minimal grating structure dependence. Although this fabrication study was performed for the patterning of grating structures in W/Si ML mirrors, the approach and results are generally applicable to ML mirrors consisting of materials that react volatile with fluorine. Such MLs include $\mathrm{Mo} / \mathrm{Si}$ for EUV lithography and $\mathrm{Mo} / \mathrm{B}_{4} \mathrm{C}$ for spectroscopy. However, the activation energy of the reactions with fluorine as well as possible compound formation in the ML structure can significantly affect etching. The minimal dependence on grating dimensions and low tapers of the derived process are highly beneficial for the fabrication of optical elements with varying feature dimensions, such as zoneplates.

Reflectivity of single-order LMGs for the SXR spectral range was measured and was found to be in good agreement with our CWA. Single-order LMGs had limited diffraction losses, which was evident from the absence of any significant secondary structures besides the main Bragg peak and a corresponding increase of the peak reflectivity with respect to a multi-order LMG having the same $\Gamma$ ratio. A record value of spectral resolution improvement was achieved using single-order operation, which is currently a factor of 3.8 with regard to the unpatterned ML mirror. Excitation of higher diffraction orders in single-order operation was easily measured by varying the detector angle and was also in good agreement with our CWA. Our CWA can thus also be used to investigate the use of higher diffraction orders from LMGs for various applications. Finally, we determined non-uniformities of the bi-layer period in the depth of the initial ML are currently limiting LMG performance. Further improvements of LMG performance can therefore be obtained by increasing the ML deposition process.

Current technologies for ML deposition and grating fabrication actually 
often do not achieve sufficient accuracy to fulfill the semi-infinite ML approximation. However, this approximation is often used for ML and LMG design. Assuming the semi-infinite approximation, LMGs can be designed by first optimizing the absorber-thickness-to-bi-layer period $\gamma$ of the ML to achieve maximum peak reflectivity and subsequently reducing the bandwidth to any desired value using the lamel-width-to-grating-period ratio $\Gamma$. In particular for LMGs with low $\Gamma$-ratio's, this approximation is often not valid. We therefore derived a criterion for the transition from the semi-infinite to finite ML case based on the SXR penetration depth. To determine the effect of a finite ML stack, simulations of LMG optical performance comparing a semi-infinite and finite ML stacks were performed. For the finite ML stack, the minimal achievable bandwidth and maximum peak reflectivity are limited by the number of bilayers in the ML stack. Optical performance can then be further tailored using the ML $\gamma$-ratio. A lower $\gamma$-ratio will result in slightly narrower bandwidths at the expense of peak reflectivity. If slightly higher bandwidths can be accepted, higher $\gamma$-ratios should be used to minimize peak reflectivity loss.

Finally, we investigated degradation processes of patterned ML optics as this can reduce the lifetime of such elements, which is important for their usability in practical applications. In this work, we focused on degradation during fabrication and storage during 23 months in a 1 atm air environment. The surface phenomenon of sidewall contamination showed a clear desorption of fluorine, but limited optical effect for contamination layers with a thickness below $5 \mathrm{~nm}$. Internal ML structural changes due to thermal annealing or oxygen diffusion were not seen within measurement accuracy. The SXR reflectivity also remained stable over an extended storage period of 18 months within measurement accuracy. Radiation induced degradation processes, including surface photochemistry, need to be investigated separately. An investigation of possible capping layer materials was performed showing silicon nitride to be the preferred candidate. Preliminary deposition tests of silicon nitride were performed using PE-CVD, but the resulting layer was poorly nitridated and did not sufficiently cover the LMG topography for use as a capping layer. Although the degradation study was performed for the specific case of LMGs, the results are expected to also be applicable to other Bragg-Fresnel optics fabricated in W/Si ML mirrors using the same fabrication process and stored under similar conditions. 



\section{References}

[1] J. A. C. Broekaert, Analytical Atomic Spectrometry with Flames and Plasmas. Wiley, 2006.

[2] H. G. J. Moseley, "XCIII. The high-frequency spectra of the elements," Philosophical Magazine Series 6, vol. 26, no. 156, pp. 1024-1034, 1913.

[3] H. G. J. Moseley, "LXXX. The high-frequency spectra of the elements. Part II," Philosophical Magazine Series 6, vol. 27, no. 160, pp. 703-713, 1914.

[4] I. V. Kozhevnikov and A. V. Vinogradov, "Basic formulae of XUV multilayer optics," Physica Scripta T, vol. 17, pp. 137-145, 1987.

[5] I. V. Kozhevnikov, R. van der Meer, H. M. J. Bastiaens, K.-J. Boller and F. Bijkerk, "High-resolution, high-reflectivity operation of lamellar multilayer amplitude gratings: identification of the single-order regime," Optics Express, vol. 18, no. 15, pp. 16234-16242, 2010.

[6] I. V. Kozhevnikov, R. van der Meer, H. M. J. Bastiaens, K.-J. Boller and F. Bijkerk, "Analytic theory of soft x-ray diffraction by lamellar multilayer gratings," Optics Express, vol. 19, no. 10, pp. 9172-9184, 2011.

[7] R. van der Meer, I. Kozhevnikov, B. Krishnan, J. Huskens, P. Hegeman, C. Brons, B. Vratzov, B. Bastiaens, K. Boller and F. Bijkerk, "Singleorder operation of lamellar multilayer gratings in the soft x-ray spectral range," AIP Advances, vol. 3, no. 1, pp. 012103-1/7, 2013.

[8] M. O. Krause and J. H. Oliver, "Natural widths of atomic $K$ and $L$ levels, $K \alpha$ X-ray lines and several KLL Auger lines," Journal of Physical and Chemical Reference Data, vol. 8, pp. 329-338, 1979.

[9] R. A. M. Keski-Kuha, "Layered synthetic microstructure technology considerations for the extreme ultraviolet," Applied Optics, vol. 23, no. 20, pp. 3534-3537, 1984.

[10] J.-M. André, A. Sammar, C. K. Malek, P. Troussel, S. Bac, R. Barchewitz, B. Pardo, H. Berrouane, T. Moreno, F. R. Ladan, R. Rivoira and D. Schirmann, "Multilayer gratings for the soft x-ray region (invited)," Review of Scientific Instruments, vol. 63, no. 1, pp. 1399-1403, 1992. 
[11] A. I. Erko, B. Vidal, P. Vincent, Y. A. Agafonov, V. V. Martynov, D. V. Roschupkin and M. Brunel, "Multilayer gratings efficiency: numerical and physical experiments," Nuclear Instruments and Methods in Physics Research Section A, vol. 333, no. 2-3, pp. 599-606, 1993.

[12] R. Benbalagh, J.-M. André, R. Barchewitz, P. Jonnard, G. Julié, L. Mollard, G. Rolland, C. Rémond, P. Troussel, R. Marmoret and E. O. Filatova, "Lamellar multilayer amplitude grating as soft-x-ray Bragg monochromator," Nuclear Instruments and Methods in Physics Research Section A, vol. 541, pp. 590-597, 2005.

[13] S. Andreev, A. Akhsakhalyan, M. Bibishkin, N. Chkhalo, S. Gaponov, S. Gusev, E. Kluenkov, K. Prokhorov, N. N. Salashchenko, F. Schafers and S. Zuev, "Multilayer optics for XUV spectral region: technology fabrication and applications," Central European Journal of Physics, vol. 1, no. 1, pp. 191-209, 2003.

[14] E. Hecht, Optics. Pearson education, San Fransisco, $4^{\text {th }}$ ed., 2002.

[15] E. Spiller, Soft X-Ray Optics. SPIE Press monograph, SPIE Optical Engineering Press, 1994.

[16] M. J. H. Kessels, Interfaces in soft x-ray multilayer mirrors. PhD thesis, University of Twente, 2005.

[17] S. Bruijn, Diffusion phenomena in chemically stabilized multilayer structures. PhD thesis, University of Twente, 2011.

[18] I. V. Kozhevnikov and A. V. Vinogradov, "Multilayer x-ray mirrors," Journal of Russian Laser Research, vol. 16, no. 4, pp. 343-385, 1995.

[19] M. S. Bibishkin, N. I. Chkhalo, A. A. Fraerman, A. E. Pestov, K. A. Prokhorov, N. N. Salashchenko and Y. A. Vainer, "Ultra-short period X-ray mirrors: Production and investigation," Nuclear Instruments and Methods in Physics Research Section A, vol. 543, no. 1, pp. 333 - 339, 2005.

[20] M. Born and E. Wolf, Principles of optics. Electromagnetic theory of propagation, interference and diffraction of light. Oxford: Pergamon Press, 1980, 6 ${ }^{\text {th }}$ ed., 1980.

[21] C. Huygens, "Traité de la Lumiere," 1678. (Leyden, 1690).

[22] A. Fresnel, "Mémoire sur la diffraction de la lumière," Annales de chimie et de physique, vol. 1, pp. 239-281, 1816.

[23] B. L. Henke, E. M. Gullikson and J. C. Davis, "X-Ray Interactions: Photoabsorption, Scattering, Transmission, and Reflection at E $=50$ 30,000 eV, z=1-92," Atomic Data and Nuclear Data Tables, vol. 54, no. 2, pp. $181-342,1993$. 
[24] R. D. Deslattes, E. G. Kessler, P. Indelicato, L. de Billy, E. Lindroth and J. Anton, "X-ray transition energies: new approach to a comprehensive evaluation," Reviews of Modern Physics, vol. 75, pp. 35-99, 2003.

[25] D. Attwood, Soft x-rays and Extreme Ultraviolet Radiation - Principles and Applications. Cambridge University Press, Cambridge, 1999.

[26] R. B. Hoover, D. L. Shealy, B. R. Brinkley, P. C. Baker, T. W. Barbee, Jr. and A. B. C. Walker II, "Development of the water window imaging x-ray microscope utilizing normal incidence multilayer optics," Optical Engineering, vol. 30, no. 8, pp. 1086-1093, 1991.

[27] S. Werner, S. Rehbein, P. Guttmann, S. Heim and G. Schneider, "Towards high diffraction efficiency zone plates for x-ray microscopy," $M i$ croelectronic Engineering, vol. 87, no. 5-8, pp. 1557 - 1560, 2010. The $35^{\text {th }}$ International Conference on Micro- and Nano-Engineering (MNE).

[28] M. Ahn, Fabrication of Critical-Angle Transmission Gratings for High Efficiency X-ray Spectroscopy. PhD thesis, Massachusetts Institute of Technology, 2009.

[29] A. G. Michette, S. J. Pfauntsch, S. Sahraei, M. Shand, G. R. Morrison, D. Hart, B. Vojnovic, T. Stevenson, W. Parkes, C. Dunare, R. Willingale, C. Feldman, T. Button, D. Zhang, D. Rodriguez-Sanmartin and H. Wang, "Smart x-ray optics," Journal de Physique: Conference Series, vol. 186, no. 1, p. 012067, 2009.

[30] H. C. Kang, H. Yan, R. P. Winarski, M. V. Holt, J. Maser, C. Liu, R. Conley, S. Vogt, A. T. Macrander and G. B. Stephenson, "Focusing of hard x-rays to 16 nanometers with a multilayer Laue lens," Applied Physics Letters, vol. 92, no. 22, p. 221114, 2008.

[31] "European Synchrotron Radiation Facility." 6 rue Jules Horowitz, BP 220, 38043 Grenoble Cedex 9, France.

[32] C. Morawe, P. Pecci, J.-C. Peffen and E. Ziegler, "Design and performance of graded multilayers as focusing elements for x-ray optics," Review of Scientific Instruments, vol. 70, no. 8, pp. 3227-3232, 1999.

[33] C. Morawe, E. Ziegler, J.-C. Peffen and I. V. Kozhevnikov, "Design and fabrication of depth-graded x-ray multilayers," Nuclear Instruments and Methods in Physics Research Section A, vol. 493, no. 3, pp. 189 - 198, 2002.

[34] "BESSYII synchrotron facility." Albert-Einstein-Str. 15, 12489 Berlin, Germany.

[35] A. I. Erko, M. Idir, T. Krist and A. G. Michette, eds., Modern developments in x-ray and Neutron optics. Berlin: Springer Berlin / Heidelberg, 2008. 
[36] G. Pareschi, V. Cotroneo, D. Spiga, D. Vernani, M. Barbera, M. A. Artale, A. Collura, S. Varisco, G. Grisoni, G. Valsecchi and B. Negri, "Astronomical soft x-ray mirrors reflectivity enhancement by multilayer coatings with carbon overcoating," in UV and Gamma-Ray Space Telescope Systems, vol. 5488 of Proc. of SPIE, pp. 481-491, 2004.

[37] K. K. Madsen, F. E. Christensen, C. P. Jensen, E. Ziegler, W. W. Craig, K. S. Gunderson, J. E. Koglin and K. Pedersen, "X-ray study of W/Si multilayers for the HEFT hard x-ray telescope," in Optics for EUV, $X$ Ray, and Gamma-Ray Astronomy, vol. 5168 of Proc. of SPIE, pp. 41-52, 2004 .

[38] Z. Wang, J. Zhu, R. Chen, J. Xu, F. Wang, Z. Zhang, W. Wu, L. Liu, H. Zhang, D. Xu, H. Jiang, L. Chen, H. Zhou, T. Huo, M. Cui and Y. Zhao, "Extreme ultraviolet multilayer mirrors for astronomical observation," in Sixth International Conference on Thin Film Physics and Applications, vol. 6984 of Proc. of SPIE, pp. 698433-1/6, 2008.

[39] A. E. Yakshin, R. W. E. van de Kruijs, I. Nedelcu, E. Zoethout, E. Louis, F. Bijkerk, H. Enkisch and S. Müllender, "Enhanced reflectance of interface engineered Mo/Si multilayers produced by thermal particle deposition," in Emerging Lithographic Technologies XI, vol. 6517 of Proc. of SPIE, p. 65170I, 2007.

[40] F. van Goor, F. Bijkerk, A. J. R. van den Boogaard and R. van der Meer, "Spectral filter for splitting a beam with electromagnetic radiation having wavelengths in the extreme ultraviolet (euv) or soft x-ray (soft x) and the infrared (ir) wavelength range." International patent WO2012/023853, 2010. Priority date: 18-08-2010; Publication date: 23-02-2012.

[41] V. V. Medvedev, A. J. R. van den Boogaard, R. van der Meer, A. E. Yakshin, E. Louis, V. M. Krivtsun, A. M. Yakunin and F. Bijkerk, "Infrared phase-shift filtering for Extreme UltraViolet multilayer Bragg reflectors." In preparation.

[42] F. Scholze, M. Krumrey, P. Müller and D. Fuchs, "Plane grating monochromator beamline for VUV radiometry," Review of Scientific Instruments, vol. 65, no. 10, pp. 3229-3232, 1994.

[43] D. Fuchs, M. Krumrey, P. Müller, F. Scholze and G. Ulm, "High precision soft x-ray reflectometer," Review of Scientific Instruments, vol. 66, no. 2, pp. 2248-2250, 1995.

[44] F. Scholze, B. Beckhoff, G. Brandt, R. Fliegauf, A. Gottwald, R. Klein, B. Meyer, U. D. Schwarz, R. Thornagel, J. Tuemmler, K. Vogel, J. Weser and G. Ulm, "High-accuracy EUV metrology of PTB using synchrotron radiation," in Metrology, Inspection, and Process Control for Microlithography XV, vol. 402 of Proc. of SPIE, pp. 402-413, 2001. 
[45] F. Scholze, J. Tümmler and G. Ulm, "High-accuracy radiometry in the EUV range at the PTB soft x-ray beamline," Metrologia, vol. 40, no. 1, p. S224, 2003.

[46] J. Daillant and A. Gibaud, X-ray and Neutron Reflectivity: Principles and Applications. Lecture Notes in Physics, Springer, 2008.

[47] K. N. Stoev and K. Sakurai, "Review on grazing incidence x-ray spectrometry and reflectometry," Spectrochimica Acta Part B: Atomic Spectroscopy, vol. 54, no. 1, pp. 41-82, 1999.

[48] D. L. Windt, "IMD-Software for modeling the optical properties of multilayer films," Computers in physics, vol. 12, pp. 360-370, 1998.

[49] P. E. Mix, Introduction to Nondestructive Testing: A Training Guide. John Wiley \& Sons, 2005.

[50] L. Reimer and P. W. Hawkes, Scanning Electron Microscopy: Physics of Image Formation and Microanalysis. Springer Series in Optical Sciences, Springer, 2010.

[51] L. Reimer and H. Kohl, Transmission Electron Microscopy: Physics of Image Formation. Springer Series in Optical Sciences, Springer, 2008.

[52] P. van der Heide, X-ray Photoelectron Spectroscopy: An introduction to Principles and Practices. Wiley, 2011.

[53] J. F. Watts, "X-ray photoelectron spectroscopy," Vacuum, vol. 45, no. 67, pp. 653 - 671, 1994. Special Issue Surface Science Techniques.

[54] B. Vidal, P. Vincent, M. Neviere and P. Dhez, "Thin films and gratings Theories to optimize the high reflectivity of mirrors and gratings for x-ray optics," in Applications of Thin Film Multilayered Structures to Figured X-Ray Optics, vol. 563 of Proc. of SPIE, pp. 142-149, 1985.

[55] S. T. Peng, "Rigorous formulation of scattering and guidance by dielectric grating waveguides: general case of oblique incidence," Journal of the Optical Society of America A, vol. 6, no. 12, pp. 1869-1883, 1989.

[56] A. Coves, B. Gimeno, J. Gil, M. V. Andres, A. A. Sanblas and V. E. Boria, "Full-Wave Analysis of Dielectric Frequency-Selective Surfaces Using a Vectorial Modal Method," IEEE Transactions on antennas and propagation, vol. 52, pp. 2091-2099, 2004.

[57] L. I. Goray, "Numerical analysis of the efficiency of multilayer-coated gratings using integral method," Nuclear Instruments and Methods in Physics Research Section A, vol. 536, pp. 211-221, 2005. 
[58] A. Sammar, M. Ouahabi, R. Barchewitz, J.-M. André, R. Rivoira, C. K. Malek, F. R. Ladan and P. Guérin, "Theoretical and experimental study of soft x-ray diffraction by a lamellar multilayer amplitude grating," Journal of Optics, vol. 24, pp. 37-41, 1993.

[59] A. Sammar, J.-M. André and B. Pardo, "Diffraction and scattering by lamellar amplitude multilayer gratings in the X-UV region," Optics Communications, vol. 86, pp. 245-254, 1991.

[60] K. Krastev, J.-M. André and R. Barchewitz, "Further applications of a recursive modal method for calculating the efficiencies of X-UV multilayer gratings," Journal of the Optical Society of America A, vol. 13, pp. 20272033, 1996.

[61] L. I. Goray and J. F. Seely, "Wavelength separation of plus and minus orders of soft-x-ray-EUV multilayer-coated gratings at near-normal incidence," in Optics for EUV, X-Ray, and Gamma-Ray Astronomy II, vol. 5900 of Proc. of SPIE, pp. 81-91, 2005.

[62] V. V. Martynov, B. Vidal, P. Vincent, M. Brunel, D. V. Roschupkin, Y. A. Agafonov, A. I. Erko and A. Yuakshin, "Comparison of modal and differential methods for multilayer gratings," Nuclear Instruments and Methods in Physics Research Section A, vol. 339, pp. 617-625, 1994.

[63] R. Benbalagh, Monochromateurs Multicouches à bande passante étroite et à faible fond continu pour le rayonnement $X-U V$. PhD thesis, Université Pierre et Marie Curie, 2003.

[64] T. Jannson, G. Savant and L. Wang, "Extreme-ultraviolet Bragg holographic structures: theory and experiments," Journal of the Optical Society of America A, vol. 8, no. 10, pp. 1587-1590, 1991.

[65] D. J. Griffiths, Introduction to Electrodynamics. Benjamin Cummings, $3^{\text {rd }}$ ed., 1998.

[66] R. Petit, Electromagnetic theory of gratings. Berlin: Springer-Verlag, 1980.

[67] A. V. Vinogradov and B. Y. Zeldovich, "X-ray and far UV multilayer mirrors: principles and possibilities," Applied Optics, vol. 16, no. 1, pp. 8993, 1977.

[68] I. V. Kozhevnikov, "General laws of x-ray reflection from rough surfaces: I. Optical theorem and energy conservation law," Crystallography Reports, vol. 55, pp. 539-545, 2010.

[69] D. K. G. de Boer, "X-ray reflection and transmission by rough surfaces," Physical Review B, vol. 51, pp. 5297-5305, 1995. 
[70] I. V. Kozhevnikov, "General laws of x-ray reflection from rough surfaces: II. Conformal Roughness," Crystallography Reports, vol. 57, pp. 490-498, 2011.

[71] P. Jonnard, K. Le Guen, J.-M. André, J.-R. Coudevylle and N. Isac, “An etched multilayer as a dispersive element in a curved - crystal spectrometer: implementation and performance," Journal of X-ray Spectrometry, 2012.

[72] "Xenocs SA." rue Francois Blumet, F-38360 Sassenage, France.

[73] M. Colburn, A. Grot, M. Amistoso, B. J. Choi, T. Bailey, J. Ekerdt, S. V. Sreenivasan, J. Hollenhorst and C. G. Willson, "Step and Flash Imprint Lithography for sub-100nm Patterning," in Emerging Lithographic Technologies IV, vol. 3997 of Proc. of SPIE, pp. 453-457, 2000.

[74] R. van der Meer, B. Krishnan, I. V. Kozhevnikov, M. J. de Boer, B. Vratzov, H. M. J. Bastiaens, J. Huskens, W. G. van der Wiel, P. E. Hegeman, G. C. S. Brons, K.-J. Boller and F. Bijkerk, "Improved resolution for soft-x-ray monochromatization using lamellar multilayer gratings," in Advances in X-Ray/EUV Optics and Components VI, vol. 8139 of Proc. of SPIE, pp. 81390Q-1/8, 2011.

[75] H. V. Jansen, M. J. de Boer, S. Unnikrishnan, M. C. Louwerse and M. C. Elwenspoek, "Black silicon method X: a review on high speed and selective plasma etching of silicon with profile control: an in-depth comparison between Bosch and cryostat DRIE processes as a roadmap to next generation equipment," Journal of Micromechanics and Microengineering, vol. 19, no. 3, p. 033001, 2009.

[76] D. R. Lide, ed., CRC Handbook of Chemistry and Physics. Boca Raton: CRC Press, 84 th ed., 2003.

[77] A. L. Goodyear, S. Mackenzie, D. L. Olynick and E. H. Anderson, "High resolution inductively coupled plasma etching of $30 \mathrm{~nm}$ lines and spaces in tungsten and silicon," Journal of Vacuum Science and Technology B, vol. 18, no. 6 , pp. 3471-3475, 2000.

[78] W. Chu, K. W. Foster, L. M. Shirey, K. W. Rhee, J. Kosakowski, I. P. Isaacson, D. McCarthy, C. R. Eddy, Jr., E. A. Dobisz, C. R. K. Marrian and M. C. Peckerar, "Reactive ion etching of high-aspect-ratio $100 \mathrm{~nm}$ linewidth features in tungsten," Applied Physics Letters, vol. 64, no. 16, pp. $2172-2174,1994$.

[79] L. P. Yeo, S. L. Poh, Y. C. Lam and M. B.-E. Chan-Park, "Plasma polymerization of $\mathrm{C}_{4} \mathrm{~F}_{8}$ thin film on high aspect ratio silicon molds," in Innovation in Manufacturing Systems and Technology (IMST); SingaporeMIT Alliance (SMA), 2005. 
[80] K. Richter, M. Orfert, S. Howitz and S. Thierbach, "Deep plasma silicon etch for microfluidic applications," Surface and Coatings Technology, vol. 116-119, pp. $461-467,1999$.

[81] L. L. Balakireva and I. V. Kozhevnikov, "Two-period multilayer mirrors for the soft x-ray region," in X-Ray Optics and Surface Science, vol. 2453 of Proc. of SPIE, pp. 67-79, 1995.

[82] L. A. Woldering, R. W. Tjerkstra, H. V. Jansen, I. D. Setija and W. L. Vos, "Periodic arrays of deep nanopores made in silicon with reactive ion etching and deep UV lithography," Nanotechnology, vol. 19, no. 14, p. $145304,2008$.

[83] E. Gat, F. Bounasri, M. Chaker, M.-F. Ravet, M. Moisan and J. Margot, "Temperature effects on tungsten etching," Microelectronic Engineering, vol. 30, no. 14, pp. $337-340,1996$.

[84] R. Petri, D. Henry, J.-M. Francou, N. Sadeghi and M. Vayer-Besançon, "Tungsten etching in low-pressure $\mathrm{SF}_{6}$ plasma: Influence of the surface temperature," Journal of Applied Physics, vol. 75, no. 2, pp. 1171-1178, 1994.

[85] E. Lassner and W.-D. Schubert, Tungsten: properties, chemistry, technology of the element, alloys, and chemical compounds. Kluwer Academic/Plenum Publishers, 1999.

[86] C. C. Tang and D. W. Hess, "Tungsten Etching in $\mathrm{CF}_{4}$ and $\mathrm{SF}_{6}$ Discharges," Journal of the Electrochemical Society, vol. 131, no. 1, pp. 115$120,1984$.

[87] M. J. H. Kessels, J. Verhoeven, F. D. Tichelaar and F. Bijkerk, "Ioninduced interface layer formation in W/Si and WRe/Si multilayers," Surface Science, vol. 582, pp. 227-234, 2005.

[88] H. Fujiwara, Y. Ueda, A. Awasthi, N. Krishnamurthy and S. P. Garg, "Determination of the standard free energies of formation for tungsten silicides by EMF measurements using lithium silicate liquid electrolyte," Journal of Alloys and Compounds, vol. 391, no. 12, pp. 307 - 312, 2005.

[89] M. S. Chandrasekharaiah, J. L. Margrave and P. A. G. O'Hare, "The Disilicides of Tungsten, Molybdenum, Tantalum, Titanium, Cobalt, and Nickel, and Platinum Monosilicide: A Survey of Their Thermodynamic Properties," Journal of Physical and Chemical Reference Da, vol. 22, no. 6, pp. 1459-1468, 1993.

[90] G. S. Oehrlein and J. L. Lindstöm, "Competitive reactions of fluorine and oxygen with $\mathrm{W}, \mathrm{WSi}_{2}$, and $\mathrm{Si}$ surfaces in reactive ion etching using $\mathrm{CF}_{4} / \mathrm{O}_{2}$," Journal of Vacuum Science and Technology A, vol. 7, no. 3, pp. 1035-1041, 1989. 
[91] A. J. van Roosmalen, J. A. G. Baggerman and S. J. H. Brader, Dry etching for VLSI. Springer, 1991.

[92] Y. Mita, M. Sugiyama, M. Kubota, F. Marty, T. Bourouina and T. Shibata, "Aspect ratio dependent scalloping attenuation in DRIE and an application to low-loss fiber-optical switches," in 19th IEEE International Conference on MEMS, 2006, Istanbul., pp. 114 -117, 2006.

[93] P. Desauté, H. Merdji, W. Greiner, T. Missalla, C. Chenais-Popovics and P. Troussel, "Characterization of a high resolution transmission grating," Optics Communications, vol. 173, no. 16, pp. 37 - 43, 2000.

[94] "Institute for Physics of Microstructures of the Russian Academy of Science, 'X-Ray company'." 603600, GSP105, Nizhny Novgorod, Russia.

[95] E. Louis, A. E. Yakshin, T. Tsarfati and F. Bijkerk, "Nanometer interface and materials control for multilayer EUV-optical applications," Progress in Surface Science, vol. 86, no. 1112, pp. 255 - 294, 2011.

[96] B. Mertens, M. Weiss, H. Meiling, R. Klein, E. Louis, R. Kurt, M. Wedowski, H. Trenkler, B. Wolschrijn, R. Jansen, A. van de Runstraat, R. Moors, K. Spee, S. Plöger and R. van de Kruijs, "Progress in EUV optics lifetime expectations," Microelectronic Engineering, vol. 7374, no. 0, pp. 16 - 22, 2004. Micro and Nano Engineering 2003.

[97] N. Benoit, S. Yulin, T. Feigl and N. Kaiser, "Radiation stability of EUV Mo/Si multilayer mirrors," Physica B: Condensed Matter, vol. 357, no. 12, pp. $222-226,2005$.

[98] H. Meiling, B. Mertens, F. Stietz, M. Wedowski, R. Klein, R. Kurt, E. Louis and A. E. YAKSHIN, "Prevention of Mo/Si multilayer reflection loss in EUVL tools," in Soft X-Ray and EUV Imaging Systems II, vol. 4506 of Proc. of SPIE, pp. 93-104, 2001.

[99] C. Tarrio and S. Grantham, "Synchrotron beamline for extremeultraviolet multilayer mirror endurance testing," Review of Scientific Instruments, vol. 76, no. 5, p. 056101, 2005.

[100] S. Bajt, H. N. Chapman, N. Nguyen, J. Alameda, J. C. Robinson, M. Malinowski, E. Gullikson, A. Aquila, C. Tarrio and S. Grantham, "Design and performance of capping layers for Extreme-UltraViolet multilayer mirrors," Applied Optics, vol. 42, no. 28, pp. 5750-5758, 2003.

[101] K. Boller, R. P. Haelbich, H. Hogrefe, W. Jark and C. Kunz, "Investigation of carbon contamination of mirror surfaces exposed to synchrotron radiation," Nuclear Instruments and Methods in Physics Research, vol. 208, no. 13, pp. 273 - 279, 1983. 
[102] D. G. Stearns, M. B. Stearns, Y. Cheng, J. H. Stith and N. M. Ceglio, "Thermally induced structural modification of Mo/Si multilayers," Journal of Applied Physics, vol. 67, no. 5, pp. 2415-2427, 1990.

[103] T. Böttger, D. C. Meyer, P. Paufler, S. Braun, M. Moss, H. Mai and E. Beyer, "Thermal stability of Mo/Si multilayers with boron carbide interlayers," Thin Solid Films, vol. 444, no. 12, pp. 165 - 173, 2003.

[104] J. Bosgra, J. Verhoeven, R. W. E. van de Kruijs, A. E. Yakshin and F. Bijkerk, "Non-constant diffusion characteristics of nanoscopic Mo/Si interlayer growth," Thin Solid Films, vol. 522, pp. 228-232, 2012.

[105] R. W. E. van de Kruijs, S. Bruijn, A. Yakshin, I. Nedelcu and F. Bijkerk, "Interface diffusion kinetics and lifetime scaling in multilayer Bragg optics," in Advances in X-Ray/EUV Optics and Components VI, vol. 8139 of Proc. of SPIE, pp. 81390A-1/9, 2011.

[106] I. Nedelcu, R. W. E. van de Kruijs, A. E. Yakshin and F. Bijkerk, "Thermally enhanced interdiffusion in Mo/Si multilayers," Journal of Applied Physics, vol. 103, no. 8, p. 083549, 2008.

[107] C. D. Wagner, A. V. Naumkin, A. Kraut-Vass, J. W. Allison, C. J. Powell and J. R. Rumble, Jr., "NIST X-ray Photoelectron Spectroscopy Database 20, Version 3.5 (Web version)," 2003.

[108] G. Beamson and D. Briggs, High Resolution XPS of Organic Polymers: The Scienta ESCA300 Database. John Wiley \& Sons, Chichester, 1992.

[109] E. Z. Kurmaev, S. N. Shamin, V. R. Galakhov, G. Wiech, E. Majkova and S. Luby, "Characterization of W/Si multilayers by ultrasoft x-ray emission spectroscopy," Journal of Materials Research, vol. 10, pp. 907$911,1995$.

[110] D. R. Lide, CRC Handbook of Chemistry and Physics - Thermodynamic data. CRC Press: Boca Raton, FL., 89 ${ }^{\text {th }}$ ed., 2007.

[111] J. F. Moulder, W. F. Stickle, P. E. Sobol and K. D. Bomben, Handbook of X-ray Photoelectron Spectroscopy. Perkin-Elmer Corp., Eden Prairie, MN, USA, 1992.

[112] M. Singh and J. J. M. Braat, "Capping layers for extreme-ultraviolet multilayer interference coatings," Optics Letters, vol. 26, pp. 259-261, 2001.

[113] E. Zoethout, "Growth of silicon nitride thin films at room temperature." Presented at the SURF XPS/AES day, March $21^{\text {st }} 2012$, Free University of Brussel, Belgium. 


\section{Dankwoord/Acknowledgments}

Dit boekje mag dan 'mijn' proefschrift heten, het werk is zeker niet alleen door mij gedaan. Ik ben dan ook heel veel mensen dankbaar die mij de afgelopen jaren hebben geholpen.

Als eerste uiteraard Fred (Bijkerk) en Klaus die mij als promovendus hebben aangesteld en mij de afgelopen jaren wetenschappelijk ondersteund en begeleid hebben. Van mijn dagelijks begeleider Bert heb ik ontzettend veel geleerd en vele goede discussies mee gehad. De mensen van PANalytical, in het bijzonder Petra, Christian en Eugene, hebben dit project mogelijk gemaakt door onder andere het regelen van multilaag spiegels en het organiseren van metingen. De trip naar Berlijn was erg gezellig! Ook Wilfred en Jurriaan hebben als medeaanvragers een belangrijke rol gespeeld in dit project. Serkan is working hard on the Surface Acoustic Waves part of the project I also worked on and we spent several fun and interesting cleanroom hours working together. A huge contribution to this thesis and the mastermind behind single-order operation was Igor (Kozhevnikov) to whom I am very grateful!

I've had a great deal of support from the questions and discussion with the members of both the LPNO group at the University of Twente and the nSIgroup of FOM institute DIFFER (formerly Rijnhuizen). Many thanks to my former and fellow (PhD-)students and colleagues Thomas, Ruud, Kasper, Jörn, Mark, Rolf, Muharrem, Jonathan, Joan, Jean, Yin, Peter, Fred (van Goor), Carin, Rob, Kai, Wiebke, Bob, Marc, Jelle, Feng, Lantian, Marcel, Willem, Chris, Ab, Gerard, Piet, Simone, Eric, Igor (Makhotkin) and Erwin. Robbert and Andrey helped me with hard x-ray measurements and understanding the material physics behind multilayer mirrors. I also really enjoyed working with Toine, Slava and Quishi on other applications of fabricating structures in multilayer mirrors. All of you made my working days fun and interesting!

Een belangrijk deel van dit werk is uitgevoerd in het Nanolab van het $\mathrm{MESA}^{+}$Instituut voor Nanotechnologie, waar ik de volledige staf dankbaar ben voor het operationeel houden van alle apparaten en het assisteren bij het gebruik ervan. Gerard, Rico en zeker ook Mark hebben regelmatig samples voor of met mij geanalyseerd. Big thanks also to Balachander and Boris who helped me with fabrication. Zonder Meint, Henri en Regina zou de ontwikkeling van het fabricageproces ook zeker een stuk langer geduurd hebben. Important soft x-ray reflectance measurements were performed by the PTB staff, for which I am very grateful! 
Tijdens mijn promotie heb ik ook een lerarenopleiding gevolgd. Hier heb ik veel van geleerd, waaronder ook zaken die ik tijdens mijn promotie nodig had zoals presenteren en uitleggen. Door als promovendus voor de klas te hebben gestaan hoop ik toch ook leerlingen geïnspireerd te hebben bèta-studies te kiezen en misschien wel uiteindelijk zelf te gaan promoveren. Tijdens deze opleiding ben ik begeleid en heb ik veel geleerd van de mensen van de natuurkunde afdelingen van het OSG Erasmus in Oldenzaal en het Carmel college vestiging Lyceumstraat in Oldenzaal als ook Jan en Henk van ELAN.

Door mijn promotie heb ik mij de afgelopen jaren ook als persoon verder ontwikkeld en dit heeft ook zijn weerslag gehad op mijn privé leven. Andersom geldt dit zeker ook en hiervoor ben ik verschillende mensen van buiten mijn promotie om dankbaar. Dit geldt onder andere voor iedereen die betrokken is bij de SIGMA Twente, de coördinatoren groep Eerste Hulp van de Nijmeegse4-daagse en mijn (technische) duik buddy's. Verder zou ik ook mijn vrienden willen bedanken voor de bezoekjes aan de Zwarte Cross, concerten, motortochten en nog veel meer leuke dag- en weekendtripjes van de afgelopen jaren.

Mijn ouders en broertjes waren voor mij zeer belangrijk en een goede steun. Bedankt Bert-Jan en Erik dat jullie toch mijn paranimfen wilden zijn ook al wisten jullie totaal niet wat dat eigenlijk inhield. Tot slot natuurlijk een groot "dankjewel schat!" aan Carla, bij wie ik regelmatig even wat frustraties kwijt kon en precies wist wanneer ik even met rust gelaten, afgeremd of gesteund moest worden.

Bij deze een super bedankt aan iedereen, ook diegenen die ik niet bij naam heb genoemd, dat jullie het mij mogelijk hebben gemaakt deze vier jaar zo mooi af te mogen ronden!

Komt goed!

Robert 